\title{
CONSENSUS CLUSTERING FOR BAYESIAN MIXTURE MODELS
}

\author{
Stephen Coleman ${ }^{1, *}$ \\ stephen.coleman@mrc-bsu.cam.ac.uk
}

Paul D.W. Kirk ${ }^{1,2, \dagger}$

paul.kirk@mrc-bsu.cam.ac.uk

Chris Wallace $^{1,2, \dagger}$

cew540cam.ac.uk

${ }^{1}$ MRC Biostatistics Unit

${ }^{2}$ Cambridge Institute of Therapeutic Immunology \& Infectious Disease

University of Cambridge, U.K.

* Corresponding author.

$\dagger$ These authors provided an equal contribution.

March 12, 2021 


\begin{abstract}
Cluster analysis is an integral part of precision medicine and systems biology, used to define groups of patients or biomolecules. However, problems such as choosing the number of clusters and issues with high dimensional data arise consistently. An ensemble approach, such as consensus clustering, can overcome some of the difficulties associated with high dimensional data, frequently exploring more relevant clustering solutions than individual models. Another tool for cluster analysis, Bayesian mixture modelling, has alternative advantages, including the ability to infer the number of clusters present and extensibility. However, inference of these models is often performed using Markov-chain Monte Carlo (MCMC) methods which can suffer from problems such as poor exploration of the posterior distribution and long runtimes. This makes applying Bayesian mixture models and their extensions to 'omics data challenging. We apply consensus clustering to Bayesian mixture models to address these problems.
\end{abstract}

Consensus clustering of Bayesian mixture models successfully finds the generating structure across our simulation studies and captures multiple modes in the likelihood surface. This approach also offers significant reductions in runtime compared to traditional Bayesian inference when a parallel environment is available. We propose a heuristic to decide upon ensemble size and then apply consensus clustering to Bayesian integrative clustering method, showing consensus clustering can be applied to any MCMC-based clustering method. We perform an integrative analysis of three 'omics datasets for budding yeast and find clusters of co-expressed genes with shared regulatory proteins. We validate these clusters using data external to the analysis. These clusters can help assign likely function to understudied genes, for example GAS3 clusters with histones active in S-phase, suggesting a role in DNA replication.

Consensus clustering enables use of existing implementations of MCMC-based clustering methods on high-dimensional datasets, performing meaningful, reproducible inference where traditional approaches fail and faster inference where they do not. This enables researchers to use state-of-the-art Bayesian clustering methods on modern 'omics datasets, methods that can jointly model multiple datasets and can infer the number of clusters present. 
From defining a taxonomy of disease to creating molecular sets, grouping items can help us to understand and make decisions using complex biological data. For example, grouping patients based upon disease characteristics and personal omics data may allow the identification of more homogeneous subgroups, enabling stratified medicine approaches. In systems biology, defining and studying molecular sets can improve our understanding of biological systems as these sets are more interpretable than their constituent members (Hejblum et al. 2015), and study of their interactions and perturbations may have ramifications for diagnosis and drug targets (Bai et al., 2013; Emmert-Streib et al., 2014). The act of identifying such groups is referred to as "cluster analysis", and has been traditional been done using tools such as $K$-means clustering (Lloyd, 1982, Forgy, 1965) or hierarchical clustering. However, these methods have various problems. For example, in $K$-means clustering, its sensitivity to initialisation means multiple runs are required, with that which minimises some metric such as the within-cluster sum of squared errors (SSE) used (Arthur and Vassilvitskii, 2006). This problem arises as the algorithm has no guarantees on finding the global minimum of SSE. Another common problem is that traditional methods offer no measure of the uncertainty in the final clustering, a quantity of interest in many analyses. Returning to the stratified medicine example of clustering patients, there might be individuals with almost equal probability of being allocated between several clusters which might influence decisions made; however if only a point estimate is obtained this information is not available to the decision-maker. Ensemble methods offer a solution to this problem as well as reducing sensitivity to initialisation. These approaches have had great success in supervised learning, most famously in the form of Random Forest (Breiman, 2001) and boosting (Friedman, 2002). In clustering, consensus clustering (Monti et al., 2003) is a popular ensemble method which has been implemented in R (Wilkerson et al. 2010) and applied to a variety of methods (John et al., 2020; Gu et al., 2020) and problems such as cancer subtyping (Lehmann et al., 2011; Verhaak et al., 2010) and identifying subclones in single cell analysis (Kiselev et al., 2017). Consensus clustering uses $W$ runs of some base model or learner (such as $K$-means clustering) and compiles the $W$ proposed partitions into a consensus matrix, the $(i, j)^{t h}$ entries of which contain the proportion of model runs for which the $i^{t h}$ and $j^{\text {th }}$ individuals co-cluster (for this and other definitions see section 1 of Additional file 1). This proportion represents some measure of confidence in the co-clustering of any pair of items. Furthermore, ensembles can offer reductions in computational runtime. This is as the individual learners can be weaker (and thus use either less of the available data or stop before full convergence) and because the learners in most ensemble methods are independent of each other and thus enable use of a parallel environment for each of the quicker model runs (Ghaemi et al., 2009). 
Traditional clustering methods condition upon a fixed choice of $K$, the number of clusters. Choosing $K$ is a difficult problem that haunts many analyses with researchers often relying on rules of thumb to decide upon a final model choice. For example, different choices of $K$ are compared under some metric such as silhouette or SSE as a function of $K$. Monti et al. (2003) proposed some methods for choosing $K$ using the consensus matrix, but this means that any of the uncertainty about $K$ is not represented in the final clustering and each model run uses the same, fixed, number of clusters. An alternative clustering approach, model-based clustering or mixture models, embeds the cluster analysis within a formal, statistical framework (Fraley and Raftery, 2002). This means that models can be compared formally, and problems such as the choice of $K$ can be addressed as a model selection problem with all the associated tools. Mixture models are also attractive, as they have great flexibility in the type of data they can be applied to due to different choice of densities. Bayesian mixture models can directly infer $K$, treating this as another random variable that is inferred from the data. This means that the final clustering is not conditional upon a user chosen value, but $K$ is jointly modelled along with the clustering. Such inference can be performed through use of a Dirichlet Process (Ferguson, 1973), a mixture of finite mixture models (Richardson and Green, 1997; Miller and Harrison, 2018) or an over-fitted mixture model (Rousseau and Mengersen, 2011). These models and their extensions have a history of successful application to a diverse range of biological problems such as finding clusters of gene expression profiles (Medvedovic and Sivaganesan, 2002), cell types in flow cytometry (Chan et al., 2008; Hejblum et al., 2019) or scRNAseq experiments (Prabhakaran et al., 2016), and estimating protein localisation (Crook et al., 2018). Bayesian mixture models can be extended to jointly model the clustering across multiple datasets (Kirk et al., 2012; Gabasova et al., 2017) (section 2 of Additional file 1).

However, performing inference of Bayesian mixture models is a difficult task. Variational inference (Blei et al., 2006) (VI) may be used to perform approximate inference (Martin et al., 2020), but, while VI is powerful, it can struggle with multi-modality, underestimates the variance in the posterior distribution (Turner and Sahani, 2011) and it has been shown to have a very computationally heavy initialisation cost to have good results (Wang and Dunson, 2011). Implementation is difficult, requiring either complex derivations (see the Appendix Supplementary Methods of Argelaguet et al. (2018) for an example) or black-box, approximate solutions (Kucukelbir et al., 2017). Markov chain Monte Carlo (MCMC) methods are the most common tool for performing Bayesian inference. In Bayesian clustering methods, they are used to construct a chain of clusterings and an assessment of the convergence of this chain is made to determine if its behaviour aligns with the expected asymptotic theory. However, in practice individual chains often fail to explore the full support of the posterior distribution despite the ergodicity of MCMC methods (see, e.g., the Supplementary Materials of Strauss et al. (2020)) and can experience long runtimes. Some MCMC methods make efforts to 
overcome the problem of exploration, often at the cost of increased computational cost per iteration. See, e.g., (Robert et al., 2018; Yao et al., 2020; Bouchard-Côté et al., 2017) for examples of problems and attempted solutions for MCMC methods.

We propose that applying consensus clustering to Bayesian mixture models can overcome some of the issues endemic in high dimensional Bayesian clustering. (Monti et al., 2003) suggest this application as part of their original paper, but no investigation has been attempted to our knowledge. This ensemble approach sidesteps the problems of convergence associated MCMC methods and offers computational gains through using shorter chains run in parallel. Furthermore, this approach could be directly used on any existing MCMC based implementation of Bayesian mixture models or their extensions and would avoid the re-implementation process that changing to newer MCMC methods or VI would entail.

We propose a heuristic for deciding upon the ensemble width (the number of learners used, $W$ ) and the ensemble depth (the number of iterations run within each chain, $D$ ), inspired by the use of scree plots in Principal Component Analysis (PCA, Wold et al., 1987).

We show via simulation that ensembles consisting of short chains can be sufficient to successfully recover generating structure. We also show that consensus clustering explores as many or more modes of the likelihood surface than either standard Bayesian inference or Mclust, a maximum likelihood method, all while offering improvements in runtime to traditional Bayesian inference.

We use consensus clustering of Multiple Dataset Interation (MDI), a Bayesian integrative clustering method, to analyse multiple 'omics datasets relating to the cell cycle of Saccharomyces cerevisiae to show that consensus clustering can applied to more complex MCMC-based clustering methods and real datasets.

\section{Methods}

\section{Consensus clustering for Bayesian mixture models}

We apply consensus clustering to MCMC based Bayesian clustering models using the method described in algorithm 11. Our application of consensus clustering has two main parameters at the ensemble level, the chain depth, $D$, and ensemble width, $W$. We infer a point clustering from the consensus matrix using the maxpear function (Fritsch et al. 2009) from the R package mcclust (Fritsch, 2012) to (section 3 of Additional file 1 for details). 
Data: $X=\left(x_{1}, \ldots, x_{N}\right)$

Input:

The number of chains to run, $W$

The number of iterations within each chain, $D$

A clustering method that uses MCMC methods to generate samples of clusterings of the data

Cluster $(X, d)$

\section{Output:}

A predicted clustering, $\hat{Y}$

The consensus matrix $\mathbf{M}$

begin

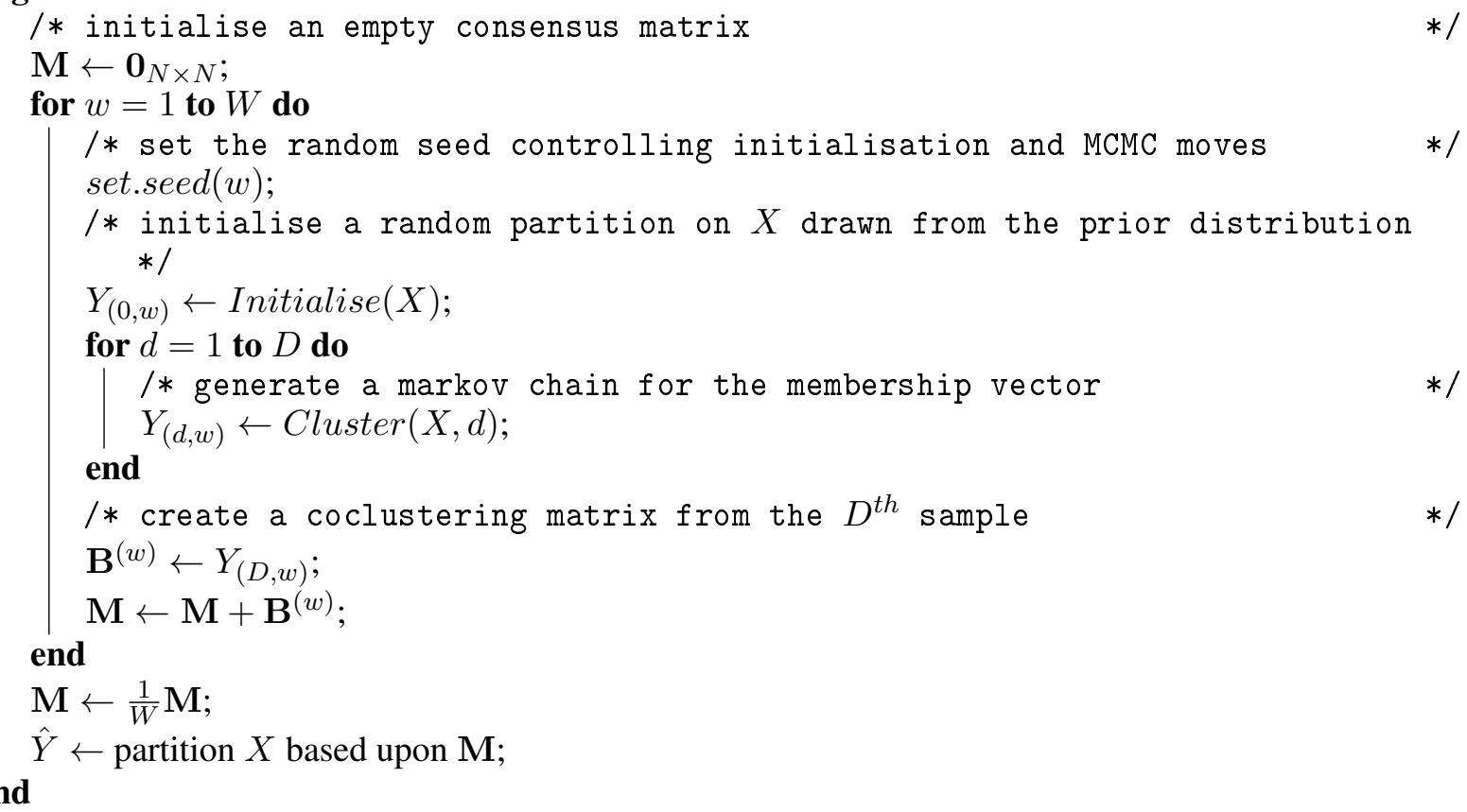

Algorithm 1: Consensus clustering for Bayesian mixture models.

\section{Determining the ensemble depth and width}

As our ensemble sidesteps the problem of convergence within each chain, we need an alternative stopping rule for growing the ensemble in chain depth, $D$, and number of chains, $W$. We propose a heuristic based upon the consensus matrix to decide if a given value of $D$ and $W$ are sufficient. We suspect that increasing $W$ and $D$ might continuously improve the performance of the ensemble, but we observe in our simulations that these improvements will become smaller and smaller for greater values, approaching some asymptote for each of $W$ and $D$. We notice that this behaviour is analogous to PCA in that where for consensus clustering some improvement might always be expected for increasing chain depth or ensemble width, more variance will always be captured by increasing the number of components used in PCA. However, increasing this number beyond some threshold has diminishing returns, diagnosed in PCA by a scree plot. Following from this, we recommend, for some set of ensemble parameters, $D^{\prime}=\left\{d_{1}, \ldots, d_{I}\right\}$ and $W^{\prime}=\left\{w_{1}, \ldots, w_{J}\right\}$, find the mean absolute difference of the consensus matrix for the $d_{i}^{\text {th }}$ iteration from $w_{j}$ chains to that for 
the $d_{(i-1)}^{\text {th }}$ iteration from $w_{j}$ chains and plot these values as a function of chain depth, and the analogue for sequential consensus matrices for increasing ensemble width and constant depth.

If this heuristic is used, we believe that the consensus matrix and the resulting inference should be stable (see, e.g., Von Luxburg and Ben-David, 2005; Meinshausen and Bühlmann, 2010), providing a robust estimate of the clustering. In contrast, if there is still strong variation in the consensus matrix for varying chain length or number, then we believe that the inferred clustering is influenced significantly by the random initialisation and that the inferred partition is unlikely to be stable for similar datasets or reproducible for a random choice of seeds.

\section{Simulation study}

We use a finite mixture with independent features as the data generating model within the simulation study. We include "irrelevant features" (Law et al., 2003) that have global parameters rather than cluster specific parameters and use the generating model:

$$
\begin{aligned}
& p(X, c, \theta, \pi \mid K) \\
& \quad=p(K) p(\pi \mid K) p(\theta \mid K) \prod_{i=1}^{N} p\left(c_{i} \mid \pi, K\right) \prod_{p=1}^{P} p\left(x_{i p} \mid c_{i}, \theta_{c_{i} p}\right)^{\phi_{p}} p\left(x_{i p} \mid \theta_{p}\right)^{\left(1-\phi_{p}\right)}
\end{aligned}
$$

for data $X=\left(x_{1}, \ldots, x_{N}\right)$, cluster label or allocation variable $c=\left(c_{1}, \ldots, c_{N}\right)$, cluster weight $\pi=$ $\left(\pi_{1}, \ldots, \pi_{K}\right), K$ clusters and the relevance variable, $\phi \in\{0,1\}$ with $\phi_{p}=1$ indicating that the $p^{t h}$ feature is relevant to the clustering. We used a Gaussian density, so $\theta_{k p}=\left(\mu_{k p}, \sigma_{k p}^{2}\right)$. We defined three scenarios and simulated 100 datasets in each (Figure 1 and Table 1) Additional details of the simulation process and additional scenarios are included in section 4.1 of Additional file $1 \mathrm{~s}$.

Table 1: Parameters defining the simulation scenarios as used in generating data and labels. $\Delta \mu$ is the distance between neighbouring cluster means within a single feature. The number of relevant features $\left(P_{s}\right)$ is $\sum_{p} \phi_{p}$, and $P_{n}=P-P_{s}$.

\begin{tabular}{l|ccccccc}
\hline Scenario & $N$ & $P_{s}$ & $P_{n}$ & $K$ & $\Delta \mu$ & $\sigma^{2}$ & $\pi$ \\
\hline 2D & 100 & 2 & 0 & 5 & 3.0 & 1 & $\left(\frac{1}{5}, \frac{1}{5}, \frac{1}{5}, \frac{1}{5}, \frac{1}{5}\right)$ \\
Small N, large P & 50 & 500 & 0 & 5 & 1.0 & 1 & $\left(\frac{1}{5}, \frac{1}{5}, \frac{1}{5}, \frac{1}{5}, \frac{1}{5}\right)$ \\
Irrelevant features & 200 & 20 & 100 & 5 & 1.0 & 1 & $\left(\frac{1}{5}, \frac{1}{5}, \frac{1}{5}, \frac{1}{5}, \frac{1}{5}\right)$ \\
\hline
\end{tabular}

In each of these scenarios we apply a variety of methods (listed below) and compare the inferred point clusterings to the generating labels using the Adjusted Rand Index (Hubert and Arabie, 1985) (ARI). 


\section{Consensus clustering for Bayesian mixture models}

2D

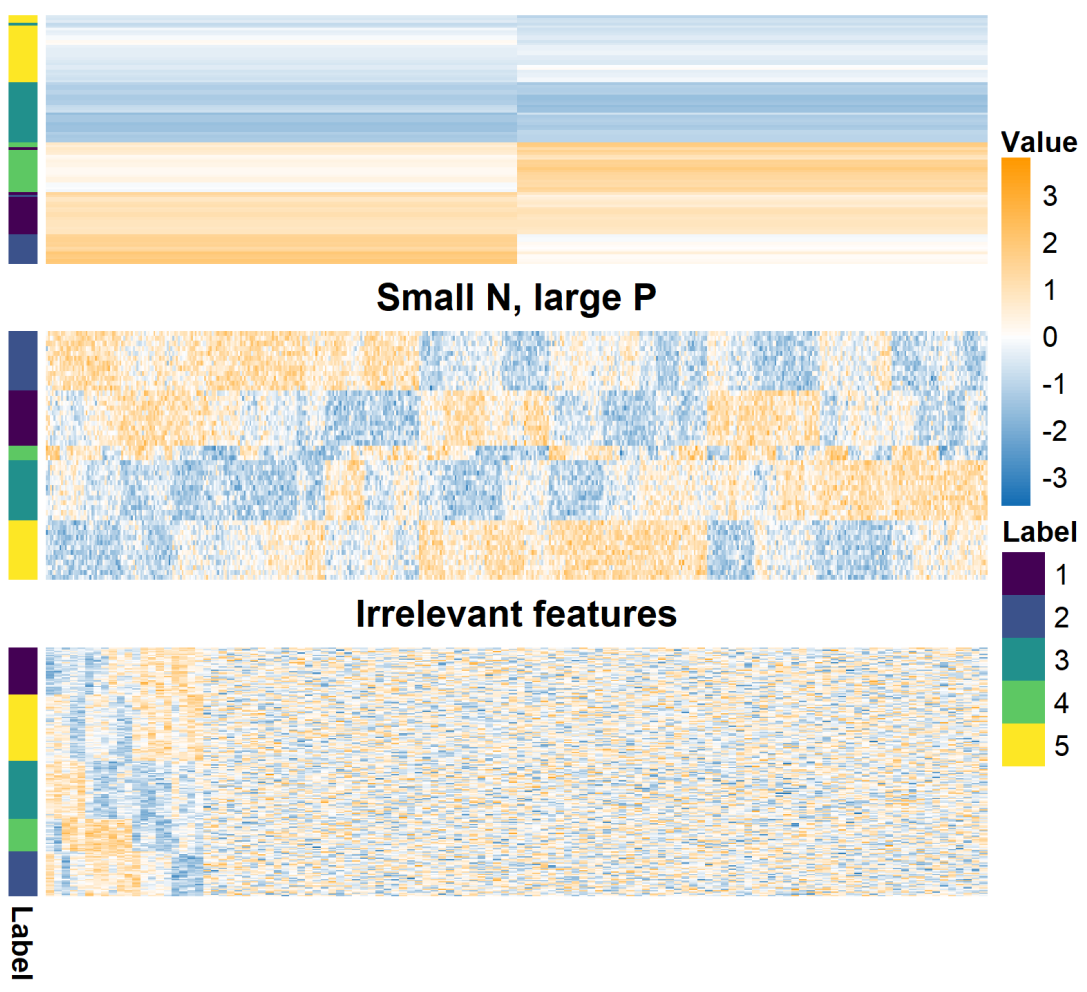

Figure 1: Example of generated datasets. Each row is an item being clustered and each column a feature of generated data. The rows and relevant features of each dataset are ordered by hierarchical clustering. For the irrelevant features dataset, only the 20 relevant features are used to order rows. The 2D dataset should enable proper mixing of chains in the MCMC. The small $N$, large $P$ case has clear structure (observable by eye). This is intended to highlight the problems of poor mixing due to high dimensions even when the generating labels are quite identifiable. In the irrelevant features case, the structure is clear in the relevant features (on the left-hand side of this heatmap). This setting is intended to test how sensitive each approach is to noise.

- Mclust, a maximum likelihood implementation of finite mixture models (for a range of modelled clusters, $K)$,

- 10 chains of 1 million iterations, thinning to every thousandth sample for the overfitted Bayesian mixture model, and

- A variety of consensus clustering ensembles defined by inputs of $W$ chains and $D$ iterations within each chain (see algorithm 1 ) with $W \in\{1,10,30,50,100\}$ and $D \in\{1,10,100,1000,10000\}$.

The ARI is a measure of similarity between two partitions, $c_{1}, c_{2}$, corrected for chance, with 0 indicating $c_{1}$ is no more similar to $c_{2}$ than a random partition would be expected to be and a value of 1 showing that $c_{1}$ and $c_{2}$ perfectly align. Details of the methods in the simulation study can be found in sections $4.2,4.3$ and 4.4 of Additional file 1. 
Mclust

Mclust (Scrucca et al., 2016) is a function from the R package mclust. It estimates Gaussian mixture models for $K$ clusters based upon the maximum likelihood estimator of the parameters. it initialises upon a hierarchical clustering of the data cut to $K$ clusters. A range of choices of $K$ and different covariance structures are compared and the "best" selected using the Bayesian information criterion, (Schwarz et al., 1978) (details in section 4.2 of Additional file 1).

\section{Bayesian inference}

To assess within-chain convergence of our Bayesian inference we use the Geweke $Z$-score statistic (Geweke et al. 1991). Of the chains that appear to behave properly we then asses across-chain convergence using $\hat{R}$ (Gelman et al., 1992) and the recent extension provided by (Vats and Knudson, 2018). If a chain has reached its stationary distribution the Geweke $Z$-score statistic is expected to be normally distributed. Normality is tested for using a Shapiro-Wilks test (Shapiro and Wilk, 1965). If a chain fails this test (i.e., the associated $p$-value is less than 0.05), we assume that it has not achieved stationarity and it is excluded from the remainder of the analysis. The samples from the remaining chains are then pooled and a posterior similarity matrix (PSM) constructed. We use the maxpear function to infer a point clustering. For more details see section 4.3 of Additional file 1.

\section{Analysis of the cell cycle in budding yeast}

\section{Datasets}

The cell cycle is crucial to biological growth, repair, reproduction, and development (Tyson et al., 2013, Chen et al., 2004; Alberts et al., 2002) and is highly conserved among eukaryotes (Alberts et al., 2002). This means that understanding of the cell cycle of $S$. cerevisiae can provide insight into a variety of cell cycle perturbations including those that occur in human cancer (Ingalls et al., 2007; Chen et al., 2004) and ageing (Jiménez et al., 2015). We aim to create clusters of genes that are co-expressed in the cell cycle, have common regulatory proteins and share a biological function. To achieve this, we use three datasets that were generated using different 'omics technologies and target different aspects of the molecular biology underpinning the cell cycle process.

- Microarray profiles of RNA expression from (Granovskaia et al., 2010), comprising measurements of cell-cycle-regulated gene expression at 5-minute intervals for 200 minutes (up to three cell division cycles) and is referred to as the time course dataset. The cells are synchronised at the START 
checkpoint in late G1-phase using alpha factor arrest (Granovskaia et al., 2010). We include only the genes identified by Granovskaia et al. (2010) as having periodic expression profiles.

- Chromatin immunoprecipitation followed by microarray hybridization (ChIP-chip) data from Harbison et al.(2004). This dataset discretizes $p$-values from tests of association between 117 DNA-binding transcriptional regulators and a set of yeast genes. Based upon a significance threshold these $p$-values are represented as either a 0 (no interaction) or a 1 (an interaction).

- Protein-protein interaction (PPI) data from BioGrid (Stark et al. 2006). This database consists of of physical and genetic interactions between gene and gene products, with interactions either observed in high throughput experiments or computationally inferred. The dataset we used contained 603 proteins as columns. An entry of 1 in the $(i, j)^{t h}$ cell indicates that the $i^{\text {th }}$ gene has a protein product that is believed to interact with the $j^{\text {th }}$ protein.

The datasets were reduced to the 551 genes with no missing data in the PPI and ChIP-chip data, as in Kirk et al. (2012).

\section{Multiple dataset integration}

We applied consensus clustering to MDI for our integrative analysis. Details of MDI are in section 2.2 of Additional file 1, but in short MDI jointly models the clustering in each dataset, inferring individual clusterings for each dataset. These partitions are informed by similar structure in the other datasets, with MDI learning this similarity as it models the partitions. The model does not assume global structure. This means that the similarity between datasets is not strongly assumed in our model; individual clusters or genes that align across datasets are based solely upon the evidence present in the data and not due to strong modelling assumptions. Thus, datasets that share less common information can be included without fearing that this will warp the final clusterings in some way.

The datasets were modelled using a mixture of Gaussian processes in the time course dataset and Multinomial distributions in the ChIP-chip and PPI datasets.

\section{Results}

\section{Simulated data}

We use the ARI between the generating labels and the inferred clustering of each method to be our metric of predictive performance. In Figure 2, we see Mclust performs very well in the 2D and Small $N$, large $P$ 


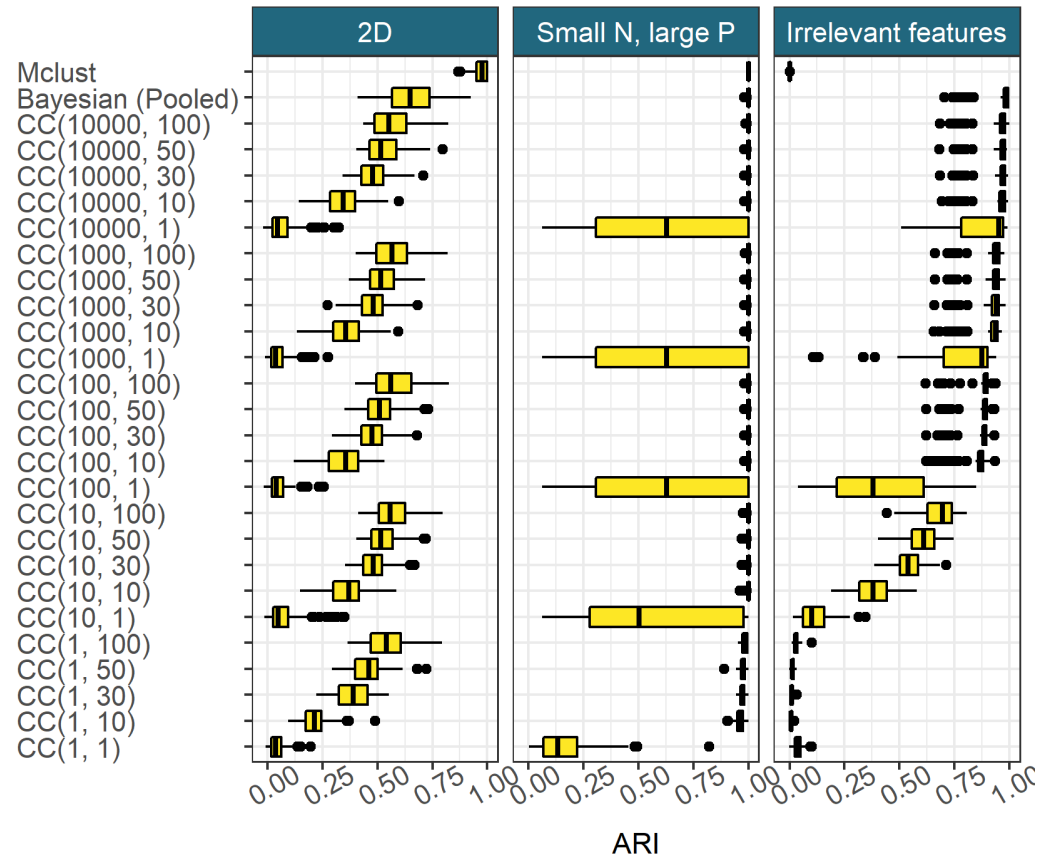

Figure 2: Model performance in the 100 simulated datasets for each scenario, defined as the ARI between the generating labels and the inferred clustering. $C C(d, w)$ denotes consensus clustering using the clustering from the $d^{\text {th }}$ iteration from $w$ different chains.

scenarios, correctly identifying the true structure However, the irrelevant features scenario sees a collapse in performance, Mclust is blinded by the irrelevant features and identifies a clustering of $K=1$.

The pooled samples from multiple long chains performs very well across all scenarios and appears to act as an upper bound on the more practical implementations of consensus clustering.

Consensus clustering does uncover some of the generating structure in the data, even using a small number of short chains. With sufficiently large ensembles and chain depth, consensus clustering is close to the pooled Bayesian samples in predictive performance. It appears that for a constant chain depth increasing the ensemble width used follows a pattern of diminishing returns. There are strong initial gains for a greater ensemble width, but the improvement decreases for each successive chain. A similar pattern emerges in increasing chain length for a constant number of chains (Figure 2).

We see very little difference between the similarity matrix from the pooled samples and the consensus clustering (Figure 3). Similar clusters emerge, and we see comparable confidence in the pairwise clusterings. For the PSMs from the individual chains, all entries are 0 or 1 . This means only a single clustering is sampled within each chain, implying very little uncertainty in the partition. However, three different modes emerge across the chains showing that the chains are failing to explore the full support of the posterior 


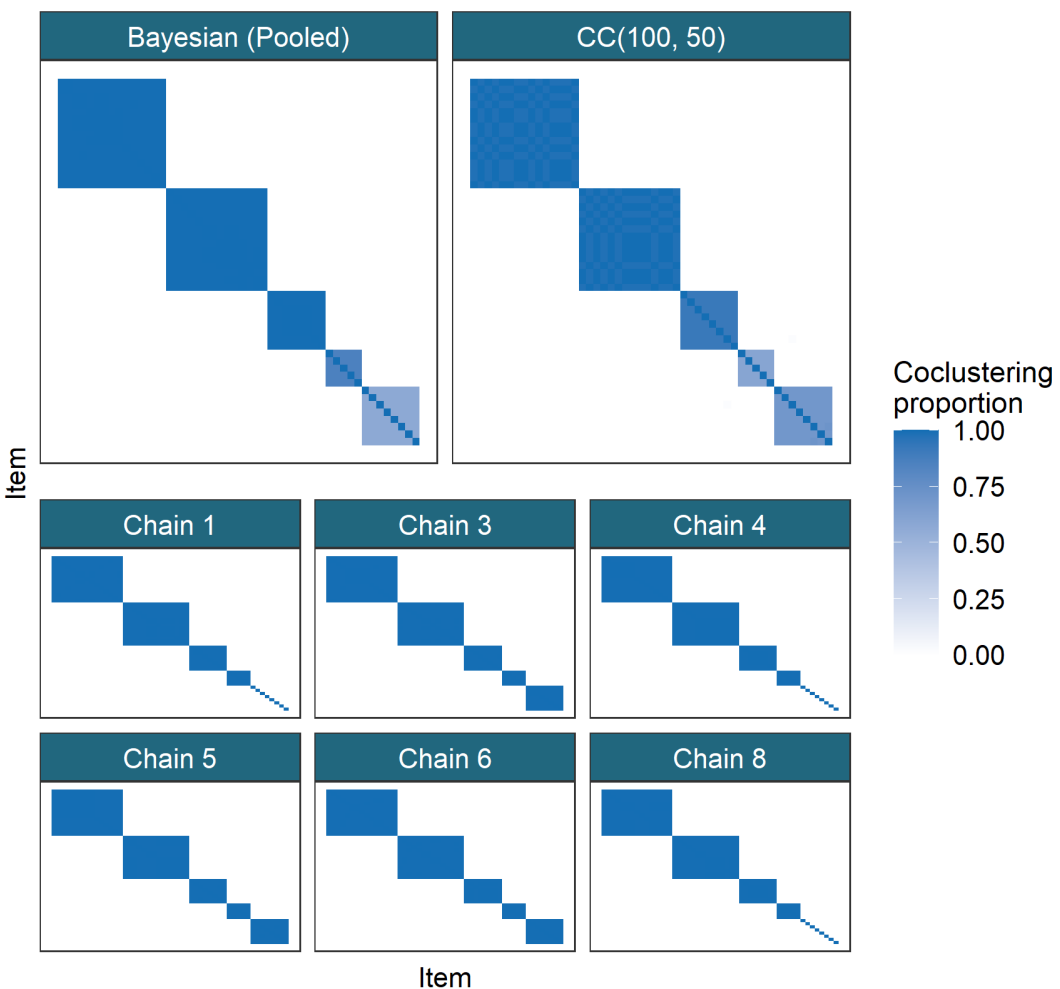

Figure 3: Comparison of similarity matrices from a dataset for the Small $N$, large $P$ scenario. In each matrix, the $(i, j)^{t h}$ entry is the proportion of clusterings for which the $i^{t h}$ and $j^{t h}$ items co-clustered for the method in question. In the first row the PSM of the pooled Bayesian samples is compared to the CM for CC $(100,50)$, with a common ordering of rows and columns in both heatmaps. In the following rows, 6 of the long chains that passed the tests of convergence are shown.

distribution of the clustering and are each unrepresentative of the uncertainty in the final clustering. This shows that consensus clustering is exploring more possible clusterings than any individual chain and, as it explores a similar space to the pooled samples which might be considered more representative of the posterior distribution than any one chain, it suggests it better describes the true uncertainty present than any single chain. It also shows that pooling chains offers robustness to multi-modality (as expected for an ensemble) and the ARI for the pooled samples is an upper bound on the performance for the individual long chains.

Figure 4 shows that chain length is directly proportional to the time taken for the chain to run. This means that using an ensemble of shorter chains, as in consensus clustering, can offer large reductions in the time cost of analysis when a parallel environment is available compared to standard Bayesian inference. Even on a laptop of 8 cores running an ensemble of 1,000 chains of length 1,000 will require approximately half as much time as running 10 chains of length 100,000 due to parallelisation, and the potential benefits are far greater when using a large computing cluster. 


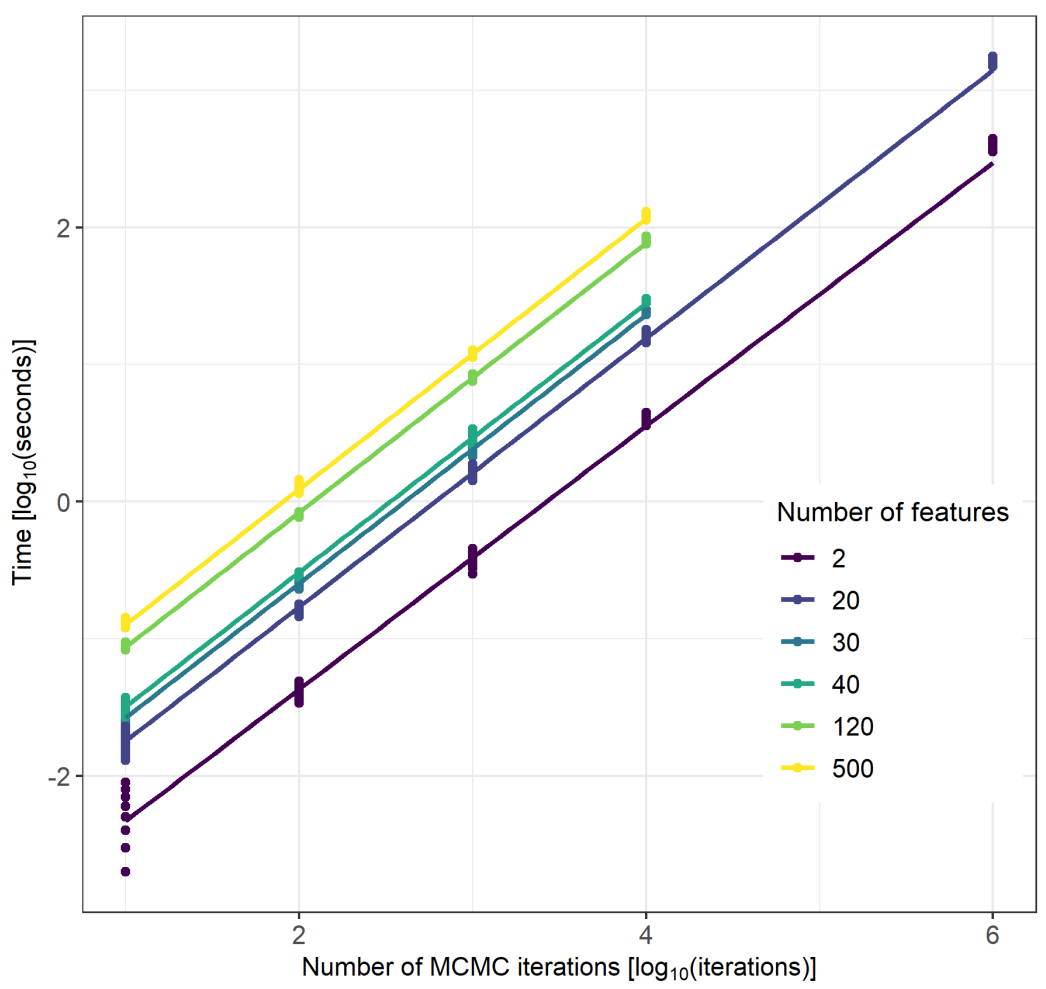

Figure 4: The time taken for different numbers of iterations of MCMC moves in $\log _{10}($ seconds $)$. The relationship between chain length, $D$, and the time taken is linear (the slope is approximately 1 on the $\log _{10}$ scale), with a change of intercept for different dimensions. The runtime of each Markov chain was recorded using the terminal command time, measured in milliseconds.

Additional results for these and other simulations are in section 4.4 of Additional file 1.

\section{Multi-omics analysis of the cell cycle in budding yeast}

We use the stopping rule proposed in to determine our ensemble depth and width. In Figure 5, we see that the change in the consensus matrices from increasing the ensemble depth and width is diminishing in keeping with results in the simulations. We see no strong improvement after $D=6,000$ and increasing the number of learners from 500 to 1,000 has small effect. We therefore use the largest ensemble available, a depth $D=10001$ and width $W=1000$, believing this ensemble is stable (additional evidence in section 5.1 of Additional file 1).

We focus upon the genes that tend to have the same cluster label across multiple datasets. More formally, we analyse the clustering structure among genes for which $\hat{P}\left(c_{n l}=c_{n m}\right)>0.5$, where $c_{n l}$ denotes the cluster label of gene $n$ in dataset $l$. In our analysis it is the signal shared across the time course and ChIP-chip datasets that is strongest, with 261 genes (nearly half of the genes present) in this pairing tending to have a 


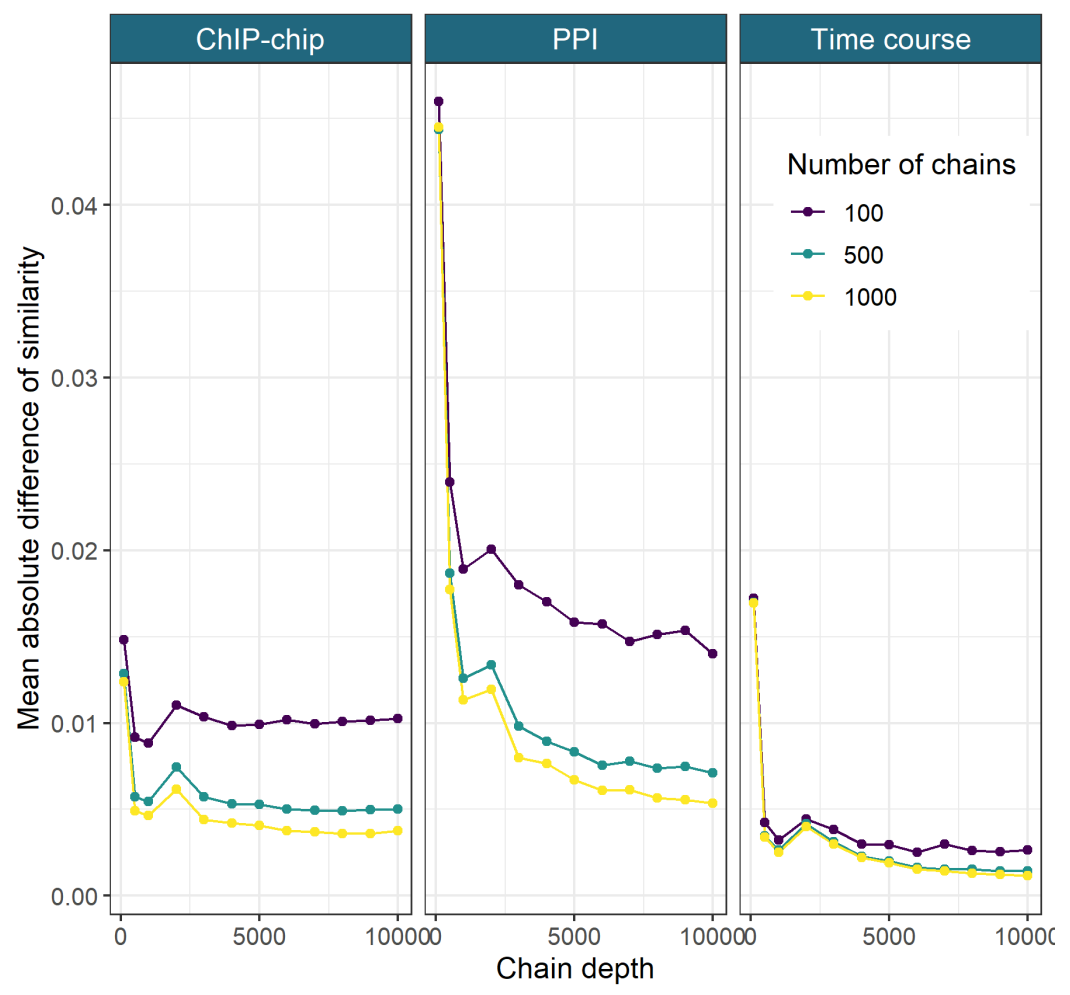

Figure 5: The mean absolute difference between the sequential Consensus matrices. For a set of chain lengths, $D^{\prime}=\left\{d_{1}, \ldots, d_{I}\right\}$ and number of chains, $W^{\prime}=\left\{w_{1}, \ldots, w_{J}\right\}$, we take the mean of the absolute difference between the consensus matrix for $\left(d_{i}, w_{j}\right)$ and $\left(d_{i-1}, w_{j}\right)$ (here $D^{\prime}=\{101,501,1001,2001, \ldots, 10001\}$ and $\left.W^{\prime}=\{100,500,1000\}\right)$.

common label, whereas only 56 genes have a common label across all three datasets. Thus, we focus upon this pairing of datasets in the results of the analysis performed using all three datasets. We show the gene expression and regulatory proteins of these genes separated by their cluster in Figure 6, In Figure 6, the clusters in the time series data have tight, unique signatures (having different periods, amplitudes, or both) and in the ChIP-chip data clusters are defined by a small number of well-studied transcription factors (TFs) Simon et al. (2001) (see Table 2 of Additional file 1).

As an example, we briefly analyse clusters 9 and 16 in greater depth. Cluster 9 has strong association with MBP1 and some interactions with SWI6, as can be seen in Figure 6. The Mbp1-Swi6p complex, MBF, is associated with DNA replication (Iyer et al., 2001). The first time point, 0 minutes, in the time course data is at the START checkpoint, or the G1/S transition. The members of cluster 9 begin highly expressed at this point before quickly dropping in expression (in the first of the 3 cell cycles). This suggests that many transcripts are produced immediately in advance of S-phase, and thus are required for the first stages of DNA synthesis. These genes' descriptions ([found using org. Sc .sgd.db, (Carlson et al., 2014), and shown in Table 3 of Additional file 1) support this hypothesis, as many of the members are associated with DNA 


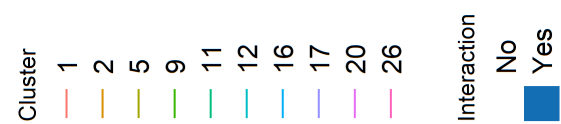
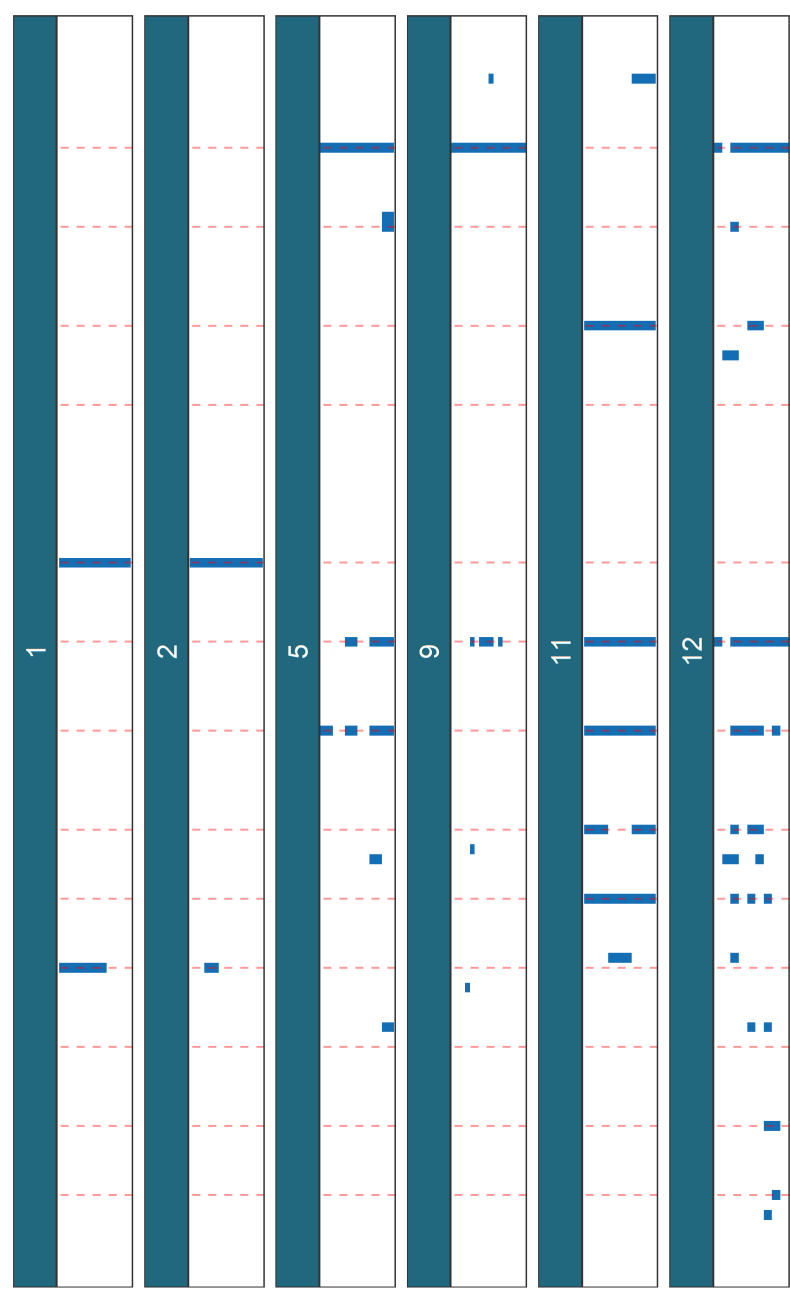

әиәऽ

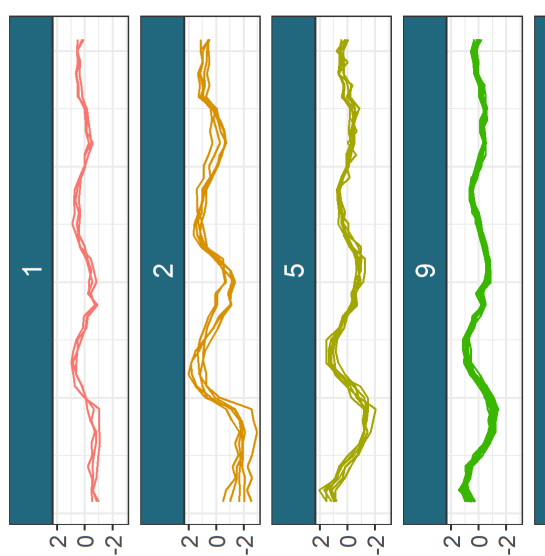

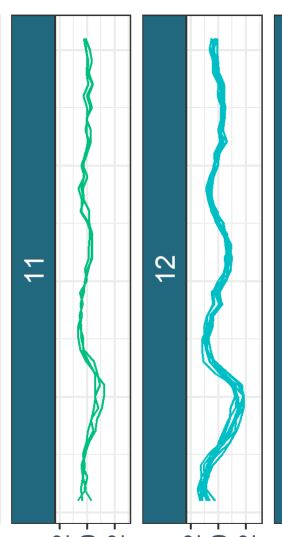
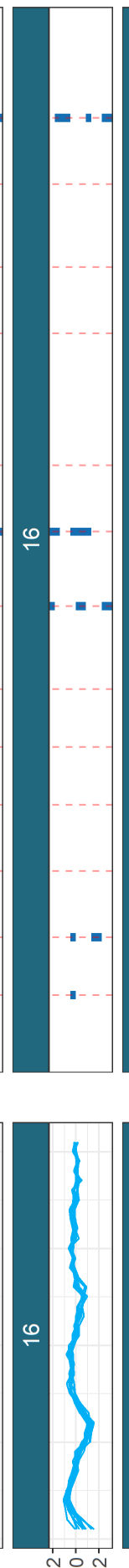
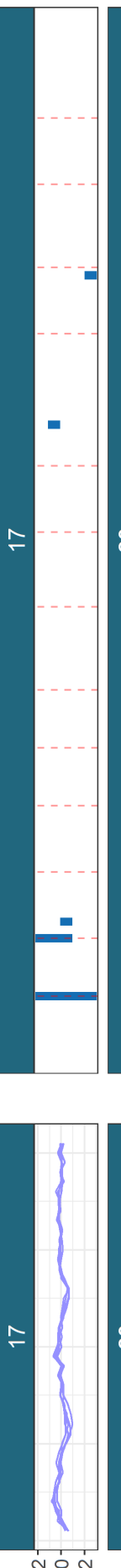
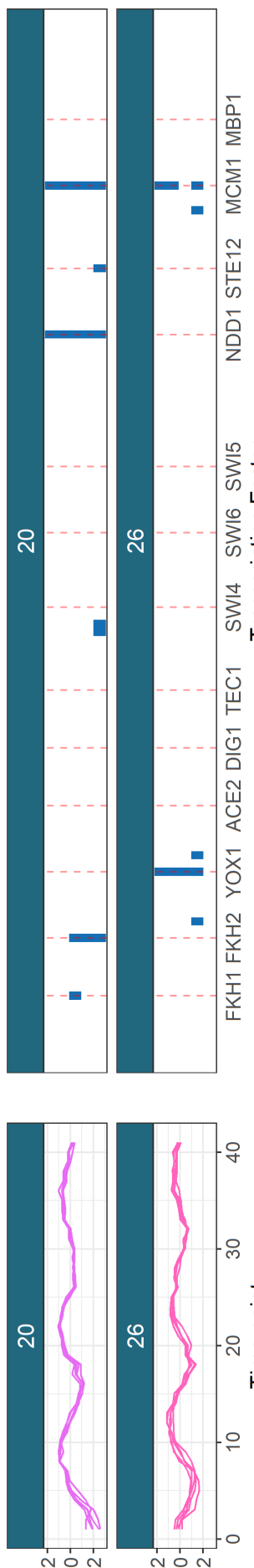

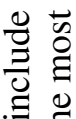

ऐ.ॅ

$\dot{0} \ddot{0}$

:

चี

赵

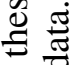

.$\Xi$

承

空

si

过

莺.

유 을

응 음

空

?

0 )

品

U

曲

르

品

ปั

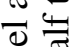

종

크

ฏ

ฮี

U

ส

츤

을

옹

Ð

둥

클

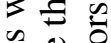

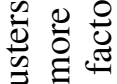

응

记

800

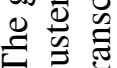

$\forall \equiv$

$\because$ ن

웡

旃文吉 
replication, repair and/or recombination. Additionally, TOF1, $M R C 1$ and $R A D 53$, members of the replication checkpoint (Bando et al., 2009; Lao et al., 2018) emerge in the cluster as do members of the cohesin complex. Cohesin is associated with sister chromatid cohesion which is established during the S-phase of the cell cycle (Tóth et al., 1999) and also contributes to transcription regulation, DNA repair, chromosome condensation, homolog pairing (Mehta et al., 2013), fitting the theme of cluster 9.

Cluster 16 appears to be a cluster of S-phase genes, consisting of GAS3, NRMI and PDS1 and the genes encoding the histones $\mathrm{H} 1, \mathrm{H} 2 \mathrm{~A}, \mathrm{H} 2 \mathrm{~B}, \mathrm{H} 3$ and $\mathrm{H} 4$. Histones are the chief protein components of chromatin (Fischle et al., 2003) and are important contributors to gene regulation (Bannister and Kouzarides, 2011). They are known to peak in expression in S-phase (Granovskaia et al. 2010), which matches the first peak of this cluster early in the time series. Of the other members, NRM1 is a transcriptional co-repressor of MBF-regulated gene expression acting at the transition from G1 to S-phase (de Bruin et al., 2006, Aligianni et al., 2009). Pds1p binds to and inhibits the Esp1 class of sister separating proteins, preventing sister chromatids separation before M-phase (Ciosk et al., 1998; Tóth et al., 1999). GAS3, is not well studied. It interacts with SMT3 which regulates chromatid cohesion, chromosome segregation and DNA replication (among other things). Chromatid cohesion ensures the faithful segregation of chromosomes in mitosis and in both meiotic divisions (Cooper et al., 2009) and is instantiated in S-phase (Tóth et al., 1999). These results, along with the very similar expression profile to the histone genes in the time course data, suggest that GAS3 may be more directly involved in DNA replication or chromatid cohesion than is currently believed.

We attempt to perform a similar analysis using traditional Bayesian inference of MDI, but after 36 hours of runtime there is no consistency or convergence across the ten chains. We use the Geweke statistic and $\hat{R}$ to reduce to the five best behaved chains (none of which appear to be converged, Additional file 1, section 5.2). If we then compare the distribution of sampled values for the $\phi$ parameters for these long chains, the final ensemble used ( $D=10001, W=1000)$ and the pooled samples from the 5 long chains, then we see that the distribution of the pooled samples from the long chains (which might be believed to sampling different parts of the posterior distribution) is closer in appearance to the distributions sampled by the consensus clustering than to any single chain (figure 7). Further disagreement between chains is shown in the Gene Ontology term over-representation analysis in section 5.3 of Additional file 1.

\section{Discussion}

Our proposed method has demonstrated good performance on simulation studies, uncovering the generating structure and approximating Bayesian inference when the Markov chain is exploring the full support of the 


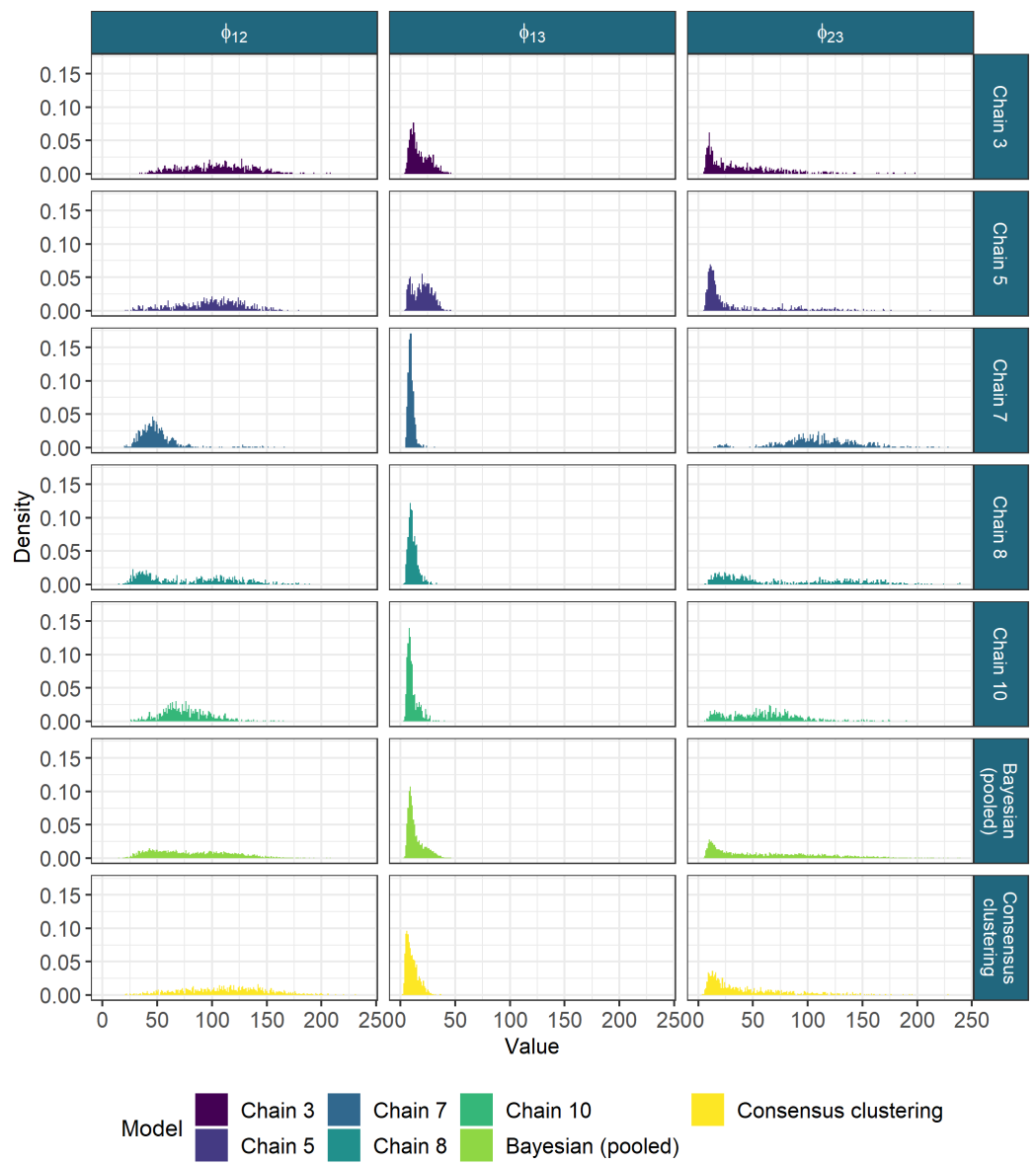

Figure 7: The sampled values for the $\phi$ parameters from the long chains, their pooled samples and the consensus using 1000 chains of depth 10,001. The long chains display a variety of behaviours. Across chains there is no clear consensus on the nature of the posterior distribution. The samples from any single chain are not particularly close to the behaviour of the pooled samples across all three parameters. It is the consensus clustering that most approaches this pooled behaviour.

posterior distribution. However, we have shown that if a finite Markov chain fails to describe the full posterior and is itself only approximating Bayesian inference, our method has better ability to represent several modes in the data than individual chains and thus offers a more consistent and reproducible analysis. Furthermore, consensus clustering is significantly faster in a parallel environment than inference using individual chains, while retaining the ability to robustly infer the number of clusters present.

We proposed a method of assessing ensemble stability and deciding upon ensemble size which we used when performing an integrative analysis of yeast cell cycle data using MDI, an extension of Bayesian mixture models that jointly models multiple datasets. We uncovered many genes with shared signal across several datasets and explored the meaning of some of the inferred clusters, using data external to the analysis. We found sensible results as well as signal for possibly novel biology. In contrast, the traditional approach to 
Bayesian inference failed here. The lack of a consistent distribution across the chains made proceeding with the Bayesian analysis difficult as choosing the result of any single chain over the others would be arbitrary and thus prone to irreproducibility. The alternative of pooling the samples, which might be considered a reasonable compromise, appears to offer a very similar solution to consensus clustering, but with longer runtime and additional steps to reduce the chains to the "best-behaved" chains. We believe that the similarity between the sampled distribution of the parameters from the pooled long chains and the consensus clustering of short chains, figure 7, suggests that sufficiently deep chains within the ensemble can be used even to perform inference of continuous variables and not only the latent clustering of the data.

Consensus clustering loses the theoretical framework of true Bayesian inference. We attempt to mitigate this with our assessment of stability in the ensemble, but this diagnosis is heuristic and subjective, and while there is empirical evidence for its success, it lacks the formal results for the tests of model convergence for Bayesian inference. Nonetheless, the results of our simulations and the multi-omics analysis show that consensus clustering can be successfully used in a broad context, being applicable to any MCMC based clustering method. It offers computational gains and improves the exploration of the clustering space, overcoming the problem of becoming trapped in specific, local extrema of the likelihood surface that emerges in high-dimensional data. This enables the application of these methods in modern 'omics datasets and, attractively, consensus clustering can be applied to existing implementations, unlike improvements to the underlying MCMC methods or alternative methods for Bayesian inference such as VI which would require re-writing software.

\section{Funding}

This work was funded by the MRC (MC UU 00002/4, MC UU 00002/13) and supported by the NIHR Cambridge Biomedical Research Centre (BRC-1215-20014). The views expressed are those of the author(s) and not necessarily those of the NHS, the NIHR or the Department of Health and Social Care. This research was funded in whole, or in part, by the Wellcome Trust [WT107881]. For the purpose of Open Access, the author has applied a CC BY public copyright licence to any Author Accepted Manuscript version arising from this submission.

\section{Availability of data and materials}

The code and datasets supporting the conclusions of this article are available in the github repository, https://github.com/stcolema/ConsensusClusteringForBayesianMixtureModels. 


\section{Authors' contributions}

SC designed the simulation study with contributions from PK and CW, performed the analyses and wrote the manuscript. PK and CW provided an equal contribution of joint supervision, directing the research and provided suggestions such as the stopping rule. All contributed to interpreting the results of the analyses. All authors revised and approved the final manuscript.

\section{References}

Bruce Alberts, Alexander Johnson, Julian Lewis, Martin Raff, Keith Roberts, and Peter Walter. The cell cycle and programmed cell death. Molecular biology of the cell, 4:983-1027, 2002.

Sofia Aligianni, Daniel H Lackner, Steffi Klier, Gabriella Rustici, Brian T Wilhelm, Samuel Marguerat, Sandra Codlin, Alvis Brazma, Robertus AM de Bruin, and Jürg Bähler. The fission yeast homeodomain protein Yox1p binds to MBF and confines MBF-dependent cell-cycle transcription to G1-S via negative feedback. PLoS Genet, 5(8):e1000626, 2009.

Ricard Argelaguet, Britta Velten, Damien Arnol, Sascha Dietrich, Thorsten Zenz, John C Marioni, Florian Buettner, Wolfgang Huber, and Oliver Stegle. Multi-omics factor analysis—a framework for unsupervised integration of multi-omics data sets. Molecular systems biology, 14(6):e8124, 2018.

David Arthur and Sergei Vassilvitskii. k-means++: The advantages of careful seeding. Technical report, Stanford, 2006.

Jane PF Bai, Alexander V Alekseyenko, Alexander Statnikov, I-Ming Wang, and Peggy H Wong. Strategic applications of gene expression: from drug discovery/development to bedside. The AAPS journal, 15(2): 427-437, 2013.

Masashige Bando, Yuki Katou, Makiko Komata, Hirokazu Tanaka, Takehiko Itoh, Takashi Sutani, and Katsuhiko Shirahige. Csm3, Tof1, and Mrc1 form a heterotrimeric mediator complex that associates with DNA replication forks. Journal of Biological Chemistry, 284(49):34355-34365, 2009.

Andrew J Bannister and Tony Kouzarides. Regulation of chromatin by histone modifications. Cell research, 21(3):381-395, 2011.

David M Blei, Michael I Jordan, et al. Variational inference for Dirichlet process mixtures. Bayesian analysis, 1(1):121-143, 2006.

Alexandre Bouchard-Côté, Arnaud Doucet, and Andrew Roth. Particle Gibbs split-merge sampling for Bayesian inference in mixture models. Journal of Machine Learning Research, 18(28), 2017. 
Leo Breiman. Random forests. Machine learning, 45(1):5-32, 2001.

M Carlson, S Falcon, H Pages, and N Li. Org. sc. sgd. db: Genome wide annotation for yeast. R package version, 2(1), 2014.

Cliburn Chan, Feng Feng, Janet Ottinger, David Foster, Mike West, and Thomas B Kepler. Statistical mixture modeling for cell subtype identification in flow cytometry. Cytometry Part A: The Journal of the International Society for Analytical Cytology, 73(8):693-701, 2008.

Katherine C Chen, Laurence Calzone, Attila Csikasz-Nagy, Frederick R Cross, Bela Novak, and John J Tyson. Integrative analysis of cell cycle control in budding yeast. Molecular biology of the cell, 15(8):3841-3862, 2004.

Rafal Ciosk, Wolfgang Zachariae, Christine Michaelis, Andrej Shevchenko, Matthias Mann, and Kim Nasmyth. An esp1/pds1 complex regulates loss of sister chromatid cohesion at the metaphase to anaphase transition in yeast. Cell, 93(6):1067-1076, 1998.

Katrina F Cooper, Michael J Mallory, Vincent Guacci, Katherine Lowe, and Randy Strich. Pds1p is required for meiotic recombination and prophase I progression in Saccharomyces cerevisiae. Genetics, 181(1): 65-79, 2009.

Oliver M Crook, Claire M Mulvey, Paul DW Kirk, Kathryn S Lilley, and Laurent Gatto. A Bayesian mixture modelling approach for spatial proteomics. PLoS computational biology, 14(11):e1006516, 2018.

Robertus AM de Bruin, Tatyana I Kalashnikova, Charly Chahwan, W Hayes McDonald, James Wohlschlegel, John Yates III, Paul Russell, and Curt Wittenberg. Constraining g1-specific transcription to late g1 phase: the mbf-associated corepressor nrm1 acts via negative feedback. Molecular cell, 23(4):483-496, 2006.

Frank Emmert-Streib, Matthias Dehmer, and Benjamin Haibe-Kains. Gene regulatory networks and their applications: understanding biological and medical problems in terms of networks. Frontiers in cell and developmental biology, 2:38, 2014.

Thomas S Ferguson. A Bayesian analysis of some nonparametric problems. The annals of statistics, pages 209-230, 1973.

Wolfgang Fischle, Yanming Wang, and C David Allis. Histone and chromatin cross-talk. Current opinion in cell biology, 15(2):172-183, 2003.

Edward W Forgy. Cluster analysis of multivariate data: efficiency versus interpretability of classifications. biometrics, 21:768-769, 1965.

Chris Fraley and Adrian E Raftery. Model-based clustering, discriminant analysis, and density estimation. Journal of the American statistical Association, 97(458):611-631, 2002. 
Jerome H Friedman. Stochastic gradient boosting. Computational statistics \& data analysis, 38(4):367-378, 2002.

Arno Fritsch. mcclust: process an MCMC sample of clusterings, 2012. URL https://CRAN.R-project. org/package=mcclust. R package version 1.0.

Arno Fritsch, Katja Ickstadt, et al. Improved criteria for clustering based on the posterior similarity matrix. Bayesian analysis, 4(2):367-391, 2009.

Evelina Gabasova, John Reid, and Lorenz Wernisch. Clusternomics: Integrative context-dependent clustering for heterogeneous datasets. PLoS computational biology, 13(10):e1005781, 2017.

Andrew Gelman, Donald B Rubin, et al. Inference from iterative simulation using multiple sequences. Statistical science, 7(4):457-472, 1992.

John Geweke et al. Evaluating the accuracy of sampling-based approaches to the calculation of posterior moments, volume 196. Federal Reserve Bank of Minneapolis, Research Department Minneapolis, MN, 1991.

Reza Ghaemi, Md Nasir Sulaiman, Hamidah Ibrahim, Norwati Mustapha, et al. A survey: clustering ensembles techniques. World Academy of Science, Engineering and Technology, 50:636-645, 2009.

Marina V Granovskaia, Lars J Jensen, Matthew E Ritchie, Joern Toedling, Ye Ning, Peer Bork, Wolfgang Huber, and Lars M Steinmetz. High-resolution transcription atlas of the mitotic cell cycle in budding yeast. Genome biology, 11(3):1-11, 2010.

Zuguang Gu, Matthias Schlesner, and Daniel Hübschmann. cola: an R/Bioconductor package for consensus partitioning through a general framework. Nucleic Acids Research, 12 2020. ISSN 0305-1048. doi: 10.1093/nar/gkaa1146. URL/https://doi.org/10.1093/nar/gkaa1146. gkaa1146.

Christopher T Harbison, D Benjamin Gordon, Tong Ihn Lee, Nicola J Rinaldi, Kenzie D Macisaac, Timothy W Danford, Nancy M Hannett, Jean-Bosco Tagne, David B Reynolds, Jane Yoo, et al. Transcriptional regulatory code of a eukaryotic genome. Nature, 431(7004):99-104, 2004.

Boris P Hejblum, Jason Skinner, and Rodolphe Thiébaut. Time-course gene set analysis for longitudinal gene expression data. PLoS computational biology, 11(6):e1004310, 2015.

Boris P Hejblum, Chariff Alkhassim, Raphael Gottardo, François Caron, Rodolphe Thiébaut, et al. Sequential Dirichlet process mixtures of multivariate skew $t$-distributions for model-based clustering of flow cytometry data. The Annals of Applied Statistics, 13(1):638-660, 2019.

Lawrence Hubert and Phipps Arabie. Comparing partitions. Journal of classification, 2(1):193-218, 1985. 
BP Ingalls, BP Duncker, DR Kim, and BJ McConkey. Systems level modeling of the cell cycle using budding yeast. Cancer informatics, 3:117693510700300020, 2007.

Vishwanath R Iyer, Christine E Horak, Charles S Scafe, David Botstein, Michael Snyder, and Patrick O Brown. Genomic binding sites of the yeast cell-cycle transcription factors SBF and MBF. Nature, 409 (6819):533-538, 2001.

Javier Jiménez, Samuel Bru, Mariana Ribeiro, and Josep Clotet. Live fast, die soon: cell cycle progression and lifespan in yeast cells. Microbial Cell, 2(3):62, 2015.

Christopher R John, David Watson, Dominic Russ, Katriona Goldmann, Michael Ehrenstein, Costantino Pitzalis, Myles Lewis, and Michael Barnes. M3C: Monte Carlo reference-based consensus clustering. Scientific reports, 10(1):1-14, 2020.

Paul Kirk, Jim E Griffin, Richard S Savage, Zoubin Ghahramani, and David L Wild. Bayesian correlated clustering to integrate multiple datasets. Bioinformatics, 28(24):3290-3297, 2012.

Vladimir Yu Kiselev, Kristina Kirschner, Michael T Schaub, Tallulah Andrews, Andrew Yiu, Tamir Chandra, Kedar N Natarajan, Wolf Reik, Mauricio Barahona, Anthony R Green, et al. SC3: consensus clustering of single-cell RNA-seq data. Nature methods, 14(5):483-486, 2017.

Alp Kucukelbir, Dustin Tran, Rajesh Ranganath, Andrew Gelman, and David M Blei. Automatic differentiation variational inference. The Journal of Machine Learning Research, 18(1):430-474, 2017.

Jessica P Lao, Katie M Ulrich, Jeffrey R Johnson, Billy W Newton, Ajay A Vashisht, James A Wohlschlegel, Nevan J Krogan, and David P Toczyski. The yeast DNA damage checkpoint kinase Rad53 targets the exoribonuclease, Xrn1. G3: Genes, Genomes, Genetics, 8(12):3931-3944, 2018.

Martin H Law, Anil K Jain, and Mário Figueiredo. Feature selection in mixture-based clustering. In Advances in neural information processing systems, pages 641-648, 2003.

Brian D Lehmann, Joshua A Bauer, Xi Chen, Melinda E Sanders, A Bapsi Chakravarthy, Yu Shyr, Jennifer A Pietenpol, et al. Identification of human triple-negative breast cancer subtypes and preclinical models for selection of targeted therapies. The Journal of clinical investigation, 121(7):2750-2767, 2011.

Stuart Lloyd. Least squares quantization in pcm. IEEE transactions on information theory, 28(2):129-137, 1982.

Gael M Martin, David T Frazier, and Christian P Robert. Computing Bayes: Bayesian Computation from 1763 to the 21st Century. arXiv preprint arXiv:2004.06425, 2020.

Mario Medvedovic and Siva Sivaganesan. Bayesian infinite mixture model based clustering of gene expression profiles. Bioinformatics, 18(9):1194-1206, 2002. 
Gunjan D Mehta, Ravinder Kumar, Sanjeeva Srivastava, and Santanu Kumar Ghosh. Cohesin: functions beyond sister chromatid cohesion. FEBS letters, 587(15):2299-2312, 2013.

Nicolai Meinshausen and Peter Bühlmann. Stability selection. Journal of the Royal Statistical Society: Series B (Statistical Methodology), 72(4):417-473, 2010.

Jeffrey W Miller and Matthew T Harrison. Mixture models with a prior on the number of components. Journal of the American Statistical Association, 113(521):340-356, 2018.

Stefano Monti, Pablo Tamayo, Jill Mesirov, and Todd Golub. Consensus clustering: a resampling-based method for class discovery and visualization of gene expression microarray data. Machine learning, 52 (1-2):91-118, 2003.

Sandhya Prabhakaran, Elham Azizi, Ambrose Carr, and Dana Pe'er. Dirichlet process mixture model for correcting technical variation in single-cell gene expression data. In International Conference on Machine Learning, pages 1070-1079, 2016.

Sylvia Richardson and Peter J Green. On Bayesian analysis of mixtures with an unknown number of components. Journal of the Royal Statistical Society: series B, 59(4):731-792, 1997.

Christian P Robert, Víctor Elvira, Nick Tawn, and Changye Wu. Accelerating mcmc algorithms. Wiley Interdisciplinary Reviews: Computational Statistics, 10(5):e1435, 2018.

Judith Rousseau and Kerrie Mengersen. Asymptotic behaviour of the posterior distribution in overfitted mixture models. Journal of the Royal Statistical Society: Series B (Statistical Methodology), 73(5): 689-710, 2011.

Gideon Schwarz et al. Estimating the dimension of a model. The annals of statistics, 6(2):461-464, 1978.

Luca Scrucca, Michael Fop, Brendan T. Murphy, and Adrian E. Raftery. mclust 5: clustering, classification and density estimation using Gaussian finite mixture models. The R Journal, 8(1):289-317, 2016. URL https://doi.org/10.32614/RJ-2016-021.

Samuel Sanford Shapiro and Martin B Wilk. An analysis of variance test for normality (complete samples). Biometrika, 52(3/4):591-611, 1965.

Itamar Simon, John Barnett, Nancy Hannett, Christopher T Harbison, Nicola J Rinaldi, Thomas L Volkert, John J Wyrick, Julia Zeitlinger, David K Gifford, Tommi S Jaakkola, et al. Serial regulation of transcriptional regulators in the yeast cell cycle. Cell, 106(6):697-708, 2001.

Chris Stark, Bobby-Joe Breitkreutz, Teresa Reguly, Lorrie Boucher, Ashton Breitkreutz, and Mike Tyers. Biogrid: a general repository for interaction datasets. Nucleic acids research, 34(suppl_1):D535-D539, 2006. 
Magdalena E Strauss, Paul DW Kirk, John E Reid, and Lorenz Wernisch. Gpseudoclust: deconvolution of shared pseudo-profiles at single-cell resolution. Bioinformatics, 36(5):1484-1491, 2020.

Attila Tóth, Rafal Ciosk, Frank Uhlmann, Marta Galova, Alexander Schleiffer, and Kim Nasmyth. Yeast cohesin complex requires a conserved protein, Ecolp (Ctf7), to establish cohesion between sister chromatids during DNA replication. Genes \& development, 13(3):320-333, 1999.

Richard Eric Turner and Maneesh Sahani. Two problems with variational expectation maximisation for time-series models. In David Barber, A Taylan Cemgil, and Silvia Chiappa, editors, Bayesian time series models, chapter 5. Cambridge University Press, 1 edition, 2011.

John J. Tyson, Katherine C. Chen, and Béla Novák. Cell cycle, budding yeast. In Werner Dubitzky, Olaf Wolkenhauer, Kwang-Hyun Cho, and Hiroki Yokota, editors, Encyclopedia of Systems Biology, pages 337-341. Springer New York, New York, NY, 2013.

Dootika Vats and Christina Knudson. Revisiting the gelman-rubin diagnostic. arXiv preprint arXiv:1812.09384, 2018.

Roel GW Verhaak, Katherine A Hoadley, Elizabeth Purdom, Victoria Wang, Yuan Qi, Matthew D Wilkerson, C Ryan Miller, Li Ding, Todd Golub, Jill P Mesirov, et al. Integrated genomic analysis identifies clinically relevant subtypes of glioblastoma characterized by abnormalities in pdgfra, idh1, egfr, and nf1. Cancer cell, 17(1):98-110, 2010.

Ulrike Von Luxburg and Shai Ben-David. Towards a statistical theory of clustering. In Pascal workshop on statistics and optimization of clustering, pages 20-26. Citeseer, 2005.

Lianming Wang and David B Dunson. Fast Bayesian inference in Dirichlet process mixture models. Journal of Computational and Graphical Statistics, 20(1):196-216, 2011.

Wilkerson, Matthew D., Hayes, and D. Neil. ConsensusClusterPlus: a class discovery tool with confidence assessments and item tracking. Bioinformatics, 26(12):1572-1573, 2010.

Svante Wold, Kim Esbensen, and Paul Geladi. Principal component analysis. Chemometrics and intelligent laboratory systems, 2(1-3):37-52, 1987.

Yuling Yao, Aki Vehtari, and Andrew Gelman. Stacking for non-mixing Bayesian computations: the curse and blessing of multimodal posteriors. arXiv preprint arXiv:2006.12335, 2020. 


\section{${ }_{515}$ Additional Files}

\section{Additional file 1 - Supplementary materials}

Additional relevant theory, background and results. This includes some more formal definitions, details of Bayesian mixture models and MDI, the general consensus clustering algorithm, additional simulations and the generating algorithm used, steps in assessing Bayesian model convergence in both the simulated datasets and yeast analysis, a table of the transcription factors that define the clustering in the ChIP-chip dataset, a table of the gene descriptions for some of the clusters that emerge across the time course and ChIP-chip datasets and Gene Ontology term over-representation analysis of the clusterings from the yeast datasets. (PDF, 10MB) 


\title{
Consensus clustering for Bayesian mixture models: Supplementary materials
}

\author{
Stephen Coleman, Paul DW Kirk, and Chris Wallace
}

February 16, 2021

\begin{abstract}
Some definitions, descriptions of mixture models and Multiple Dataset Integration, the consensus clustering algorithm, the simulation scenarios, and decisions made in the analysis pipelines. Greater details of the results from the simulations and the multi-omics analysis are also provided.
\end{abstract}

\section{Definitions}

Definition 1 (Coclustering matrix) The coclustering matrix describes a clustering or partition in a binary matrix with the $(i, j)^{\text {th }}$ entry indicating if items $i$ and $j$ are allocated to the same cluster.

Definition 2 (Consensus matrix) Given $W$ clusterings for a dataset of $N$ items, $c_{s}=\left(c_{s 1}, \ldots, c_{s N}\right)$, the consensus matrix is a $N \times N$ matrix where the $(i, j)^{\text {th }}$ entry records the proportions of clusterings for which items $i$ and $j$ are allocated the same label. More formally, it is the matrix $\mathbb{C}$ such that

$$
\mathbb{C}(i, j)=\frac{1}{W} \sum_{s=1}^{W} \mathbf{I}\left(c_{s i}=c_{s j}\right)
$$

where $\mathbb{I}(\cdot)$ is the indicator function taking a value of 1 if the argument is true and 0 otherwise.

Definition 3 (Posterior similarity matrix) A consensus matrix for which all the clusterings are generated from a converged Markov chain for some Bayesian clustering model. Sometimes abbreviated to PSM.

Definition 4 (Partition or Clustering) For a dataset of items $X=\left(x_{1}, \ldots, x_{N}\right)$, a partition or clustering is a set of disjoint sets covering $X$, normally indicated by a $N$-vector of integers indicating which set each item is associated with. Note that these labels only have meaning relative to each other, they are symbolic. Each set within the clustering is referred to as a cluster.

\section{The models}

\subsection{Individual dataset}

In the simulations (see section 4) where individual datasets are modelled, a Bayesian mixture model is used. We write the basic mixture model for independent items $X=\left(x_{1}, \ldots, x_{N}\right)$ as

$$
x_{n} \sim \sum_{k=1}^{K} \pi_{k} f\left(x_{n} \mid \theta_{k}\right) \quad \text { independently for } n=1, \ldots, N
$$

where $f(\cdot \mid \theta)$ is some family of densities parametrised by $\theta$. A common choice is the Gaussian density function, with $\theta=\left(\mu, \sigma^{2}\right)$ (as in our simulation study). $K$, the number of subgroups in the population, $\left\{\theta_{k}\right\}_{k=1}^{K}$, the component parameters, and $\pi=\left(\pi_{1}, \ldots, \pi_{K}\right)$, the component weights are the objects to be inferred. In the context of clustering, such a model arises due to the belief that the population from which the random 
sample under analysis has been drawn consists of $K$ unknown groups proportional to $\pi$. In this setting it is natural to include a latent allocation variable, $c=\left(c_{1}, \ldots, c_{N}\right)$, to indicate which group each item is drawn from, with each non-empty component of the mixture corresponds to a cluster. The model is

$$
\begin{aligned}
p\left(c_{n}=k\right)=\pi_{k} & \text { for } k=1, \ldots, K, \\
x_{n} \mid c_{n} \sim f\left(x_{n} \mid \theta_{k}\right) & \text { independently for } n=1, \ldots, N .
\end{aligned}
$$

The joint model can then be written

$$
p(X, c, K, \pi, \theta)=p(X \mid c, \pi, K, \theta) p(\theta \mid c, \pi, K) p(c \mid \pi, K) p(\pi \mid K) p(K)
$$

We assume conditional independence between certain parameters such that the model reduces to

$$
p(X, c, \theta, \pi, K)=p(\pi \mid K) p(\theta \mid K) p(K) \prod_{n=1}^{N} p\left(x_{n} \mid c_{n}, \theta_{c_{n}}\right) p\left(c_{n} \mid \pi, K\right) .
$$

Additional flexibility is provided by the inclusion of hyperparameters on the priors for $\pi$ and $\theta$, denoted $\alpha$ and $\eta$ respectively. In our context where $\theta=\left(\mu, \sigma^{2}\right)$, we use

$$
\begin{aligned}
\sigma^{2} & \sim \Gamma^{-1}(a, b), \\
\mu & \sim \mathcal{N}\left(\xi, \frac{1}{\lambda} \sigma^{2}\right), \\
\pi & \sim \operatorname{Dirichlet}(\alpha) .
\end{aligned}
$$

The directed acyclic graph (DAG) for this model is shown in figure 1. The value of the hyperparameters we use are

$$
\begin{aligned}
\alpha & =1, \\
\xi & =0.0, \\
\lambda & =1.0, \\
a & =2.0, \\
b & =2.0 .
\end{aligned}
$$

\subsection{Integrative clustering}

We are interested in the use of consensus clustering for integrative methods. We used Multiple Dataset Integration (MDI, Kirk et al., 2012) as an example of a Bayesian integrative clustering method. MDI models dataset specific clusterings, in contrast to, for example, Clusternomics (Gabasova et al., 2017) in which a global clustering is inferred.

The defining aspect of MDI is the prior on the allocation of the $n^{t h}$ item across the $L$ datasets

$$
p\left(c_{n 1}, \ldots, c_{n L}\right) \propto \prod_{l=1}^{L} \pi_{c_{n l} l} \prod_{l=1}^{L-1} \prod_{m=l+1}^{L}\left(1+\phi_{l m} \mathbb{I}\left(c_{n l}=c_{n m}\right)\right) \text { for } n=1, \ldots, N .
$$

$\phi_{l m}$ is the parameter defined by the similarity of the clusterings for the $l^{t h}$ and $m^{t h}$ datasets and is also sampled in each iteration. As $\phi_{l m}$ increases more mass is placed on the common partition for these datasets. Conversely, in the limit $\phi_{l m} \rightarrow 0$ we have independent mixture models. In other words, MDI allows datasets with similar clustering of the items to inform the clustering in each other more strongly than the clustering for an unrelated dataset. The DAG for this model for three datasets is shown in figure 2 .

\section{Consensus clustering}

Consensus clustering as described by Monti et al. (2003) applies $W$ independent runs of the underlying clustering algorithm to perturbed versions of the dataset and combines the $W$ final partitions in a consensus matrix which can be used to infer a final clustering. An outline of this is described in algorithm 1. 


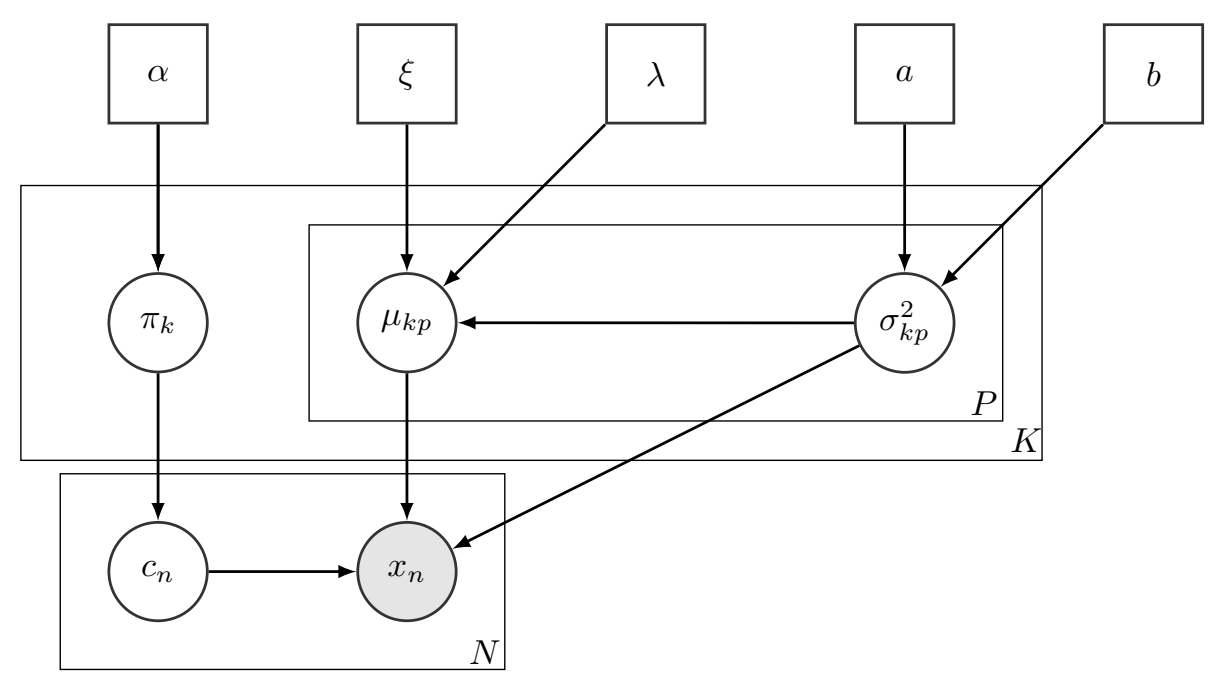

Figure 1: Directed acyclic graph for a mixture of Gaussians with independent features, as used in the simulation study.

Data: $X=\left(x_{1}, \ldots, x_{N}\right)$

Input: A resampling scheme Resample

A clustering algorithm Cluster

Number of resampling iterations $W$

Set of cluster numbers to try $\mathcal{K}=\left\{K_{1}, \ldots, K_{\max }\right\}$

Output: A predicted clustering, $\hat{Y}$

The predicted number of clusters present $\hat{K}$

begin

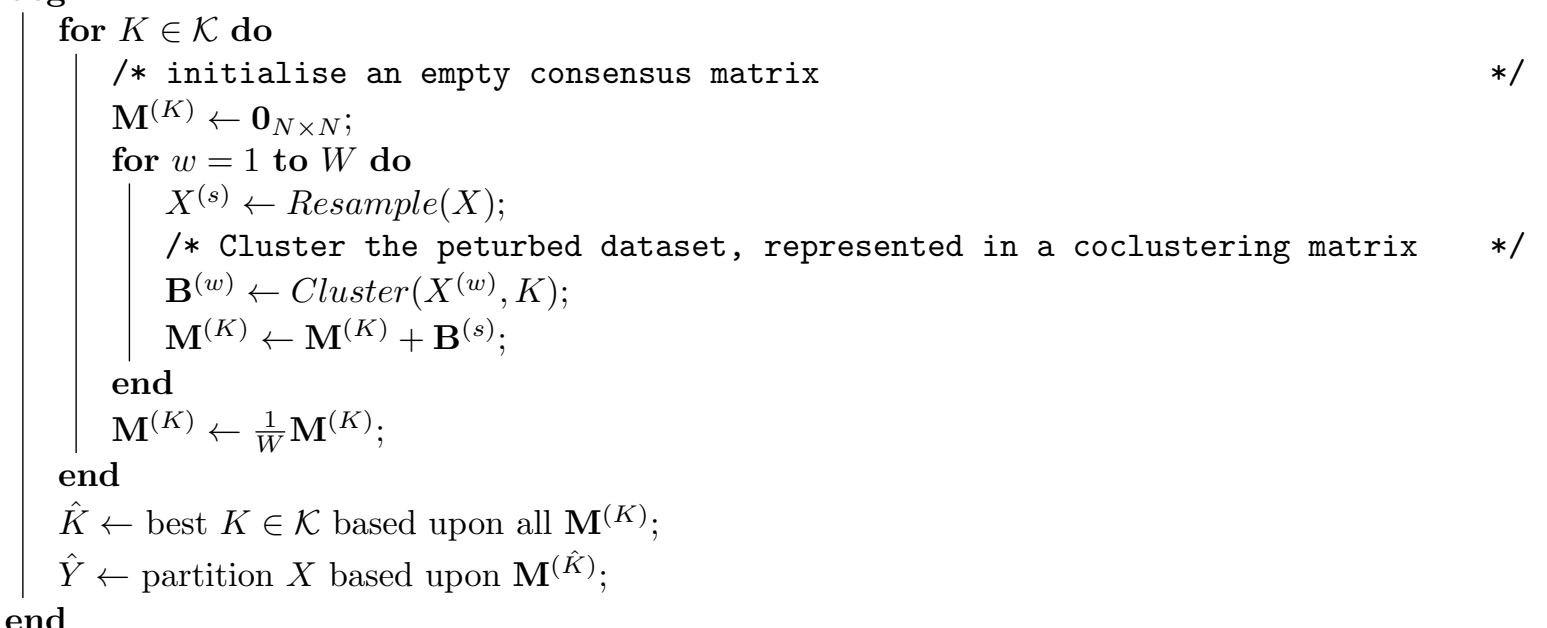

end

Algorithm 1: Consensus clustering algorithm 


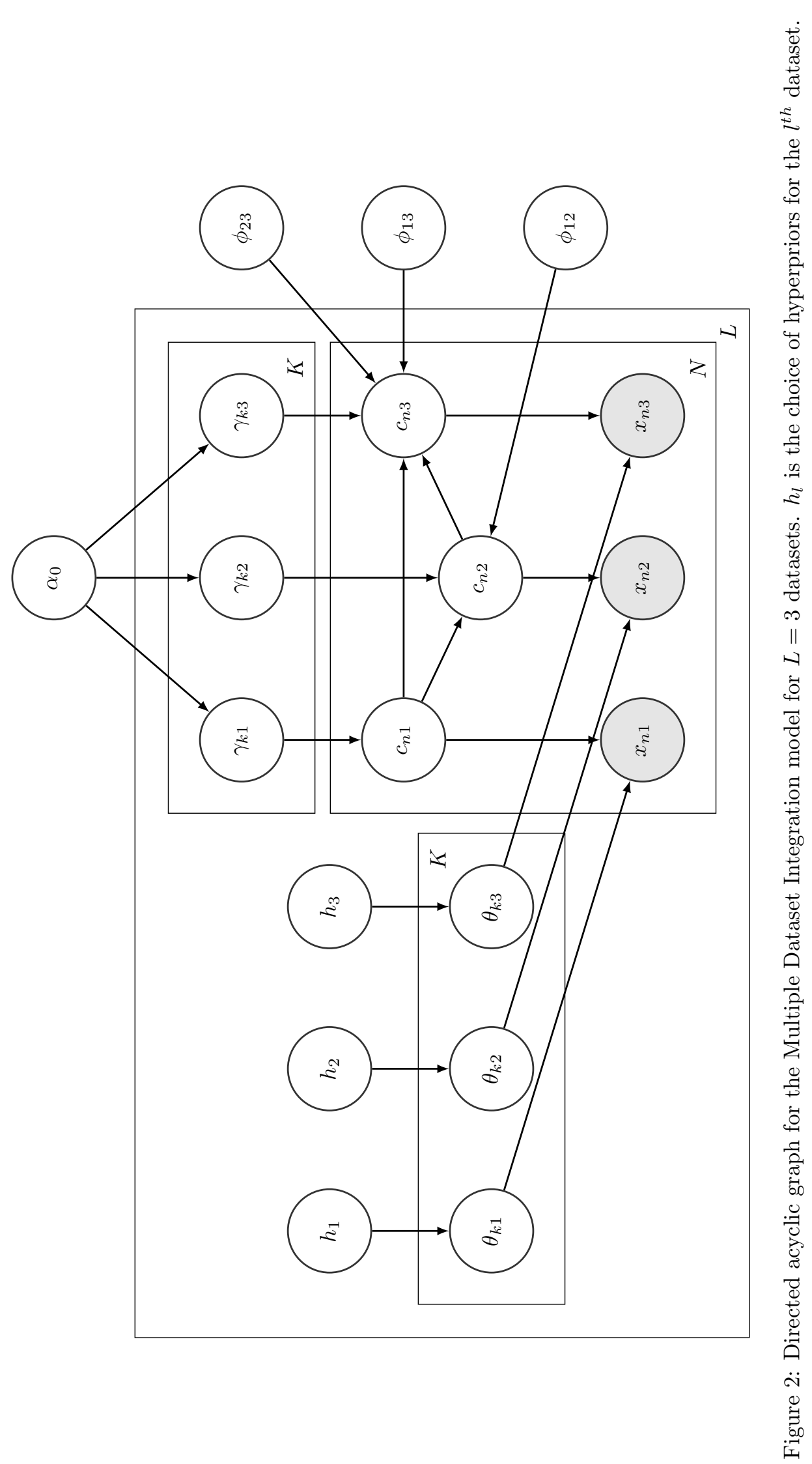


The consensus matrix is a symmetric matrix with the $(i, j)^{t h}$ entry being the proportions of model runs for which the $i^{t h}$ and $j^{t h}$ items are clustered together.

To partition $X$ based upon the consensus matrix, we use the $\mathrm{R}$ function maxpear (Fritsch, 2012). maxpear uses a sample average clustering, inferring this by maximising the quantity

$$
\frac{\sum_{i<j} \mathbb{I}\left(c_{i}^{*}=c_{j}^{*}\right) p_{i j}-\sum_{i<j} \mathbb{I}\left(c_{i}^{*}=c_{j}^{*}\right) \sum_{i<j} p_{i j} /\left(\begin{array}{c}
N \\
2
\end{array}\right)}{\frac{1}{2}\left[\sum_{i<j} \mathbb{I}\left(c_{i}^{*}=c_{j}^{*}\right)+\sum_{i<j} p_{i j}\right]-\sum_{i<j} \mathbb{I}\left(c_{i}^{*}=c_{j}^{*}\right) \sum_{i<j} p_{i j} /\left(\begin{array}{c}
N \\
2
\end{array}\right)}
$$

where $p_{i j}$ is the $(i, j)^{t h}$ entry of the consensus matrix (Fritsch et al., 2009).

\section{Simulated data}

\subsection{Scenario description}

We defined 12 scenarios to simulate data within to test consensus clustering and to compare it to some alternative tools. Table 1 describes the parameters defining these scenarios and algorithm 2 describes how individual simulations were generated.

\begin{tabular}{l|ccccccc}
\hline \hline Scenario & $N$ & $P_{s}$ & $P_{n}$ & $K$ & $\Delta \mu$ & $\sigma^{2}$ & $\pi$ \\
\hline 2D & 100 & 2 & 0 & 5 & 3.0 & 1 & $\left(\frac{1}{5}, \frac{1}{5}, \frac{1}{5}, \frac{1}{5}, \frac{1}{5}\right)$ \\
No structure & 100 & 0 & 2 & 1 & 0.0 & 1 & 1 \\
Base Case & 200 & 20 & 0 & 5 & 1.0 & 1 & $\left(\frac{1}{5}, \frac{1}{5}, \frac{1}{5}, \frac{1}{5}, \frac{1}{5}\right)$ \\
Large standard deviation & 200 & 20 & 0 & 5 & 1.0 & 9 & $\left(\frac{1}{5}, \frac{1}{5}, \frac{1}{5}, \frac{1}{5}, \frac{1}{5}\right)$ \\
Large standard deviation & 200 & 20 & 0 & 5 & 1.0 & 25 & $\left(\frac{1}{5}, \frac{1}{5}, \frac{1}{5}, \frac{1}{5}, \frac{1}{5}\right)$ \\
Irrelevant features & 200 & 20 & 10 & 5 & 1.0 & 1 & $\left(\frac{1}{5}, \frac{1}{5}, \frac{1}{5}, \frac{1}{5}, \frac{1}{5}\right)$ \\
Irrelevant features & 200 & 20 & 20 & 5 & 1.0 & 1 & $\left(\frac{1}{5}, \frac{1}{5}, \frac{1}{5}, \frac{1}{5}, \frac{1}{5}\right)$ \\
Irrelevant features & 200 & 20 & 100 & 5 & 1.0 & 1 & $\left(\frac{1}{5}, \frac{1}{5}, \frac{1}{5}, \frac{1}{5}, \frac{1}{5}\right)$ \\
Varying proportions & 200 & 20 & 0 & 5 & 1.0 & 1 & $\left(\frac{1}{2}, \frac{1}{4}, \frac{1}{8}, \frac{1}{16}, \frac{1}{16}\right)$ \\
Varying proportions & 200 & 20 & 0 & 5 & 0.4 & 1 & $\left(\frac{1}{2}, \frac{1}{4}, \frac{1}{8}, \frac{1}{16}, \frac{1}{16}\right)$ \\
Small $N$, large $P$ & 50 & 500 & 0 & 5 & 1.0 & 1 & $\left(\frac{1}{5}, \frac{1}{5}, \frac{1}{5}, \frac{1}{5}, \frac{1}{5}\right)$ \\
Small $N$, large $P$ & 50 & 500 & 0 & 5 & 0.2 & 1 & $\left(\frac{1}{5}, \frac{1}{5}, \frac{1}{5}, \frac{1}{5}, \frac{1}{5}\right)$ \\
\hline
\end{tabular}

Table 1: Parameters defining the simulation scenarios as used in generating data and labels.

We intend the scenarios to test different aspects of real data or to benchmark performance for comparison in the more challenging situations.

- 2D: a low dimensional scenario within which we expected Mclust to perform well and the long chains to converge and explore the full support of the posterior distribution.

- No structure: we included this scenario to reassure fears that consensus clustering has a predilection to finding clusters where none exist (Șenbabaoğlu et al., 2014a,b).

- Base case: highly informative datasets within which we expected methods to find the true generating labels quite easily. We included this scenario to benchmark the others that are variations of this setting.

- Large standard deviation: these two scenarios investigated the degree of distinction required between clusters for the methods to uncover their structure.

- Irrelevant features: we included these scenarios to investigate how robust the methods are to irrelevant features.

- Varying proportions: these scenarios investigated how well each method uncovers clusters when the clusters have significantly different membership counts.

- Small $N$, large $P$ : an investigation of behaviour when the number of features is far greater than the number of items. 
Algorithm: Simulation generation

Input: Distance between means $\Delta_{\mu}$ A common standard deviation $\sigma^{2}$

A number of clusters $K$

The number of items to generate in total $N$

The number of features to generate in total $P$

An indicator vector of feature relevance $\phi=\left(\phi_{1}, \ldots, \phi_{P}\right)$

The expected proportion of items in each cluster $\pi=\left(\pi_{1}, \ldots, \pi_{K}\right)$

A method for sampling $x$ times from the array $y$, with weights $\pi$ : Sample $(y, x, \pi)$

A method for permuting a vector $x$ : Permute $(x)$

A method for generating a value from a univariate Gaussian distribution with mean $\mu$ and standard deviation $\sigma^{2}$ : Gaussian $\left(\mu, \sigma^{2}\right)$

Output: A dataset, $X$

The generating cluster labels $c=\left(c_{1}, \ldots, c_{N}\right)$

begin

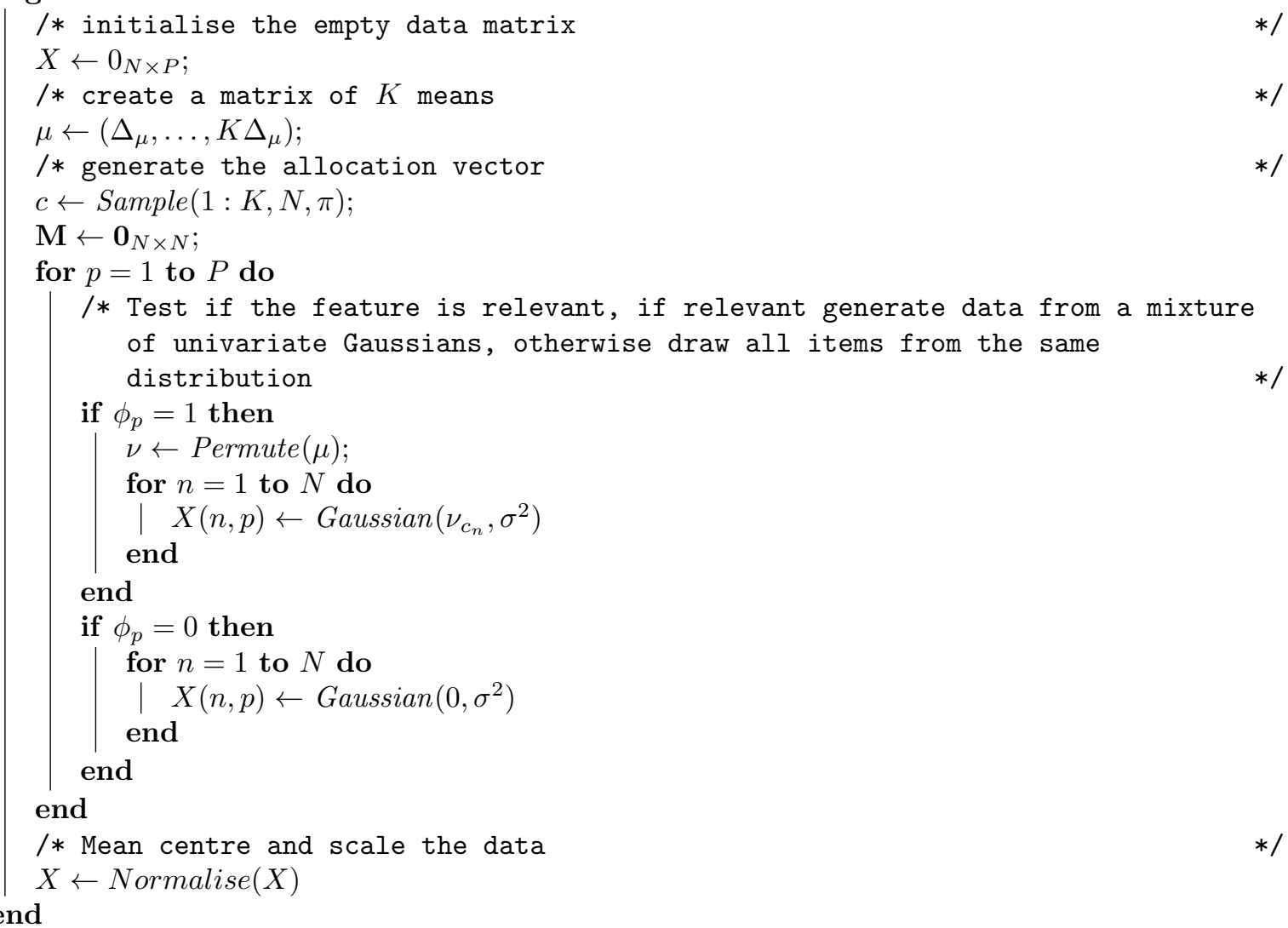

Algorithm 2: Data generation for a mixture of Gaussian with independent features. This algorithm is implemented in the generateSimulationDataset function from the mdiHelpR package available at www.github.com/stcolema/mdiHelpR. 


\subsection{Mclust}

We called Mclust using the default settings and a range of inputs for the choice of $K$. We used $K=$ $\left(2, \ldots, \min \left(\frac{N}{2}, 50\right)\right)$ to mirror the choice of $K_{\max }=50$ used for the overfitted mixture models (the default in the software we used), with the bound of $\frac{N}{2}$ to avoid fitting 50 clusters in the $S m a l l ~ N$, large $P$ scenario where $N=50=K_{\max }$. In the $N o$ structure scenarios we extended to range to $K=(1 \ldots, 50)$ to include the correct structure as an option. The model choice was performed using the Bayesian Information Criterion (Schwarz et al., 1978, as implemented in Mclust). Mclust tries different covariance matrices and thus the model choice is not just between different values of $K$.

\subsection{Bayesian analysis}

We use the implementation of Bayesian mixture models in $\mathrm{C}++$ provided by Mason et al. (2016). Rather than directly using a Dirichlet process (Ferguson, 1973) to infer the number of clusters or a mixture that grows and shrinks (Richardson and Green, 1997), this implementation follows the logic of Rousseau and Mengersen (2011) and Van Havre et al. (2015) using an overfitted mixture model to approximate a Dirichlet process. In overfitted mixture models, the number of components, $K_{\max }$, included in the model is set to number far larger than the true number of clusters, $K$, present.

For each simulation we ran 10 chains for 1 million iterations, keeping every thousandth sample. We discarded the first 10,000 iterations to account for burn-in bias, leaving 990 samples per chain. To check if the chains were converged we used

- the Geweke convergence diagnostic (Geweke et al., 1991) to investigate within-chain stationarity, and

- the potential scale reduction factor ( $\hat{R}$, Gelman et al., 1992) and the Vats-Knudson extension (stable $\hat{R}$, Vats and Knudson, 2018) to check across-chain convergence.

The Geweke convergence diagnostic is a standard Z-score; it compares the sample mean of two sets of samples (in this case buckets of samples from the first half of the samples to the sample mean of the entire second half of samples). It is calculated under the assumption that the two parts of the chain are asymptotically independent and if this assumption holds (i.e. the chain is sampling the same distribution in both samples) than the scores are expected to be standard normally distributed. If a chain's Geweke convergence diagnostic passed a Shapiro-Wilks test for normality (Shapiro and Wilk, 1965) (based upon a threshold of 0.05), we considered it to have achieved stationarity and included it in the model performance analysis.

$\hat{R}$ is expected to approach 1.0 if the set of chains are converged. Low $\hat{R}$ is not sufficient in itself to claim chain convergence, but values above 1.1 are clear evidence for a lack of convergence (Gelman et al., 2013). Vats and Knudson (2018) show that this threshold is significantly too high (1.01 being a better choice) and propose extensions to $\hat{R}$ that enable a more formal rule for a threshold. We use their method as implemented in the R package stableGR (Knudson and Vats, 2020) as the final check of convergence. An example of the $\hat{R}$ series across the 100 simulations for a scenario where chains are well-behaved is shown in figure 3 .

We focused upon stationarity of the continuous variables as assesing convergence of the allocation labels is difficult due to label-switching. In our simulations the only recorded continuous variable is the concentration parameter of the Dirichlet distribution for the component weights.

We pooled the samples from the stationary chains and used these to form a PSM. This and the point estimate clustering found by applying the $\mathrm{R}$ function maxpear. In Bayesian inference, maxpear attempts to find the clustering that maximises the Adjusted Rand Index to the true clustering by using an approximation of the expected clustering under the posterior, $\mathbb{E}(c \mid X)$, believing that this converges to the true clustering. A sample average clustering is used to approximate the expected clustering. This is estimated from the PSM by maximising

$$
\frac{\sum_{i<j} \mathbb{I}\left(c_{i}^{*}=c_{j}^{*}\right) p_{i j}-\sum_{i<j} \mathbb{I}\left(c_{i}^{*}=c_{j}^{*}\right) \sum_{i<j} p_{i j} /\left(\begin{array}{c}
N \\
2
\end{array}\right)}{\frac{1}{2}\left[\sum_{i<j} \mathbb{I}\left(c_{i}^{*}=c_{j}^{*}\right)+\sum_{i<j} p_{i j}\right]-\sum_{i<j} \mathbb{I}\left(c_{i}^{*}=c_{j}^{*}\right) \sum_{i<j} p_{i j} /\left(\begin{array}{c}
N \\
2
\end{array}\right)}
$$

where $p_{i j}$ is the $(i, j)^{t h}$ entry of the PSM (Fritsch et al., 2009). When the chain has converged this maximises the posterior expected ARI to the true clustering.

There are three possibilities to consider the decision to pool the samples across chains under: 


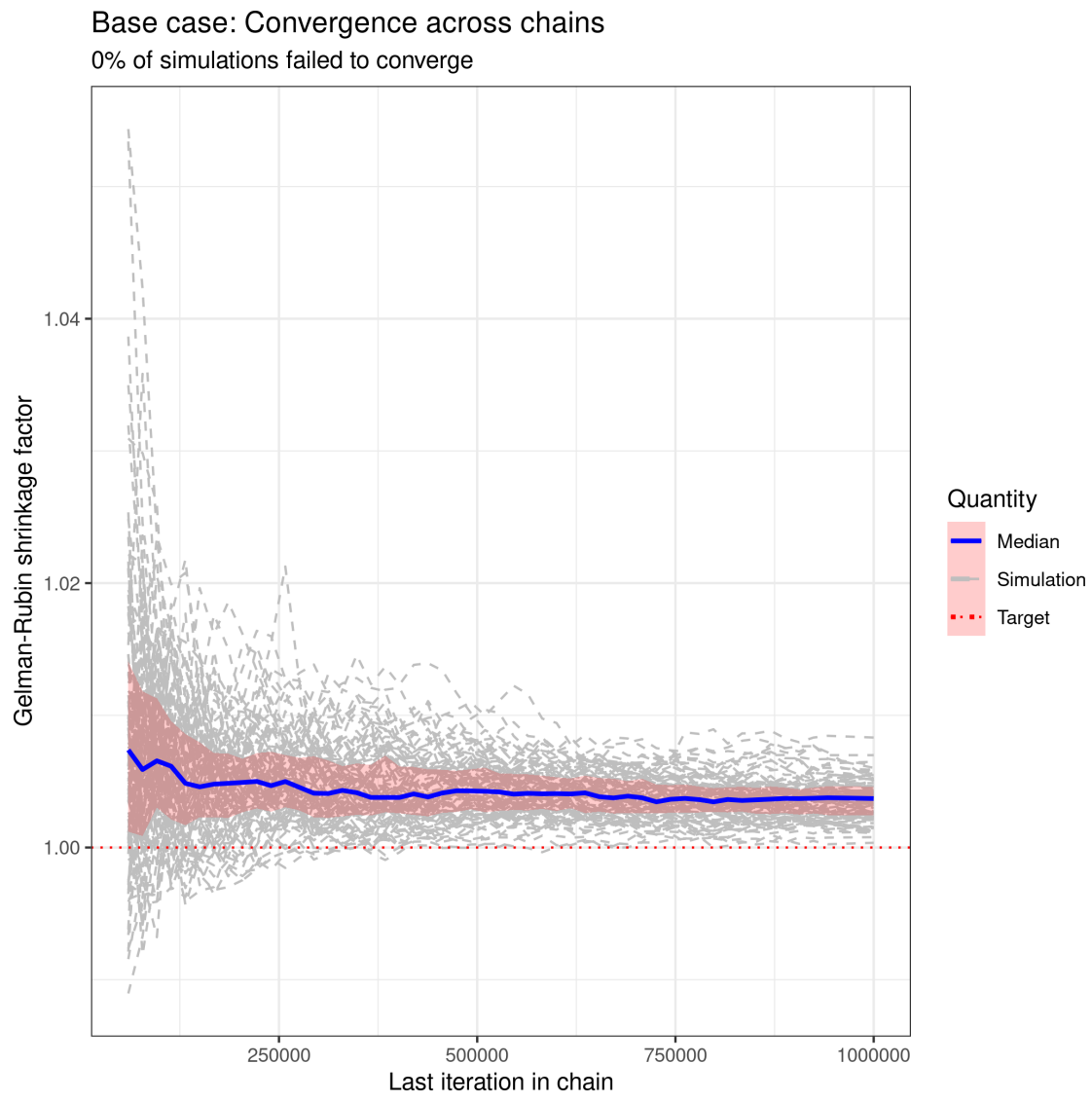

Figure 3: The $\hat{R}$ values for each simulation (in dotted grey), the median value and the interquartile range across simulations. One can see that $\hat{R}$ approaches 1.0 , being below 1.01 for every simulation by the end of the chains. The " $0 \%$ of simulations failed to converge" is a statement based upon the percentage of simulations which passed the test of stable $\hat{R}$. 


\section{Large standard deviation $\left(\sigma^{2}=9\right)$}

\section{Posterior similarity matrices (simulation 1)}
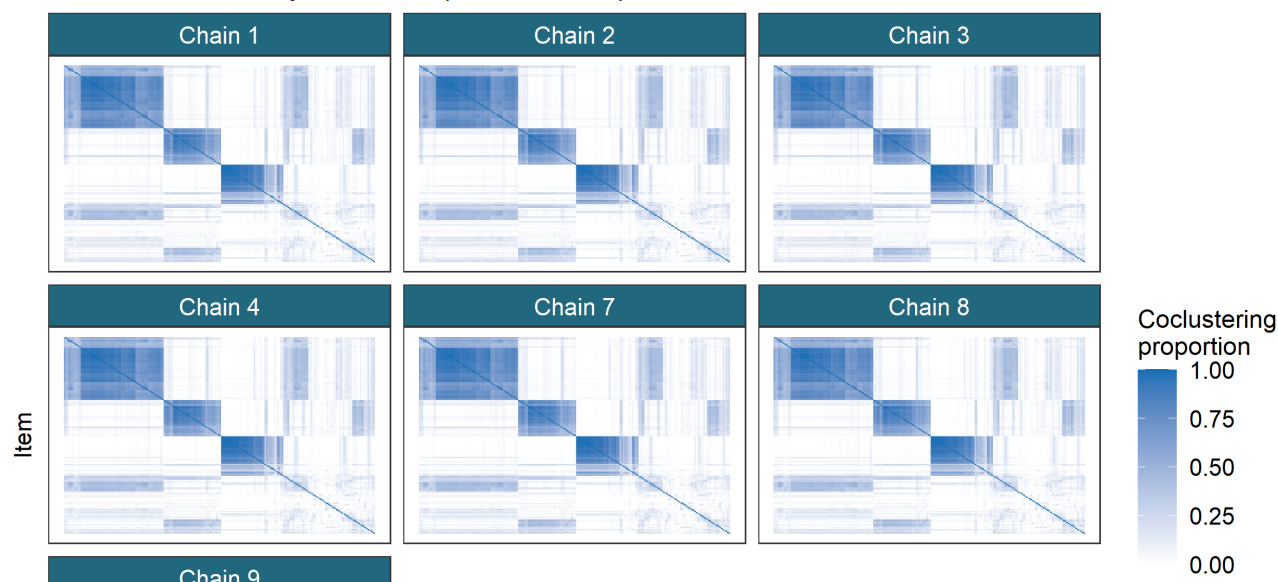

Item

Figure 4: Posterior similarity matrices for the simulation generated using a random seed set to 1 for the first large standard deviation scenario from table 1 . This is an example of all stationary chains agreeing in a simulation (and thus pooling of samples is no different to using any choice of chain for the performance analysis). Ordering of rows and columns is defined by hierarchical clustering of the first matrix in the series, in this case that from Chain 1.

- The chains are converged and agree upon the distribution sampled (see figure 4 for an example).

- The chains are not in agreement upon the partition sampled, becoming trapped in different modes. However, a mode does dominate being the mode present in a majority of chains (see figure 5 for an example of this behaviour).

- The chains are not in agreement and no one mode dominates among chains (see figure 6 for an example of this behaviour).

In the first case pooling has no effect upon the predicted clustering compared to using any one chain. In the second case it feels natural that one would use the mode that dominates. Pooling the samples effectively does this for the predictive performance of the method as the mode with the greatest number of samples across the chains dominates; however, the uncertainty for this mode is increased. In the third case the analysis is non-trivial and further thought, chains and samples would be required. In our simulations this case only arises in the most pathological form in the second Large $N$, small $P$ scenario, where each chain remains trapped in the initial partition. The clustering inferred from any chain is not meaningful being a random clustering; thus the clustering predicted by pooling the PSMs is no more or less relevant as it too is random. 


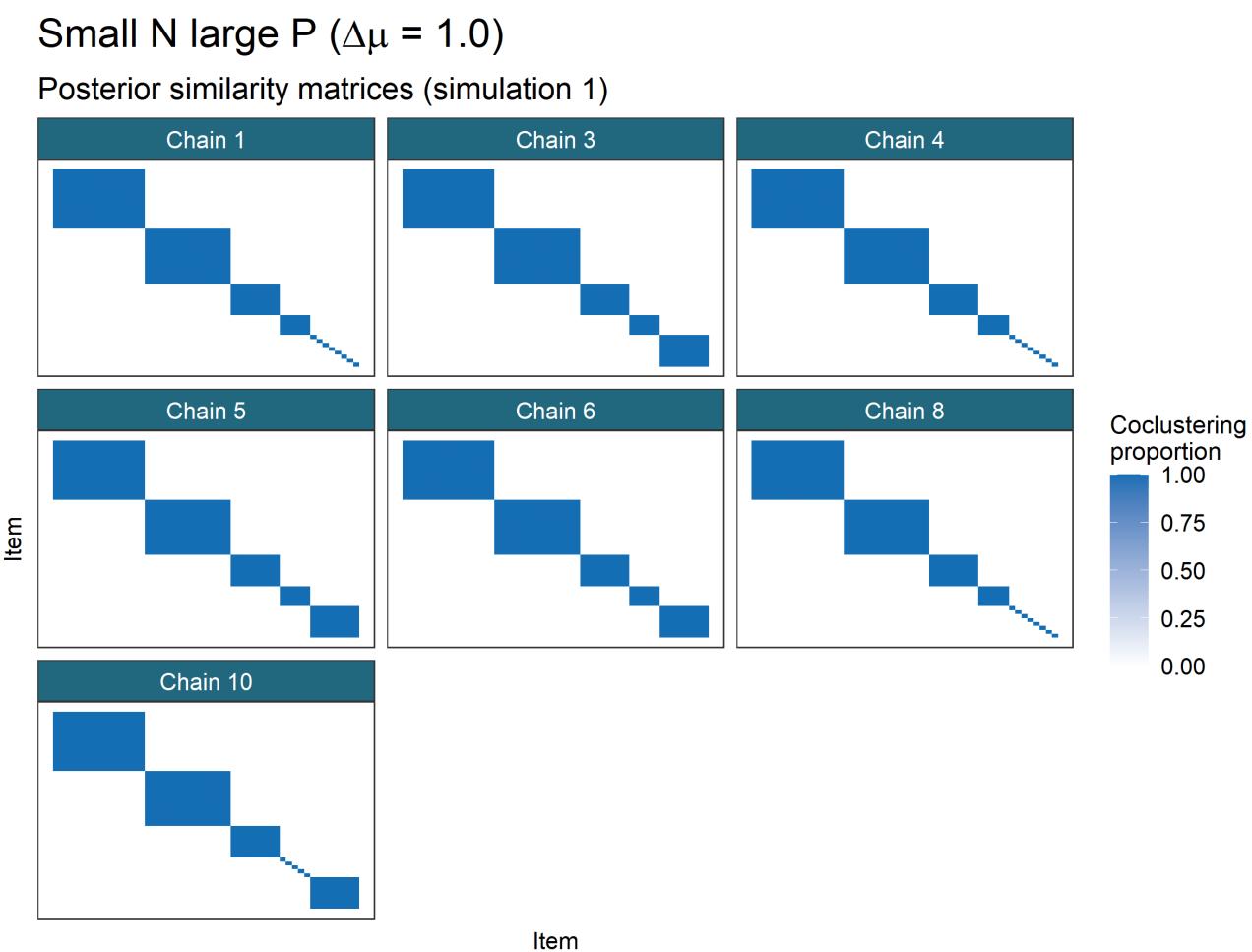

Figure 5: Posterior similarity matrices for the simulation generated using a random seed set to 1 for the first small $N$, large $P$ scenario from table 1 . This is an example of different chains becoming trapped in different modes, but one mode (which does represent the generating structure well) is dominant, being fully present in 3 of the 6 chains, with the two other modes present having significant overlap. Ordering of rows and columns is defined by hierarchical clustering of the first matrix in the series, in this case that from Chain 1. 


\section{Small N large $\mathrm{P}(\Delta \mu=0.2)$}

\section{Posterior similarity matrices (simulation 1)}
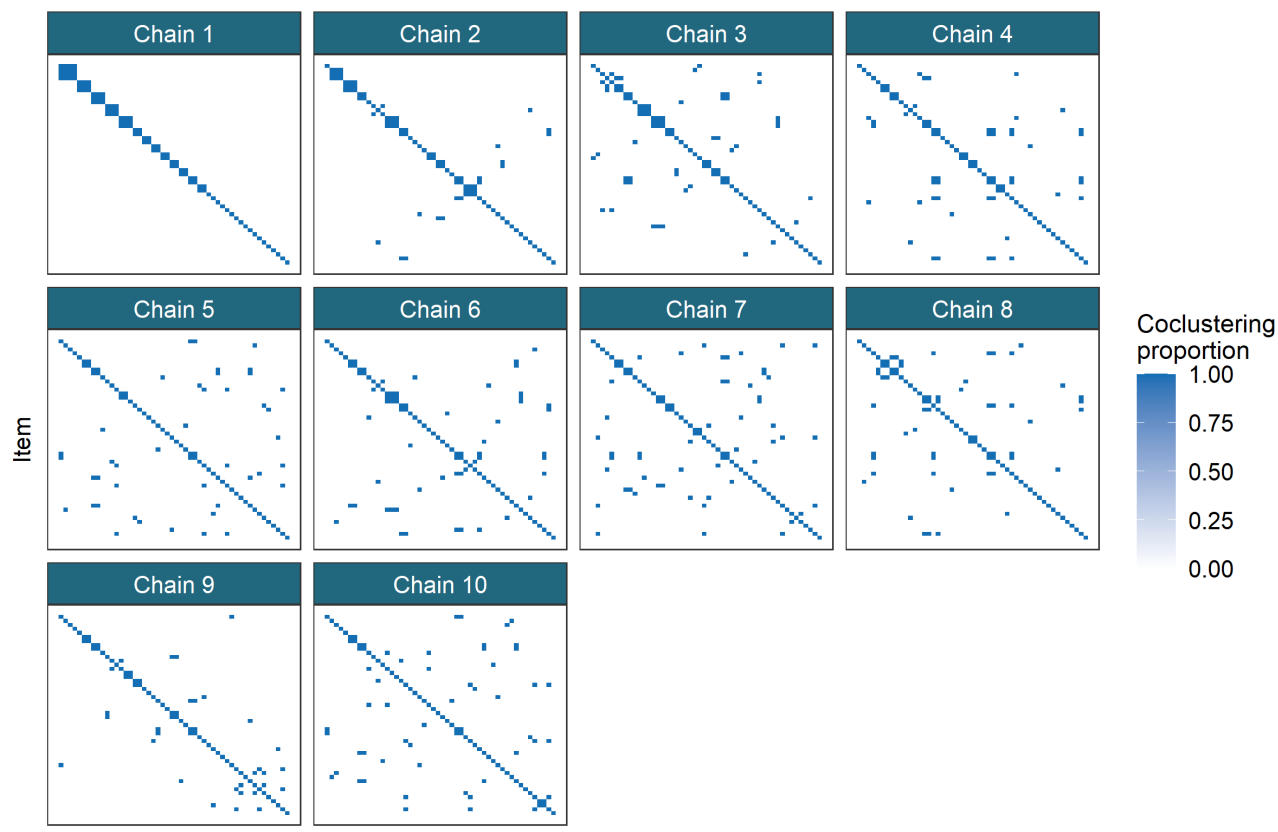

Item

Figure 6: Posterior similarity matrices for the simulation generated using a random seed set to 1 for the second small $N$, large $P$ scenario from table 1 . This is an example of different chains becoming trapped in different modes with no mode being dominant. In this scenario each chain remains trapped in initialisation. Ordering of rows and columns is defined by hierarchical clustering of the first matrix in the series, in this case that from Chain 1. 


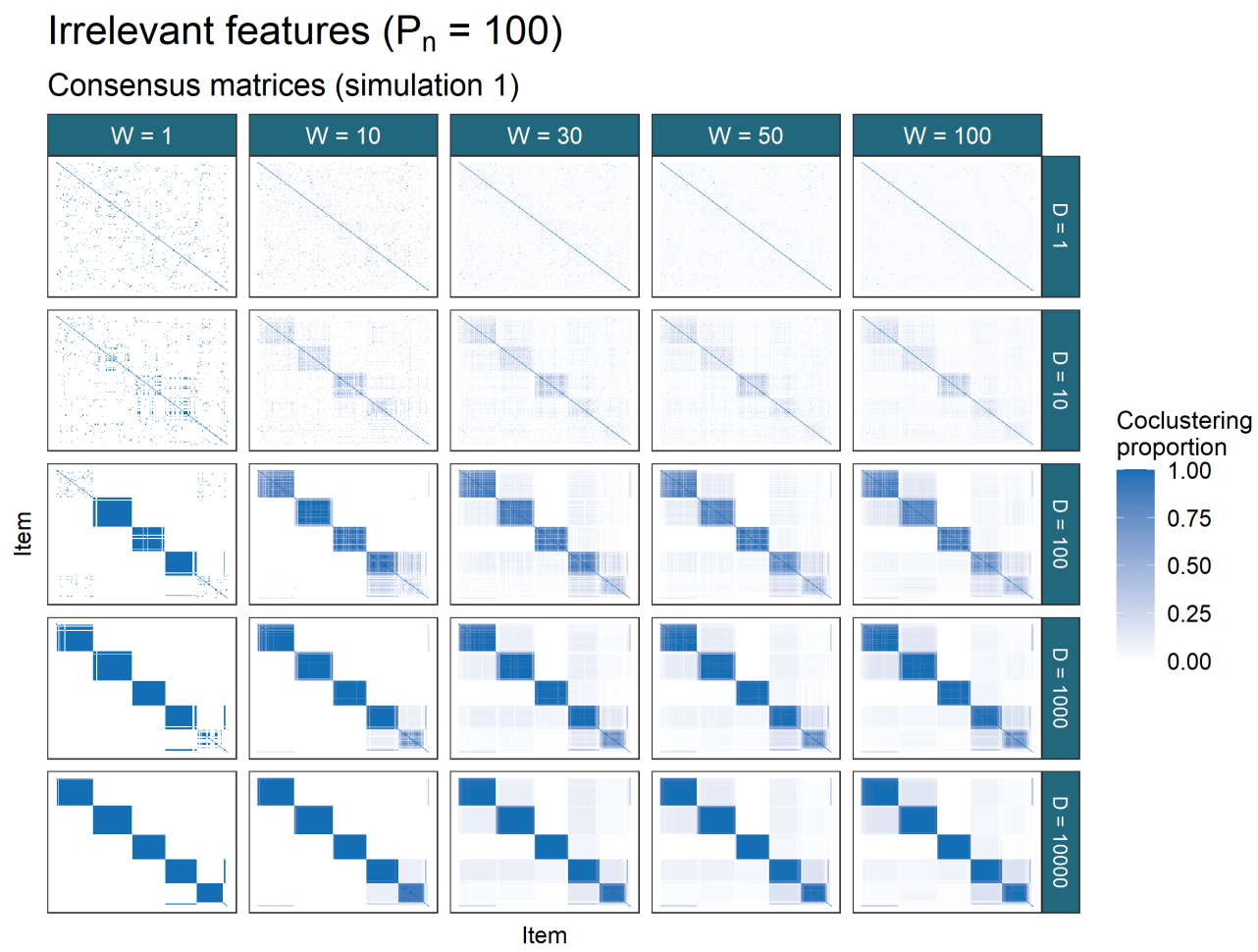

Figure 7: Consensus matrices for the simulation generated using a random seed set to 1 for the third irrelevant features scenario from table 1. $D$ is the individual chain length and $W$ is the number of chains used. In this example there are several modes present (as seen in the entries with values between 0 and 1) but one mode is clearly dominant (the 5 dark squares along the diagonal which correspond closely to the generating labels).

\subsection{Consensus clustering analysis}

We investigated a range of ensembles, using all combinations of chain depth, $D=\{1,10,100,1000,10000\}$, and the number of chains, $W=\{1,10,30,50,100\}$. This gave a total of 25 different ensembles. A consensus matrix was constructed from the samples generated by each ensemble by finding the proportion of samples within which any pair of items are coclustered. An example of the Consensus matrices for each ensemble in a given simulation is shown in figure 7 .

\subsection{Model performance}

The different models (Bayesian (pooled), Mclust and the 25 consensus clustering ensembles) were compared under their ability to uncover the generating clustering.

In figure 11 the ARI between the generating labels and the point estimate clustering from each method is shown. For two partitions $c_{1}, c_{2}$,

- $A R I\left(c_{1}, c_{2}\right)=1.0$ : a perfect match between the two partitions,

- $\operatorname{ARI}\left(c_{1}, c_{2}\right)=0.0: c_{1}$ is no more similar to $c_{2}$ than is expected for a random partition of the data.

In several scenarios Mclust performs the best under this metric (e.g. in the scenarios 2D, Small N, large $P(\Delta \mu=0.2)$ ). However when the number of irrelevant features is large Mclust performs less well (see Irrelevant features $\left(P_{n}=20\right)$ and $\left.\left(P_{n}=100\right)\right)$ than the other methods. In the scenario that $P_{n}=100$ failing to find structure is not inherently wrong as a majority of the features suggest that there are no subpopulations. 
For the ensembles there are two parameters changing between each model, the iteration used to provide the clustering in the ensemble, $D$, and the number of chains (and hence samples) used, $W$. In many of the scenarios we find that the benefit of increasing $D$ stabilises by approximately $D=10$. We believe that in a low-dimensional dataset (such as 2D), or a highly informative dataset (such as Base case or any of the higher dimensional scenarios with no irrelevant features where $\frac{\Delta \mu}{\sigma^{2}} \geq 1$ ) the chains quickly find a "sensible" partition of the data and thus increasing the depth within the chain does not increase the probability that any partition sampled will be closer to the generating partition. For example in figure 11 in the Small $N$, large $P$ case, the distribution of the ARI across the ensembles for which $D \geq 10$ and $W=1$ is nearly identical; this suggests that the chain is sampling a very similar partition again and again for 9,990 iterations (and possibly beyond based upon the PSMs shown in figure 5) and it is through adding more chains rather than using particularly long chains that we improve the ability to uncover the generating structure.

We also notice that even if the behaviour has not stabilised for $D$ that the ensemble can uncover meaningful structure. The ARI for the ensembles of short chains can be quite high (as is the case in many of the scenarios). The behaviour of the consensus matrices also shows that low $D$ is not a disqualifier from meaningful inference even if longer chains would be ideal, a result that might be useful in real applications with large datasets and complex models. Consider the consensus matrices in figure 7 , it can be seen that the behaviour has not stabilised before $D=10000$ (and possibly there is still some benefit in increasing $D$ beyond this value), but the structure being uncovered when there is a sufficient number of chains and $D$ is small does correspond to the structure uncovered in the largest and deepest ensemble. We believe that the order in which components merge and items are co-clustered varies depending on initialisation, and thus if the chain is not sufficiently deep that all of the final mergings have occured that a sufficiently large ensemble can still perform meaningful inference of the subpopulation structure despite the poor performance of any individual model. Even though each learner probably has too many clusters for small $D$ the consensus among them will have less if the individual learners have low correlation between their partitions (something we might expect if the chains are stopped very early). This is why the entries of the consensus matrix for $D=100$ and $W=100$ in figure 7 are more pale than in deeper ensembles; very few items correctly (possibly none) cocluster in every partition, it is only in observing the consensus that the global structure of interest emerges. Thus if there is some limit to the length of chains available for an analysis (e.g. computational or temporal constraints) than the inference obtained from the shorter chains can still be meaningful, with the caveat that the point clustering might have more clusters than the same analysis with longer chains would provide. Additional post-hoc merging of some clusters might be necessary in this case.

In contrast, when the dataset is sparse or contains many irrelevant features, we believe that deeper chains are required to reach this steady-state sampling where no single sample is expected to be better than any other (see the Irrelevant features $\left(P_{n}=100\right)$ facet of figure 11).

In some scenarios no method is successful in uncovering the generating labels. In the Large standard deviation $\left(\sigma^{2}=25\right)$ and Small $N$, large $P(\Delta \mu=0.2)$ this is due to the lack of signal - the clusters overlap so significantly that it is not possible for any of these methods to uncover much of the generating structure. In the No structure case it is different (although Mclust does perform well here). In this case all items are generated from a common distributions. For the Bayesian chains and the ensembles, a clustering of singletons is predicted; each item is allocated a unique label (see figures 8 and 9). While failing to perform well under the ARI, this is a sensible result. Rather than indicating (as we did with the shared label) that no item is particularly distinct from the others and thus all share a common label, this clustering of singletons states that no item is more similar to any other and thus no two items should cluster together. It is an alternative statement of the same result, i.e. that there is no evidence for subpopulation structure. We consider this evidence that an ensemble of Bayesian mixture models is not as susceptible to predicting labels than an ensemble based upon $K$-means clustering as in Șenbabaoğlu et al. (2014a,b).

Increasing $W$ is also required when the dimensionality of the dataset is large. In this case it is due to individual chains exploring only a single mode (as can be seen in figure 5 where each chain appears to sample only a single partition). In this example where each sample is a partition that appears to be a mode in the posterior distribution of the allocation vector from very early in the chain (based upon the stable performance for $D \geq 10$ ), increasing $W$ allows each chain to "vote" on which mode is the global mode, as we believe that the mode that attracts the most chains is the global mode (although in real datasets the number of chains required might be greater than in our simulations). An example of this behaviour may be seen in figure 10. 
bioRxiv preprint doi: https://doi org/10.1101/2020.12.17.423244; this version posted March 13,2021 . The copyright holder for this preprint (which was not certified by peer review) is the author/funder, who has granted bioRxiv a license to display the preprint in perpetuity. It is made available under aCC-BY 4.0 International license.

\section{No structure}

Posterior similarity matrices (simulation 1)
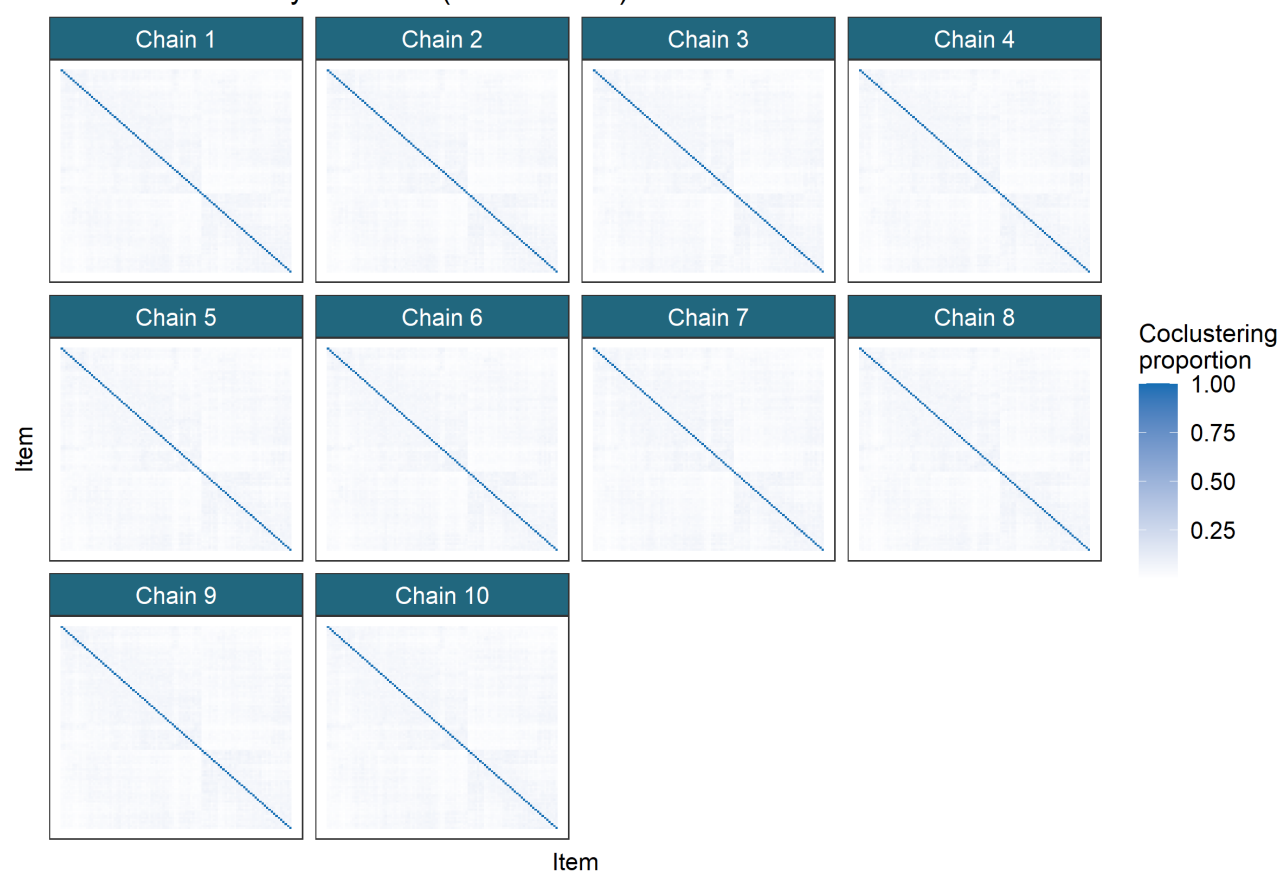

Figure 8: Posterior similarity matrices for simulation 1 of the No structure scenario. Each item is allocated to a singleton.

\section{No structure}

Consensus matrices (simulation 1)
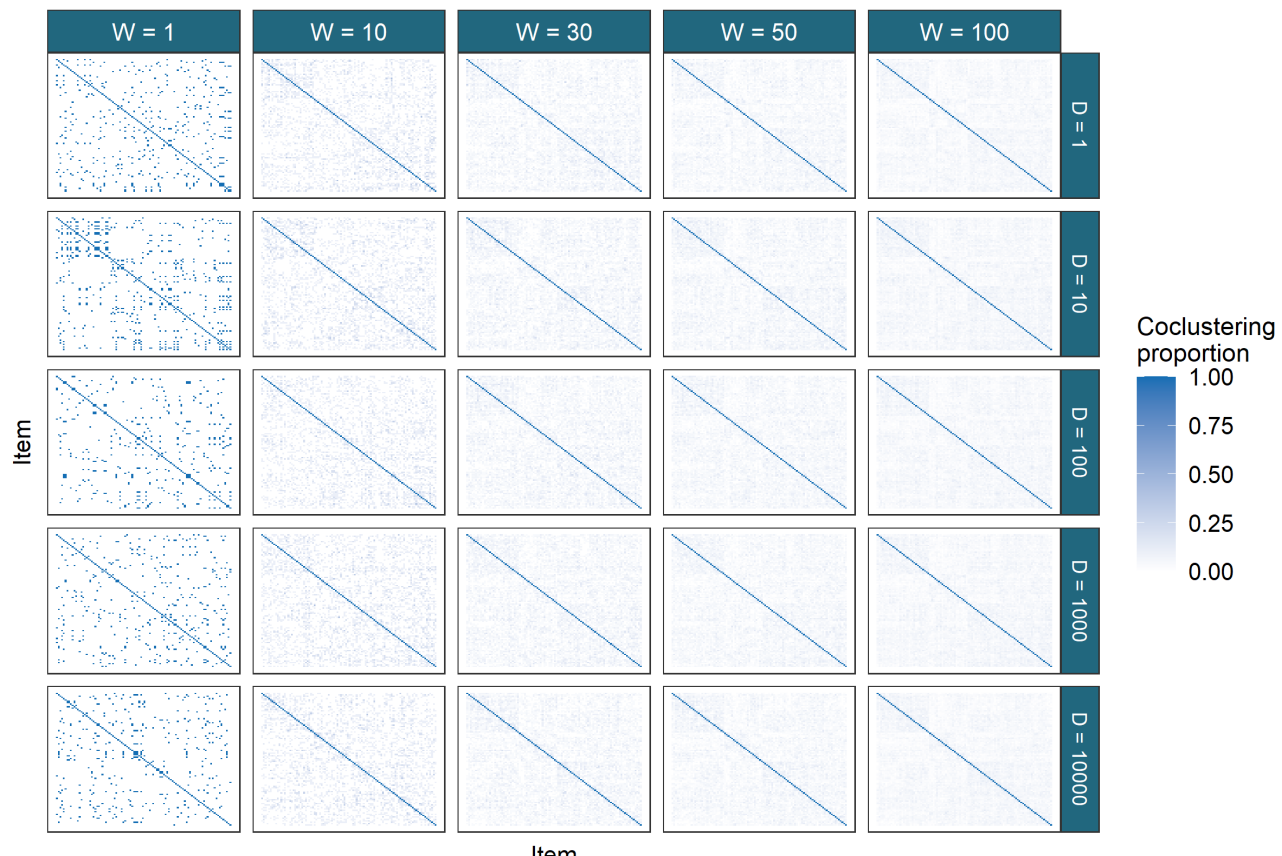

proportion
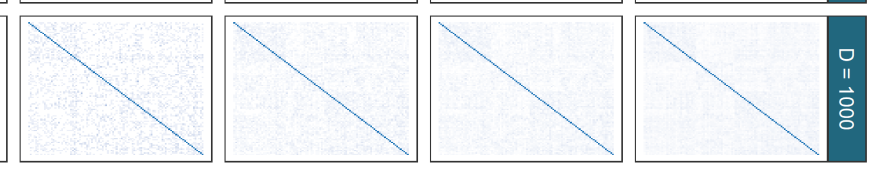

\subsection{5 \\ 0.50 \\ 0.25 \\ 0.00}

Figure 9: Consensus matrices for simulation 1 of the No structure scenario. Each item is allocated to a singleton in many of the Consensus matrices. 


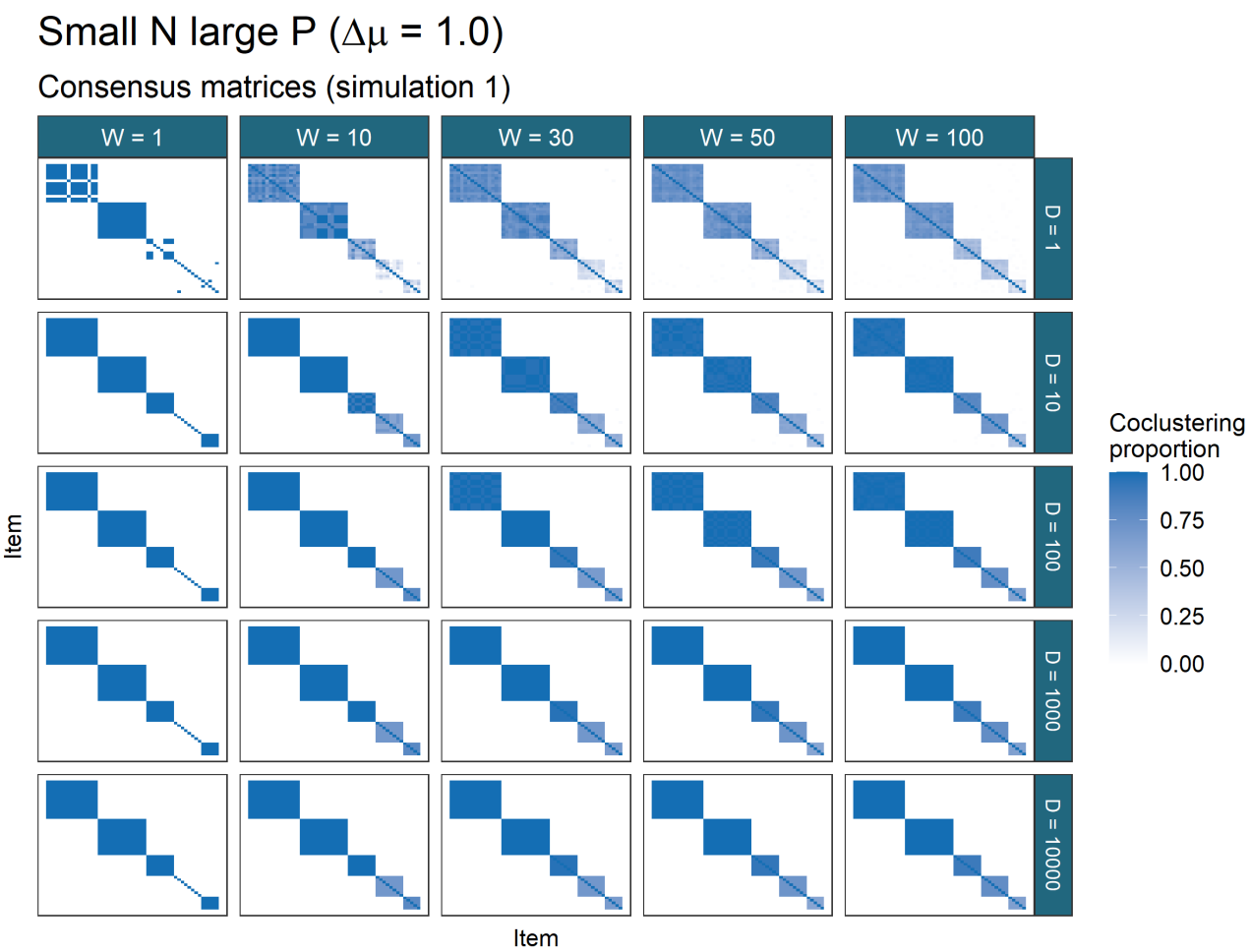

Figure 10: Consensus matrices for simulation 1 of the first Large $N$, small $P$ scenario. One can see that by iteration ten the sample being drawn is from the mode (for $W=1, D=10$ ), and that an ensemble of chains does find structure that recalls the generating labels (see figure 11, the ARI for $C C(10, s)$ is 1.0 for $s>1$, meaning that the true labels perfectly align with those predicted by the consensus matrix). 
In figure 11, limiting behaviour for increases of $W$ and $D$ can be seen for the ensemble. For most simulations there is no change in performance for greater choices of $W$ and $D$ after some stabilising values.

\section{Multi-omics analysis of the cell cycle in budding yeast}

We chose our three datasets (shown in figure 12) to perform an integrative analysis as many of the protein encoding genes in the mitotic cell cycle have well studied genomic binding sites with mapped transcription factors (TFs) that control phase-specific expression (Cho et al., 1998; Spellman et al., 1998); thus the inclusion of the ChIP-chip data means that the clusters that align across the datasets should include well studied regulatory proteins and thus be of biological interest. If a cluster of genes are similarly expressed in the time course, share associated regulatory protein in the ChIP-chip and are associated with common protein complexes in the PPI data, than this implies a gene set with strong biological significance.

In contrast, if we cluster the time course dataset alone, any clusters that we find are defined by correlation across time. This might be assumed to be driven by shared regulatory mechanisms, but other sources of structure might be encouraging this, even experimental error. However, if a cluster aligns across both the time course dataset and the ChIP-chip dataset we can be more certain that these genes are part of some regulatory network; if this cluster also emerges in the PPI dataset we might believe that the genes are co-regulated as part of the formation of some protein complex. Furthermore, this integrative aspect means that clusters that might merge in the time course dataset due to similar periodicity in a standalone analysis might remain separate due to different associated transcription factors in the ChIP-chip dataset.

Thus we performed an integrative analysis using MDI to avoid agressive assumptions about either the biology defining any clusters and modelling assumptions about the latent structure.

We expect that the complexity of this data and model means that the time required for convergence of the MCMC algorithm would be very large. We avoid this problem by using consensus clustering of MDI, instead basing our final ensemble choice on the stopping rule described in the main paper.

The datasets were modelled using a mixture of Gaussian processes in the time course dataset and Multinomial distributions in the ChIP-chip and PPI datasets. To ensure that our mixture model is initially overfitted we set $K_{\max }=275 \approx \frac{N}{2}$, and following from this the point estimate was inferred from the consensus matrix using maxpear as in the simulated data except we set $\mathrm{k} \cdot \max =275$.

\subsection{Consensus clustering analysis}

We include the consensus matrices for each dataset for a range of ensembles for further evidence that the ensemble was stable for the $10,000^{t h}$ iteration from 1,000 chains in figures 13,14 and 15 . In these figures, there is no strong change between the consensus matrices for $D=5001$ and $D=10001$.

We wish to identify groups of genes that tend to be grouped together in multiple datasets. We focus upon the genes that tend to have the same cluster label in multiple datasets, those which have a common label across some set of datasets in more than half of the observed clusterings, or $\hat{P}\left(c_{n l}=c_{n m}\right)>0.5$, where $c_{n l}$ denotes the cluster label of gene $n$ in dataset $l$. This based upon the the concept of fused genes proposed by Savage et al. (2010) and used by Kirk et al. (2012), but to avoid confusion due to other possible ideas of fused genes (e.g. those that contribute to a common protein complex, the behaviour of TFs upon a gene) we avoid this term. These genes with common clustering across datasets are those most affected by the integrative aspect of the analysis and therefore we focus upon these in the our cluster analysis. In our case we have the possible sets of:

- $\{$ Time course $\},\{$ ChIP-chip $\},\{$ PPI $\}$,

- $\{$ Time course, ChIP-chip $\},\{$ Time course, PPI $\},\{$ ChIP-chip, PPI $\}$, and

- $\{$ Time course, ChIP-chip, PPI $\}$.

The number of genes meeting this criteria between any two datasets is indicative of how strongly they influence each other and is expected to align with the $\phi_{l m}$ parameters from the MDI model. We find the following number of unique genes integrated between each combination of datasets:

- Time course + ChIP-chip + PPI: 56, 
bioRxiv preprint doi: $h t t p s: / / d o i . o r g / 10.1101 / 2020.1217 .423244^{-}$this version posted March 13,2021 . The copyright holder for this preprint (which was not certified by peer review) is the author/funder, who has granted bioRxiv a license to display the preprint in perpetuity. It is made available under aCC-BY 4.0 International license.

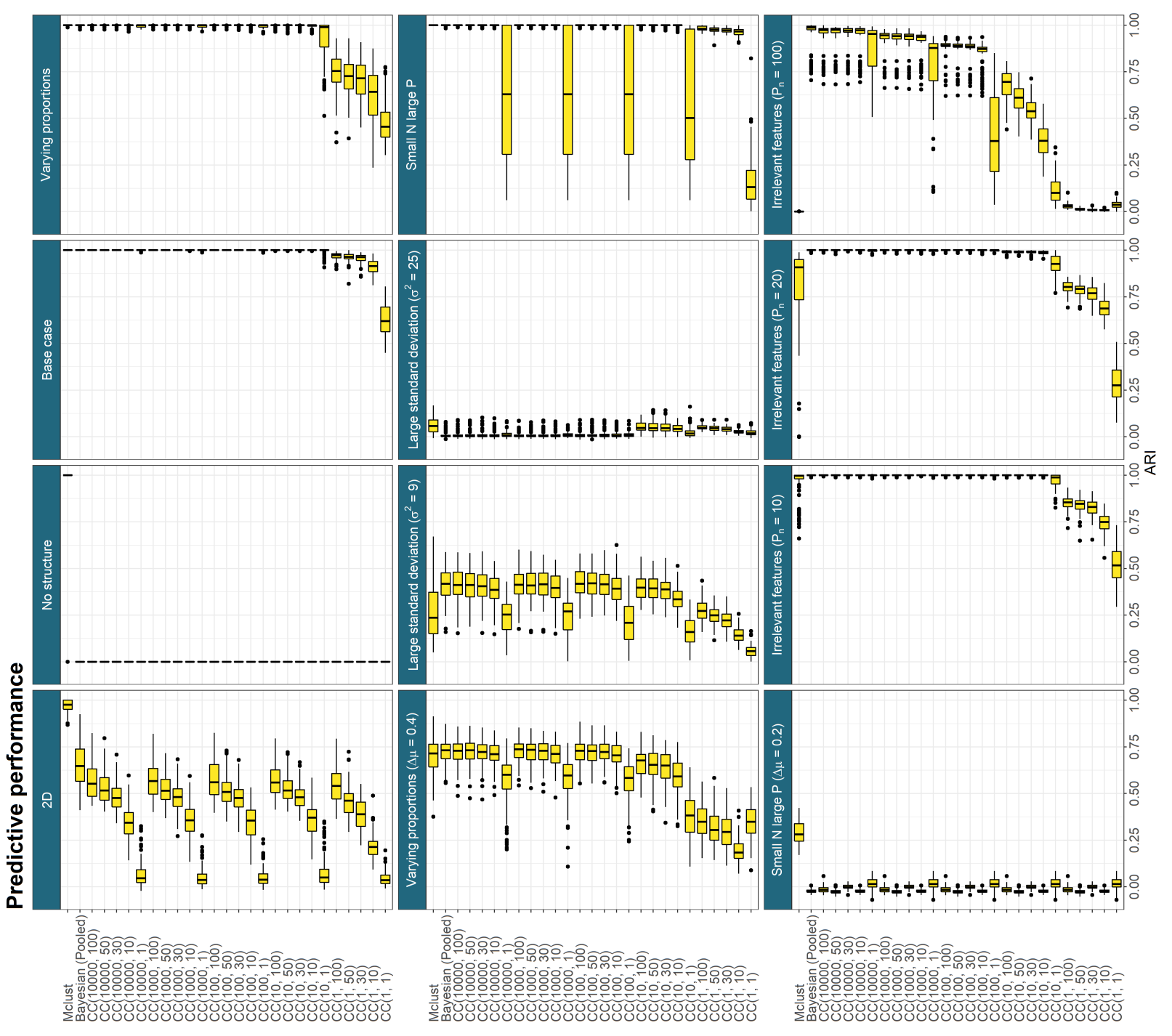

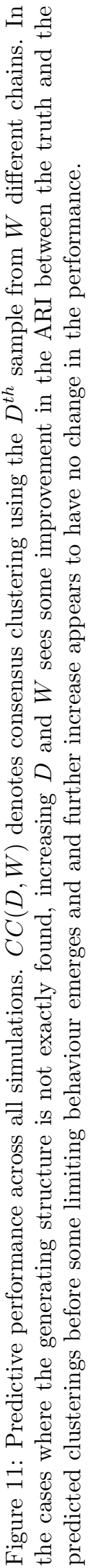


Time course PPI

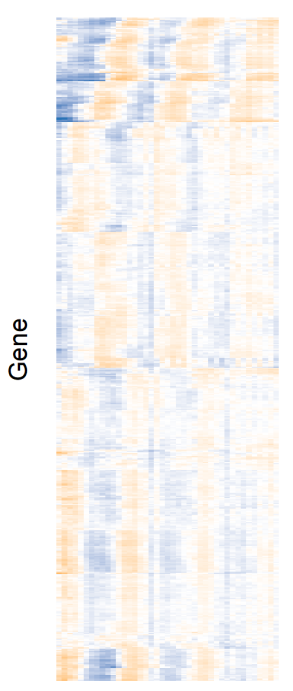

Time point

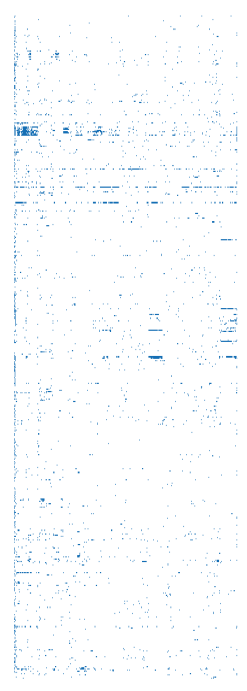

Protein

\section{ChIP-chip}

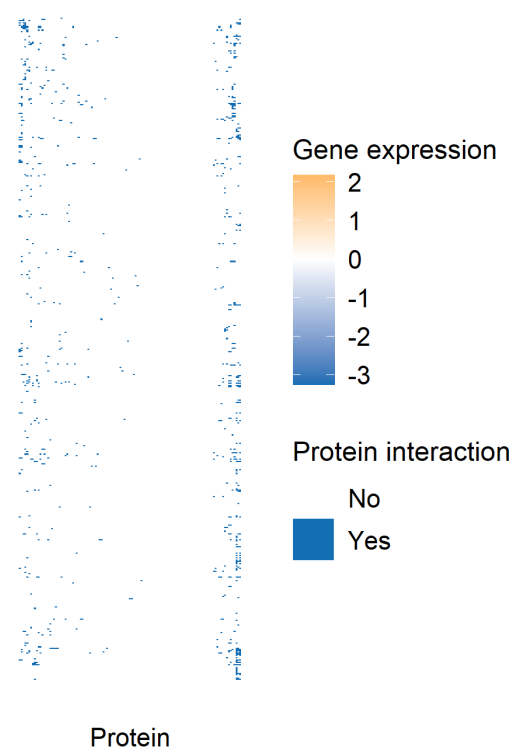

Figure 12: Heatmap of the yeast datasets. Each plot has a common row order corresponding to the gene products being clustered. This order was decided by a hierarchical clustering of the rows of the time course expression matrix. The time course data is associated with the "Gene expression" legend and the ChIP-chip and PPI data with "Protein interaction" legend.

- Time course + ChIP-chip: 205 (261 including the 56 integrated across all datasets),

- Time course + PPI: 12 (68),

- ChIP-chip + PPI: 43 (99).

This shows that the time course and ChIP-chip datasets contain very similar structure, the ChIP-chip and PPI datasets have some similarity but significantly less and the time course and PPI datasets have less shared signal again.

Compare this to the original analysis of this data in Kirk et al. (2012), where the number of such genes in each combination is:

- Time course + ChIP-chip + PPI: 16,

- Time course + ChIP-chip: 32 (48),

- Time course + PPI: 16 (32),

- ChIP-chip + PPI: 15 (31).

Our analysis has found significantly more shared structure.

\subsubsection{Time course ChIP-chip analysis}

We focus upon the dataset pairing of time course + ChIP-chip within the integrative analysis as the combination with the greatest number of genes with shared clustering as stated in the main paper. We find that a small number of transcription factors dominate the clustering in the ChIP-chip dataset (as shown in figure 6 of the main paper), with different combinations emerging across the 10 clusters shown here in table 2. Many of these 10 correspond to transcription factors that are well known to regulate cell cycle expression, namely MBP1, SWI4, SWI6, MCM1, FKH1, FKH2, NDD1, SWI5, and ACE2 (Simon et al., 2001). 


\section{Time course}

\section{Consensus matrices}
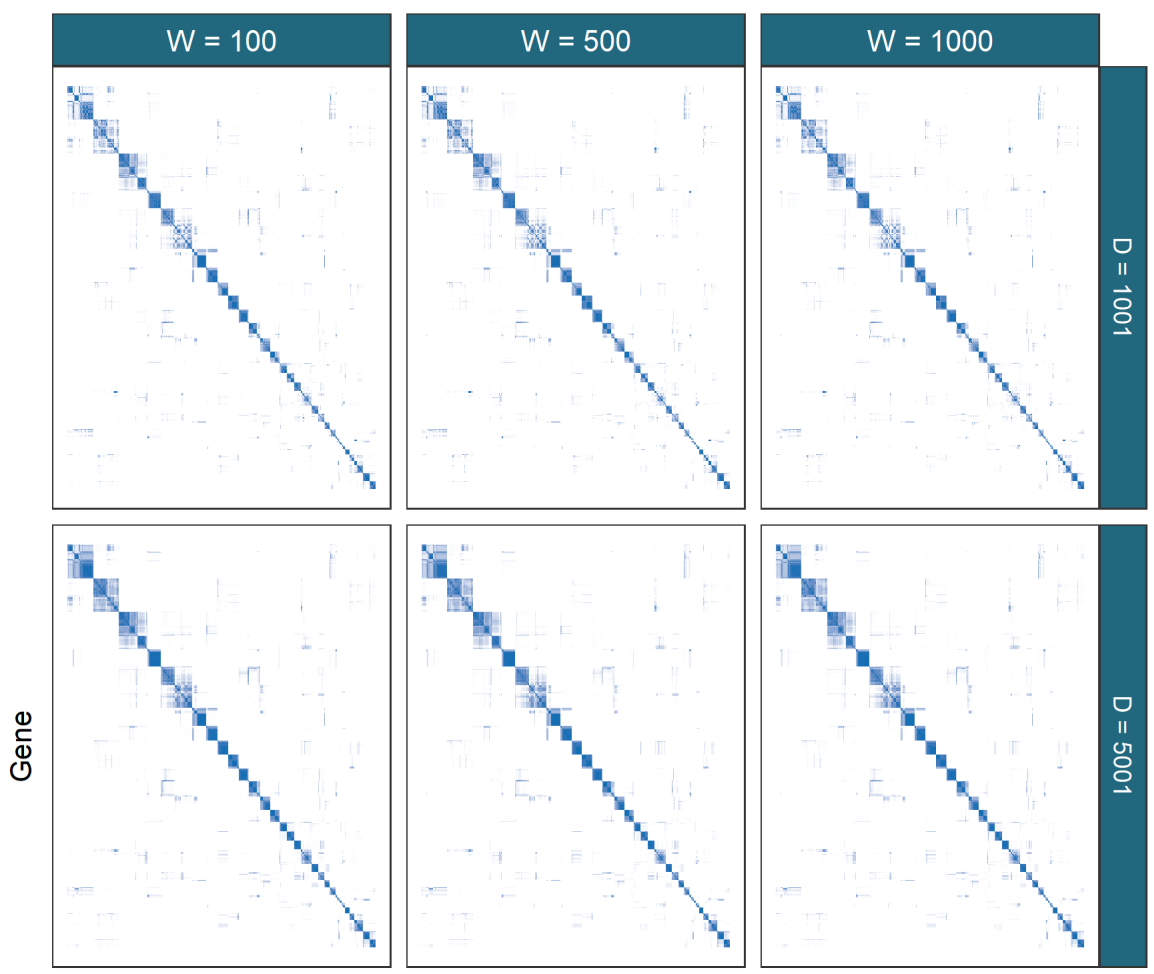

Coclustering

proportion
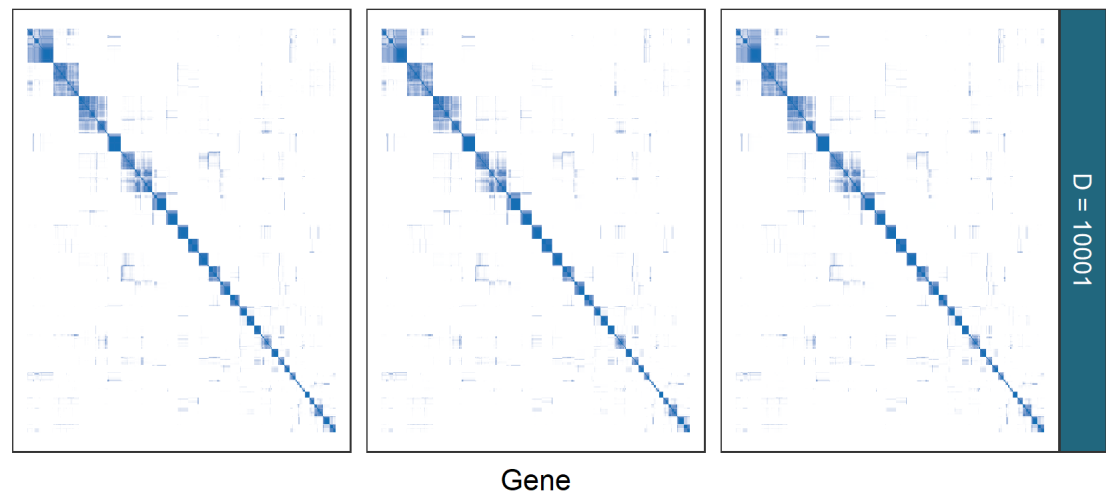

1.00

0.75

0.50

0.25

0.00

Figure 13: Consensus matrices for different ensembles of MDI for the time course data. This dataset has stable clustering across the different choices of number of chains, $W$, and chain depth, $D$, with some components merging as the chain depth increases. 


\section{ChIP-chip}

\section{Consensus matrices}
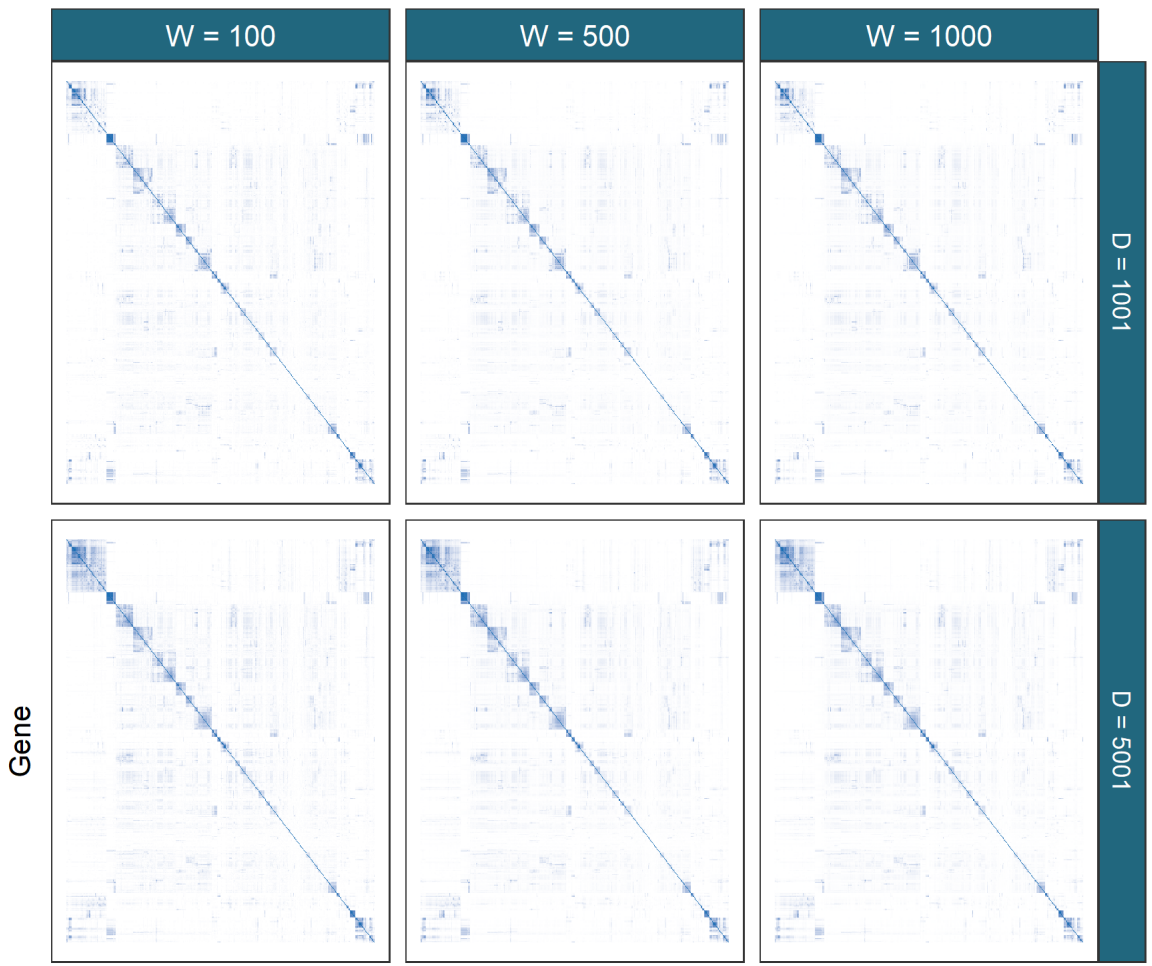

Coclustering

proportion
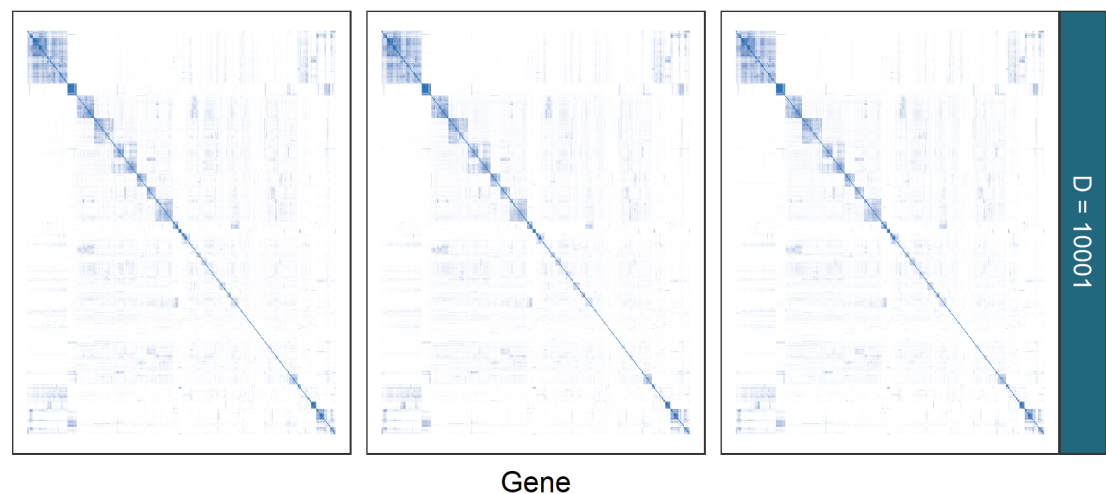

0.50

0.25

0.00

Figure 14: The ChIP-chip dataset is more sparse than the time course data. In keeping with the results from the simulations for mixture models, deeper chains are required for better performance. It is only between $D=5,001$ and $D=10,001$ that no change in the clustering can be observed and the result is believed to be stable. In this dataset the number of chains used, $W$, appears relatively unimportant, with similar results for $W=100,500,1000$. 


\section{PPI}

\section{Consensus matrices}
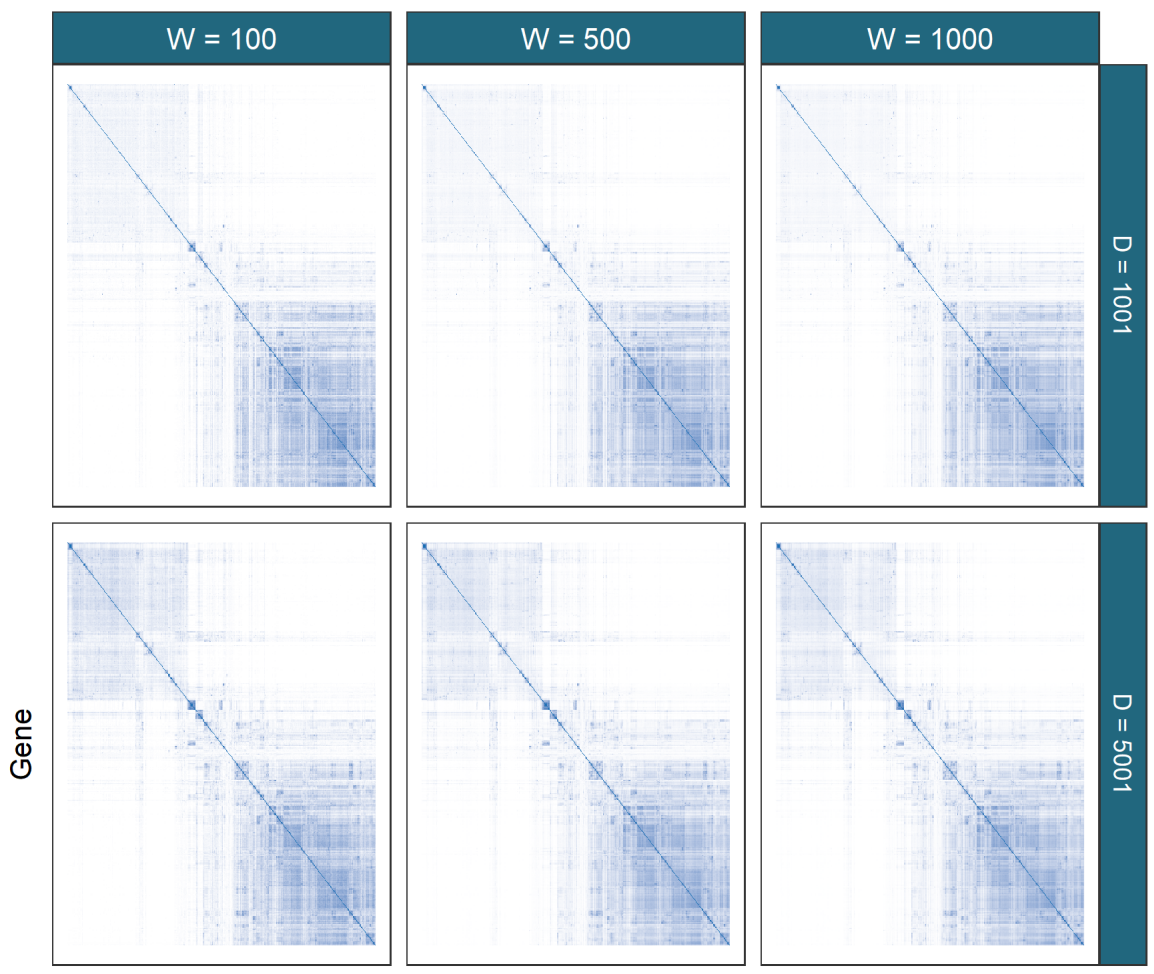

Coclustering

proportion
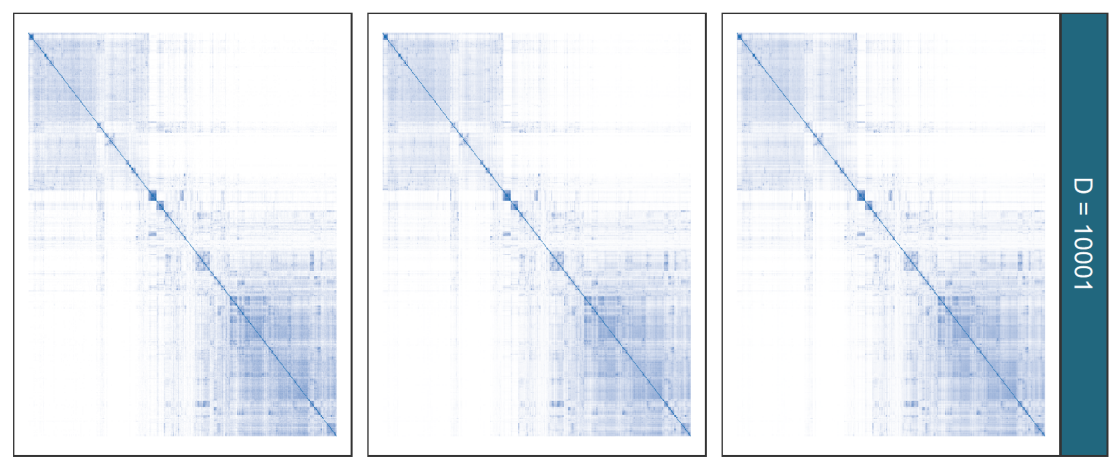

Gene

Figure 15: The PPI dataset has awkward characteristics for modelling. A wide, sparse dataset it is chain depth that we found to be the most important parameter for the ensemble. Similar to the results in figure 14 , the matrices only stabilise from $D=5001$ to $D=10001$. 
Table 2: Table of transcription factors prominent in clusters of genes with shared labels for a majority of samples for the time course and ChIP-chip datasets.

\begin{tabular}{|c|c|c|}
\hline Gene & Name & Description \\
\hline YLR131C & ACE2 & $\begin{array}{l}\text { Transcription factor required for septum destruction af- } \\
\text { ter cytokinesis; phosphorylation by Cbk1p blocks nu- } \\
\text { clear exit during M/G1 transition; phosphorylation by } \\
\text { cyclins Cdc28p and Pho85p prevents nuclear import } \\
\text { during cell cycle phases other than cytokinesis; part of } \\
\text { RAM network that regulates cellular polarity and mor- } \\
\text { phogenesis; ACE2 has a paralog, SWI5, that arose from } \\
\text { the whole genome duplication }\end{array}$ \\
\hline YPL049C & DIG1 & $\begin{array}{l}\text { MAP kinase-responsive inhibitor of the Ste12p tran- } \\
\text { scription factor; involved in the regulation of mating- } \\
\text { specific genes and the invasive growth pathway; Dig } 1 p \\
\text { and paralog Dig } 2 p \text { bind to Ste12p }\end{array}$ \\
\hline YIL131C & FKH1 & $\begin{array}{l}\text { Forkhead family transcription factor; evolutionarily con- } \\
\text { served lifespan regulator; binds multiple chromosomal } \\
\text { elements with distinct specificities, cell cycle dynamics; } \\
\text { regulates transcription elongation, chromatin silencing } \\
\text { at mating loci, expression of } \mathrm{G} 2 / \mathrm{M} \text { phase genes }\end{array}$ \\
\hline YNL068C & FKH2 & $\begin{array}{l}\text { Forkhead family transcription factor; rate-limiting ac- } \\
\text { tivator of replication origins; evolutionarily conserved } \\
\text { regulator of lifespan; binds multiple chromosomal el- } \\
\text { ements with distinct specificities, cell cycle dynamics; } \\
\text { positively regulates transcriptional elongation; negative } \\
\text { role in chromatin silencing at HML and HMR; major } \\
\text { role in expression of G2/M phase genes }\end{array}$ \\
\hline YDL056W & MBP1 & $\begin{array}{l}\text { Transcription factor; involved in regulation of cell cy- } \\
\text { cle progression from G1 to } \mathrm{S} \text { phase, forms a complex } \\
\text { with Swi6p that binds to MluI cell cycle box regulatory } \\
\text { element in promoters of DNA synthesis genes }\end{array}$ \\
\hline YMR043W & MCM1 & $\begin{array}{l}\text { Transcription factor; involved in cell-type-specific tran- } \\
\text { scription and pheromone response; plays a central role in } \\
\text { the formation of both repressor and activator complexes; } \\
\text { involved in the transcription of some M/G1 genes Simon } \\
\text { et al. (2001). }\end{array}$ \\
\hline YOR372C & NDD1 & $\begin{array}{l}\text { Transcriptional activator essential for nuclear division; } \\
\text { essential component of the mechanism that activates } \\
\text { the expression of a set of late-S-phase-specific genes; } \\
\text { turnover is tightly regulated during cell cycle and in re- } \\
\text { sponse to DNA damage }\end{array}$ \\
\hline YHR084W & STE12 & $\begin{array}{l}\text { Transcription factor that is activated by a MAPK signal- } \\
\text { ing cascade; activates genes involved in mating or pseu- } \\
\text { dohyphal/invasive growth pathways; cooperates with } \\
\text { Tec1p transcription factor to regulate genes specific for } \\
\text { invasive growth }\end{array}$ \\
\hline YER111C & SWI4 & $\begin{array}{l}\text { DNA binding component of the SBF complex (Swi4p- } \\
\text { Swi6p); a transcriptional activator that in concert with } \\
\text { MBF (Mbp1-Swi6p) regulates late G1-specific transcrip- } \\
\text { tion of targets including cyclins and genes required for } \\
\text { DNA synthesis and repair }\end{array}$ \\
\hline
\end{tabular}


YDR146C SWI5 Transcription factor that recruits Mediator and Swi/Snf complexes; activates transcription of genes expressed at the M/G1 phase boundary and in G1 phase; required for expression of the HO gene controlling mating type switching; localization to nucleus occurs during G1 and appears to be regulated by phosphorylation by Cdc28p kinase; SWI5 has a paralog, ACE2, that arose from the whole genome duplication

YLR182W SWI6 Transcription cofactor; forms complexes with Swi4p (SBF) and Mbp1p (MBF) to regulate transcription at the G1/S transition (Simon et al., 2001); involved in meiotic gene expression; also binds Stb1p to regulate transcription at START; also required for the unfolded protein response, independently of its known transcriptional coactivators

YBR083W TEC1 Transcription factor targeting filamentation genes and Ty1 expression; Ste12p activation of most filamentation gene promoters depends on Tec1p and Tec1p transcriptional activity is dependent on its association with Ste12p; binds to TCS elements upstream of filamentation genes, which are regulated by Tec1p/Ste12p/Dig1p complex; competes with Dig2p for binding to Ste12p/Dig1p; positive regulator of chronological life span

YML027W YOX1 Homeobox transcriptional repressor; binds to Mcm1p and to early cell cycle boxes (ECBs) in the promoters of cell cycle-regulated genes expressed in M/G1 phase; phosphorylated by the cyclin Cdc28p; relocalizes from nucleus to cytoplasm upon DNA replication stress

These regulatory proteins are found in different combinations across the clusters. Based upon these combinations we associate each cluster with phases of the cell cycle and or some specific processes.

- Cluster 1: both ACE2 and SWI5 emerge. These regulate specific genes at the end of M and early G1 (McBride et al., 1999; Simon et al., 2001).

- Cluster 2: SWI5. This is similar to cluster 1, as ACE2 is a paralog of SWI5; therefore associated with M/G1. Furthermore, inspection of the expression in the timecourse data shows that the members of cluster 2 largely differentiate from those of cluster 1 based upon amplitude, not periodicity, suggesting that these clusters could be merged.

- Cluster 5: MBP1, SWI4 and SWI6. The SBF complex (Swi4p-Swi6p) is a transcriptional activator that in concert with MBF (Mbp1-Swi6p) regulates late G1-specific transcription of targets including cyclins and genes required for DNA synthesis and repair, controlling the transition to S phase (Simon et al., 2001; Iyer et al., 2001; Aligianni et al., 2009).

- Cluster 9: MBP1 and SWI6. These combine to form MBF, which regulates DNA replication and repair (Iyer et al., 2001).

- Cluster 11: DIG1, SWI4, SWI6, and STE12 emerge in all members with some having associations with TEC1. TEC1 and STE12, controls development, including cell adhesion and filament formation and is negatively regulated by DIG1 and DIG2 (van der Felden et al., 2014).

- Cluster 12: MBP1, SWI4 and SWI6. Similar to cluster 5 in both the time course and ChIP-chip datasets and thus G1/S phase. 
- Cluster 16: some MBP1, SWI4 and SWI6. The constituents of this cluster are largely associated with proteins contributing to histones H1, H2A, H2B, H3 and H4, suggesting an S-phase cluster (Ewen, 2000).

- Cluster 17: FKH1 and FKH2. Fkh1p and Fkh2p are required for cell-cycle regulation of transcription during G2/M (Kumar et al., 2000).

- Cluster 20: NDD1 and MCM1 with some FKH2. Mcm1, together with Fkh1 or Fkh2, recruits the Ndd1 protein in late G2, and thus controls the transcription of G2/M genes (Simon et al., 2001; Koranda et al., 2000).

- Cluster 26: YOX1 and MCM1. YOX1 binds to Mcm1p and to early cell cycle boxes (ECBs) in the promoters of cell cycle-regulated genes expressed in M/G1 phase (Pramila et al., 2002).

\begin{tabular}{|c|c|c|c|}
\hline Gene & Name & Cluster & Description \\
\hline YJL115W & ASF1 & 9 & $\begin{array}{l}\text { Nucleosome assembly factor; involved in chromatin as- } \\
\text { sembly, disassembly; required for buffering mRNA syn- } \\
\text { thesis rate against gene dosage changes in S phase }\end{array}$ \\
\hline YLR103C & CDC45 & 9 & $\begin{array}{l}\text { DNA replication initiation factor; recruited to MCM } \\
\text { pre-RC complexes at replication origins; recruits elonga- } \\
\text { tion machinery; binds tightly to ssDNA, which disrupts } \\
\text { interaction with the MCM helicase and stalls it during } \\
\text { replication stress; mutants in human homolog may cause } \\
\text { velocardiofacial and DiGeorge syndromes }\end{array}$ \\
\hline YPL241C & CIN2 & 9 & $\begin{array}{l}\text { GTPase-activating protein (GAP) for Cin4p; tubulin } \\
\text { folding factor C involved in beta-tubulin (Tub2p) fold- } \\
\text { ing; mutants display increased chromosome loss and } \\
\text { benomyl sensitivity; human homolog RP2 complements } \\
\text { yeast null mutant }\end{array}$ \\
\hline YPR175W & DPB2 & 9 & $\begin{array}{l}\text { Second largest subunit of DNA polymerase II (DNA } \\
\text { polymerase epsilon); required for maintenance of fi- } \\
\text { delity of chromosomal replication; essential motif in C- } \\
\text { terminus is required for formation of the four-subunit } \\
\text { Pol epsilon; expression peaks at the G1/S phase bound- } \\
\text { ary; Cdc } 28 \text { p substrate }\end{array}$ \\
\hline YIL026C & IRR1 & 9 & $\begin{array}{l}\text { Subunit of the cohesin complex; which is required for sis- } \\
\text { ter chromatid cohesion during mitosis and meiosis and } \\
\text { interacts with centromeres and chromosome arms }\end{array}$ \\
\hline YCL061C & MRC1 & 9 & $\begin{array}{l}\text { S-phase checkpoint protein required for DNA replica- } \\
\text { tion; couples DNA helicase and polymerase; defines a } \\
\text { novel S-phase checkpoint with Hog1p that coordinates } \\
\text { DNA replication and transcription upon osmostress; } \\
\text { protects uncapped telomeres; Dia2p-dependent degra- } \\
\text { dation mediates checkpoint recovery; mammalian } \\
\text { claspin homolog; subunit of a replication-pausing } \\
\text { checkpoint complex, Tof1p-Mrc1p-Csm3p; checkpoint- } \\
\text { mediator protein that functions during DNA replication } \\
\text { and activates the effector kinase Rad53 (Bando et al., } \\
\text { 2009); human ATR homolog (Lao et al., 2018) }\end{array}$ \\
\hline YDR097C & MSH6 & 9 & $\begin{array}{l}\text { Protein required for mismatch repair in mitosis and } \\
\text { meiosis; forms a complex with Msh2p to repair both } \\
\text { single-base and insertion-deletion mispairs; also involved } \\
\text { in interstrand cross-link repair; potentially phosphory- } \\
\text { lated by Cdc } 28 \mathrm{p}\end{array}$ \\
\hline
\end{tabular}


YNL102W POL1 9 Catalytic subunit of the DNA polymerase I alphaprimase complex; required for the initiation of DNA replication during mitotic DNA synthesis and premeiotic DNA synthesis

YBL035C POL12 $\quad 9 \quad$ B subunit of DNA polymerase alpha-primase complex; required for initiation of DNA replication during mitotic and premeiotic DNA synthesis; also functions in telomere capping and length regulation

YKL113C RAD27 $\quad 9 \quad$ 5' to 3' exonuclease, 5' flap endonuclease; required for Okazaki fragment processing and maturation, for longpatch base-excision repair and large loop repair (LLR), ribonucleotide excision repair

YPL153C RAD53 9 DNA damage response protein kinase; required for cellcycle arrest, regulation of copper genes in response to DNA damage; human homolog CHEK2 implicated in breast cancer can complement yeast null mutant

YAR007C RFA1 9 Subunit of heterotrimeric Replication Protein A (RPA); RPA is a highly conserved single-stranded DNA binding protein involved in DNA replication, repair, and recombination; RPA protects against inappropriate telomere recombination, and upon telomere uncapping, prevents cell proliferation by a checkpoint-independent pathway; role in DNA catenation/decatenation pathway of chromosome disentangling; relocalizes to the cytosol in response to hypoxia

YNL312W RFA2 $\quad 9 \quad$ Subunit of heterotrimeric Replication Protein A (RPA); RPA is a highly conserved single-stranded DNA binding protein involved in DNA replication, repair, and recombination; RPA protects against inappropriate telomere recombination, and upon telomere uncapping, prevents cell proliferation by a checkpoint-independent pathway; in concert with Sgs1p-Top2p-Rmi1p, stimulates DNA catenation/decatenation activity of Top3p; protein abundance increases in response to DNA replication $\mathrm{s}$

YAR008W SEN34 9 Subunit of the tRNA splicing endonuclease; tRNA splicing endonuclease (Sen complex) is composed of Sen2p, Sen15p, Sen34p, and Sen54p; Sen complex also cleaves the CBP1 mRNA at the mitochondrial surface; Sen34p contains the active site for tRNA 3' splice site cleavage and has similarity to Sen2p and to Archaeal tRNA splicing endonuclease

YJL074C SMC3 $\quad 9$
for sister chromatid cohesion in mitotic cells; also required, with Rec8p, for cohesion and recombination during meiosis; phylogenetically conserved SMC chromosomal ATPase family member 
YNL273W TOF1 $9 \quad$ Subunit of a replication-pausing checkpoint complex; Tof1p-Mrc1p-Csm3p acts at the stalled replication fork to promote sister chromatid cohesion after DNA damage, facilitating gap repair of damaged DNA; interacts with the MCM helicase; checkpoint-mediator protein that functions during DNA replication and activates the effector kinase RAD53 (Bando et al., 2009); human ATM homolog (Lao et al., 2018)

YMR215W GAS3 $16 \quad$ Putative 1,3-beta-glucanosyltransferase; has similarity
go other GAS family members; low abundance, possibly inactive member of the GAS family of GPI-containing proteins; localizes to the cell wall; mRNA induced during sporulation

YBR009C HHF1 $16 \quad$ Histone H4; core histone protein required for chromatin assembly and chromosome function; one of two identical histone proteins (see also HHF2); contributes to telomeric silencing; N-terminal domain involved in maintaining genomic integrity

YNL030W HHF2 $16 \quad$ Histone H4; core histone protein required for chromatin assembly and chromosome function; one of two identical histone proteins (see also HHF1); contributes to telomeric silencing; N-terminal domain involved in maintaining genomic integrity

YPL127C HHO1 16 Histone H1, linker histone with roles in meiosis and sporulation; decreasing levels early in sporulation may promote meiosis, and increasing levels during sporulation facilitate compaction of spore chromatin; binds to promoters and within genes in mature spores; may be recruited by Ume6p to promoter regions, contributing to transcriptional repression outside of meiosis; suppresses DNA repair involving homologous recombination

YBR010W HHT1 16

YNL031C HHT2 16

Histone H3; core histone protein required for chromatin assembly, part of heterochromatin-mediated telomeric and HM silencing; one of two identical histone H3 proteins (see HHT2); regulated by acetylation, methylation, and phosphorylation; H3K14 acetylation plays an important role in the unfolding of strongly positioned nucleosomes during repair of UV damage

Histone H3; core histone protein required for chromatin assembly, part of heterochromatin-mediated telomeric and HM silencing; one of two identical histone H3 proteins (see HHT1); regulated by acetylation, methylation, and phosphorylation; H3K14 acetylation plays an important role in the unfolding of strongly positioned nucleosomes during repair of UV damage

YDR225W HTA1 16

Histone $\mathrm{H} 2 \mathrm{~A}$; core histone protein required for chromatin assembly and chromosome function; one of two nearly identical subtypes (see also HTA2); DNA damage-dependent phosphorylation by Mec1p facilitates DNA repair; acetylated by Nat4p; N-terminally propionylated in vivo 


\begin{tabular}{|c|c|c|c|}
\hline YBL003C & HTA2 & 16 & $\begin{array}{l}\text { Histone } \mathrm{H} 2 \mathrm{~A} \text {; core histone protein required for chro- } \\
\text { matin assembly and chromosome function; one of } \\
\text { two nearly identical (see also HTA1) subtypes; DNA } \\
\text { damage-dependent phosphorylation by Mec1p facil- } \\
\text { tates DNA repair; acetylated by Nat4p }\end{array}$ \\
\hline YDR224C & HTB1 & 16 & $\begin{array}{l}\text { Histone H2B; core histone protein required for chro- } \\
\text { matin assembly and chromosome function; nearly identi- } \\
\text { cal to HTB2; Rad6p-Bre1p-Lge1p mediated ubiquitina- } \\
\text { tion regulates reassembly after DNA replication, tran- } \\
\text { scriptional activation, meiotic DSB formation and H3 } \\
\text { methylation }\end{array}$ \\
\hline YBL002W & HTB2 & 16 & $\begin{array}{l}\text { Histone H2B; core histone protein required for chro- } \\
\text { matin assembly and chromosome function; nearly identi- } \\
\text { cal to HTB1; Rad6p-Bre1p-Lge1p mediated ubiquitina- } \\
\text { tion regulates reassembly after DNA replication, tran- } \\
\text { scriptional activation, meiotic DSB formation and H3 } \\
\text { methylation }\end{array}$ \\
\hline YNR009W & NRM1 & 16 & $\begin{array}{l}\text { Transcriptional co-repressor of MBF-regulated gene ex- } \\
\text { pression; Nrm1p associates stably with promoters via } \\
\text { MCB binding factor (MBF) to repress transcription } \\
\text { upon exit from G1 phase }\end{array}$ \\
\hline YDR113C & PDS1 & 16 & $\begin{array}{l}\text { Securin; inhibits anaphase by binding separin Esp1p; } \\
\text { blocks cyclin destruction and mitotic exit, essential for } \\
\text { meiotic progression and mitotic cell cycle arrest; local- } \\
\text { ization is cell-cycle dependent and regulated by Cdc } 28 p \\
\text { phosphorylation }\end{array}$ \\
\hline
\end{tabular}

Table 3: Description of the genes with common labelling across the time course and ChIP-chip datasets from clusters 9 and 16.

\subsection{Bayesian analysis}

We wished to compare our results from consensus clustering to a conventional Bayesian approach. We ran 10 chains of MDI for 36 hours saving every thousandth sample. This resulted in chains of varying length. We reduced the chains to 666 samples as this was the number of samples achieved by the shortest chain. Similar to section 4.3 these chains were then investigated for

- within-chain stationarity using the Geweke convergence diagnostic (Geweke et al., 1991), and

- across-chain convergence using $\hat{R}$ (Gelman et al., 1992) and the Vats-Knudson extension (stable $\hat{R}$, Vats and Knudson, 2018).

Again we focus upon stationarity of the continuous variables. In the implementation of MDI we used, the recorded continuous variables are the concentration parameters of the Dirichlet distribution for the datasetspecific component weights and the $\phi_{i j}$ parameter associated with the correlation between the $i^{t h}$ and $j^{t h}$ datasets.

We plot the Geweke-statistic for each chain in figure 16. No chain is perfectly behaved; as we cannot reduce to the set of stationary chains we thus exclude the most poorly behaved chains. Our lack of belief in the convergence of these chains is fortified by the behaviour of $\hat{R}$ (which can be seen in figure 17) and the different distributions sampled for the $\phi_{l m}$ parameters shown in figure ??.

We visualise the the PSMs for each dataset in figure 18. 


\section{Within chain convergence}

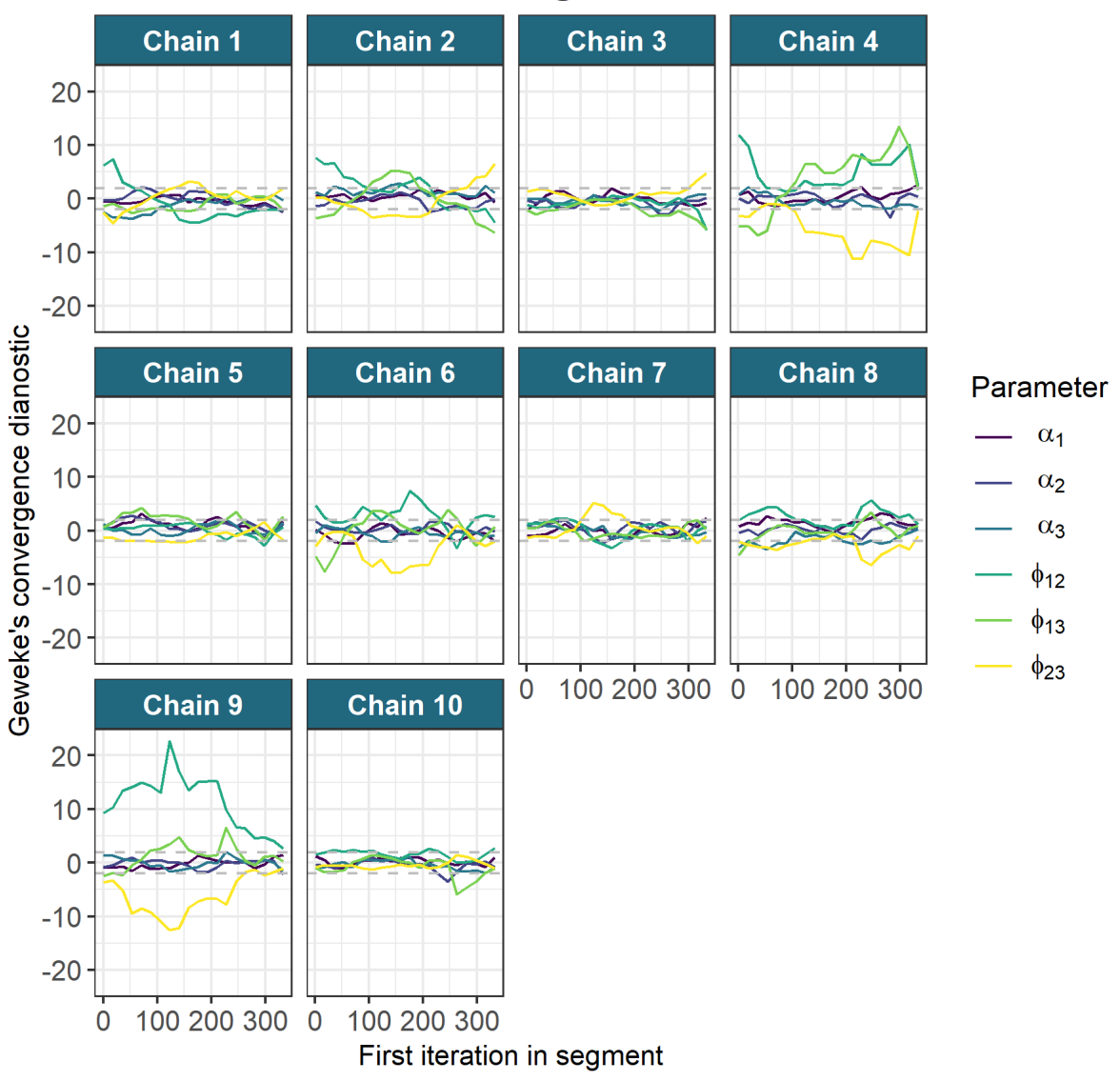

Figure 16: Chain 9 can be seen to have the most extreme behaviour in the distribution of the Geweke diagnostic for the parameters. We remove this chain from the analysis. Of the remaining chains we believe that 1,2, 4 and 6 express the distributions furthest removed from the desired behaviour and are dropped from the analysis. 


\section{Gelman-Rubin diagnostic plot}

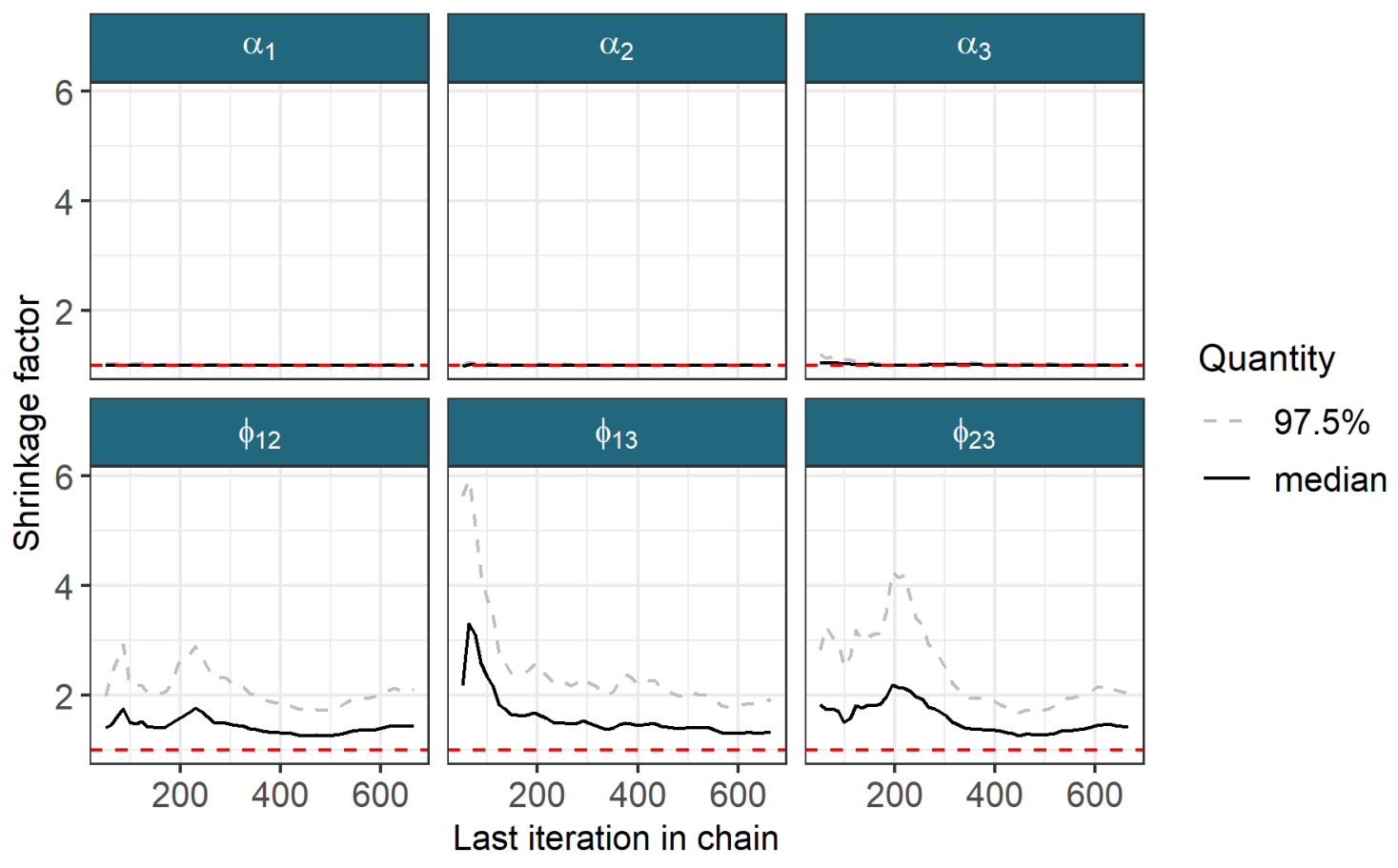

Figure 17: The chains still appear to be unconverged with $\hat{R}$ remaining above 1.25 for the $\phi_{12}, \phi_{13}$ and $\phi_{23}$ parameters. Stable $\hat{R}$ is also too high with values of $1.049,1.052$ and 1.057 for $\phi_{12}, \phi_{13}$ and $\phi_{23}$ respectively. The values of $\alpha_{l}$ cannot be seen due to the scaling of the $y$-axis. 


\section{Multi-omics analysis}

\section{Posterior similarity matrices}

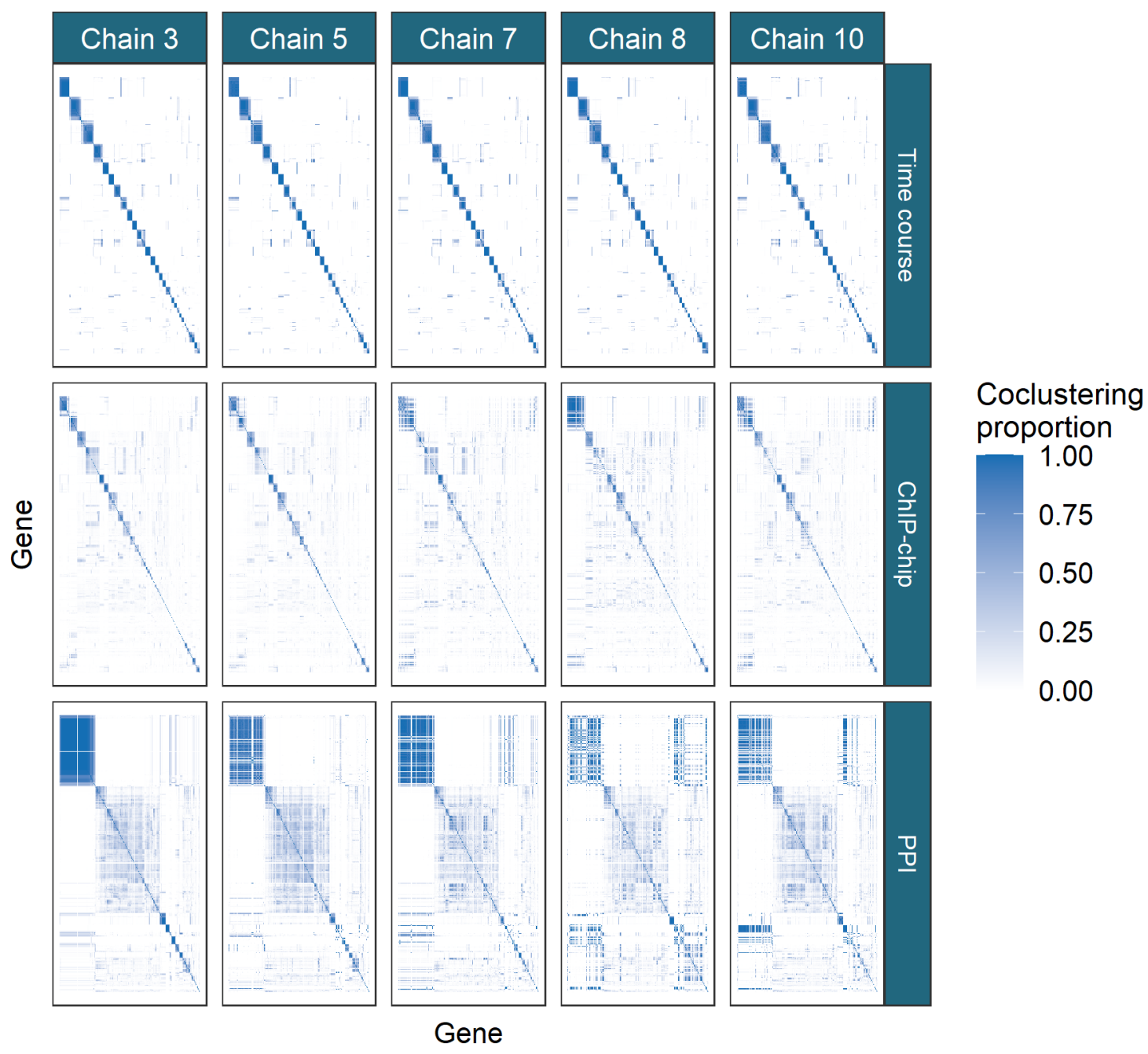

Figure 18: PSMs for each chain within each dataset. The PSMs are ordered by hierarchical clustering of the rows of the PSM for chain 3 in each dataset. There is no marked difference between the matrices for the time course data with disagreement becoming more prominent in the ChIP-chip data and more so again in the PPI dataset. 


\subsection{GO term over-representation}

We further show the lack of disagreement between the long chains from section 5.2 in a Gene Ontology (GO) term over-representation analysis. We estimated clusterings from the PSMs of the chains kept from section 5.2 visualised in figure 18 and the consensus matrix of the largest ensemble run (i.e. $C C(10001,1000)$ ) using the maxpear function from the $\mathrm{R}$ package mcclust Fritsch (2012) using default settings except for $\mathrm{k} \cdot \max$ which was set to $275 \approx N / 2$. To perform the GO term over-representation analysis we used the Bioconductor packages clusterProfiler (Yu et al., 2012), biomaRt (Durinck et al., 2009) and the annotation package org.Sc.sgd.db (Carlson et al., 2014).

We conditioned the test on the background set of the 551 yeast genes in the data. The gene labelled YIL167W was not found in the annotation database and was dropped from the analysis leaving a background universe of 550 genes. A hypergeometric test was used to check if the number of genes associated with specific GO terms within a cluster was greater than expected by random chance. We corrected the $p$-values using the Benjamini-Hochberg correction (Benjamini and Hochberg, 1995) and defined significance by a threshold of 0.01. We plotted the over-represented GO terms for the different clusterings within each dataset using the three different ontologies of "Molecular function" (MF), "Biological process" (BP) and "Cellular component" (CC) (figures 19, 20 and 21 respectively).

We find that the Bayesian chains have very significant disagreements between each other; there is no consensus on the results with many terms enriched in one or two chains. However, the consensus clustering finds many of the terms common to all of the long chains. This is what we would expect based upon the similarity of the $\phi_{l m}$ distribution in the ensemble and the pooled long chains. Consensus clustering also finds some terms with low $p$-values common to a majority of chains (such as DNA helicase activity in the MF ontology for the time course dataset) and a small number of GO terms unique to itself. These terms that no long chain find are normally related to other terms already over-represented within either the consensus clustering or a number of the long chains. For example, the transmembrane transporter activity and transporter activity terms uncovered by the ensemble in the time course dataset are related to terms found across 3 of the chains and by consensus clustering (specifically transferase activity and phosphotransferase). 


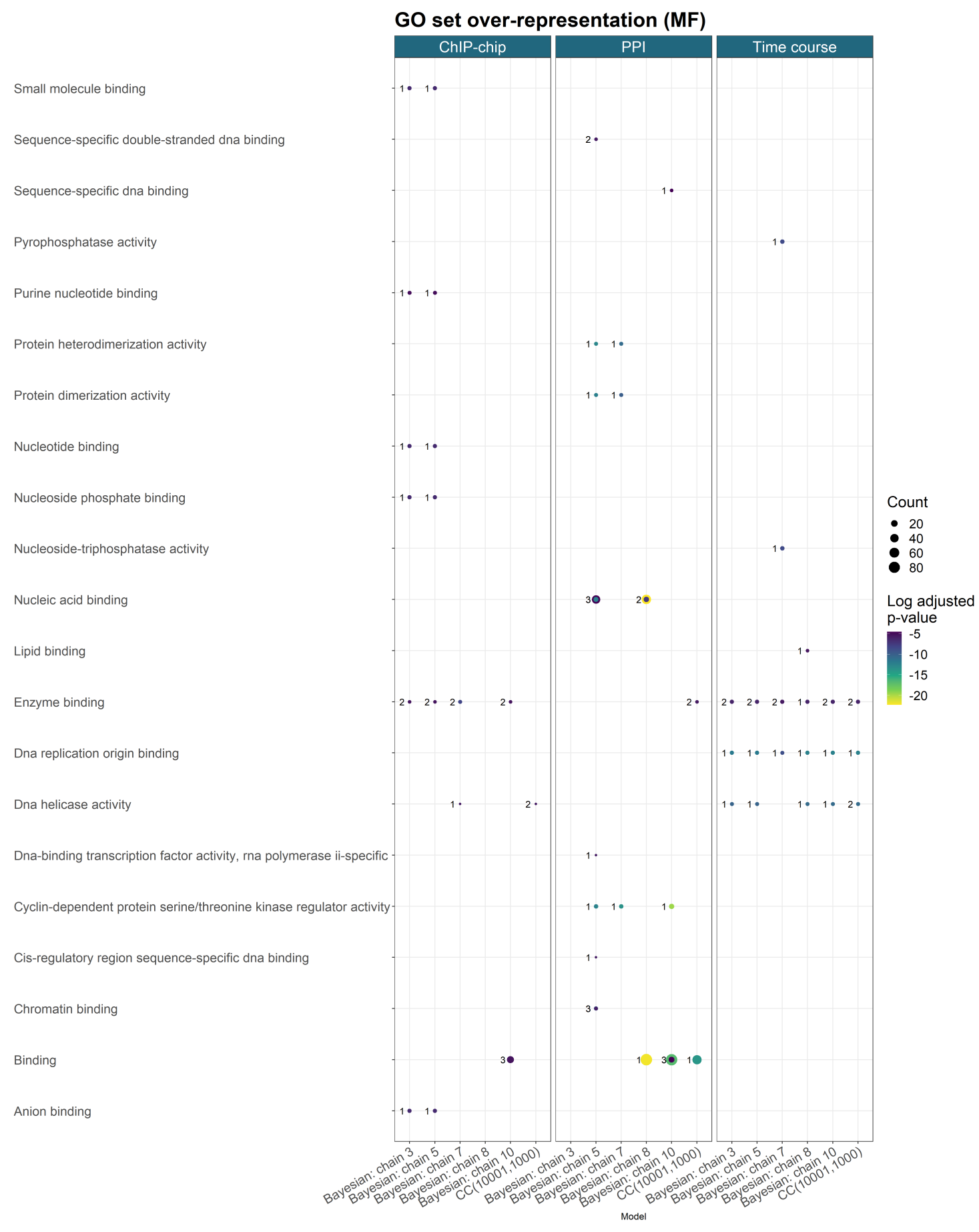

Figure 19: GO term over-representation for the Molecular function ontology for each dataset from the final clustering of each method. 
bioRxiv preprint doi: https://doi.org/10.1101/2020.12 17.423244; this version posted March 13, 2021. The copyright holder for this preprint (which was not certified by peer review) is the author/funder, who has granted bioRxiv a license to display the preprint in perpetuity. It is made available under aCC-BY 4.0 International license.

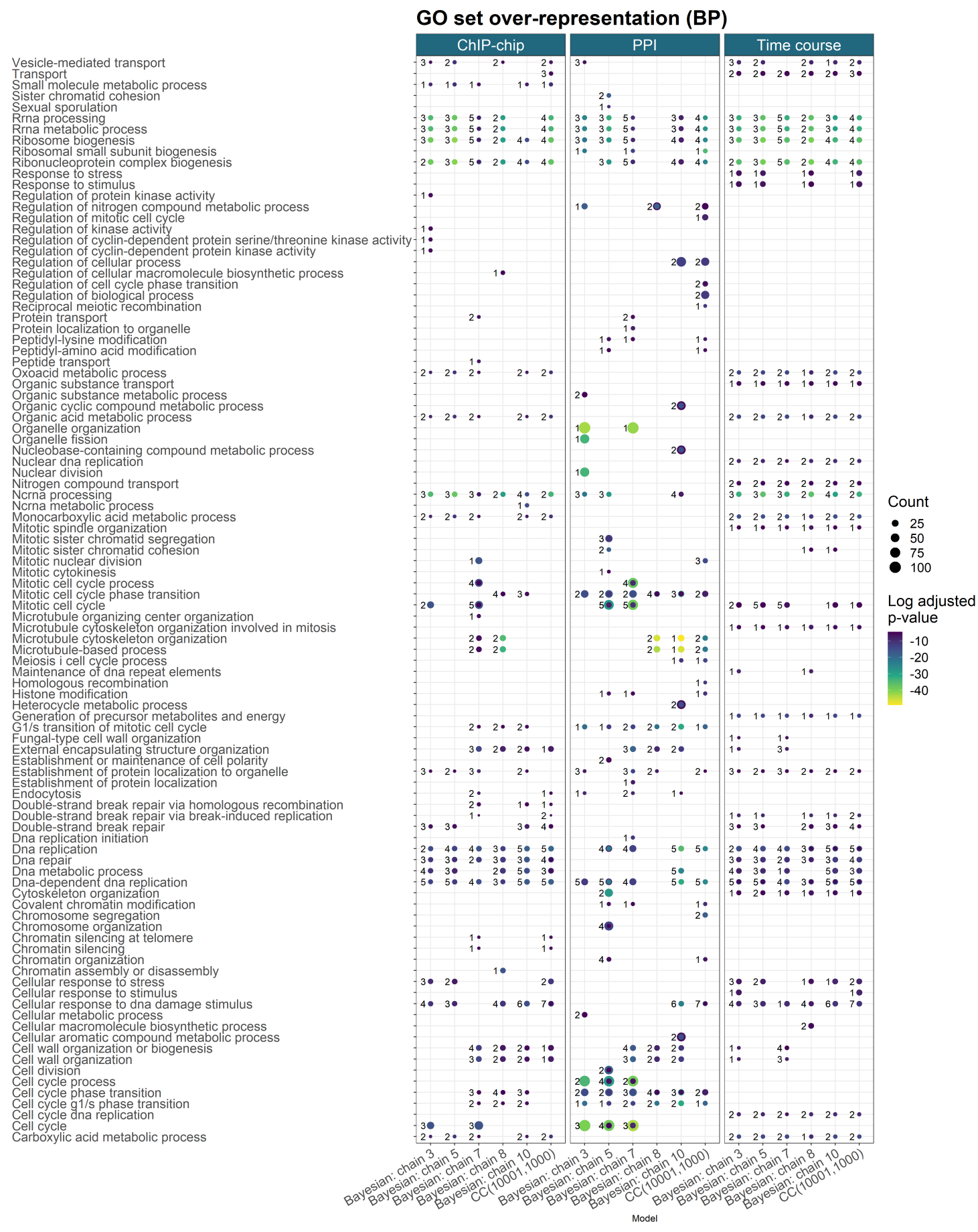

Figure 20: GO term over-representation for the Biological process ontology for each dataset from the final clustering of each method. 
bioRxiv preprint doi: https://doi.org/10.1101/2020.12.17.423244; this version posted March 13, 2021. The copyright holder for this preprint (which was not certified by peer review) is the author/funder, who has granted bioRxiv a license to display the preprint in perpetuity. It is made available under aCC-BY 4.0 International license.

Transcription regulator complex Supramolecular polymer Supramolecular fiber Supramolecular complex Storage vacuole Spindle pole body Spindle pole Spindle

Site of polarized growth Septin cytoskeleton

Ribonucleoprotein complex

Replication fork protection complex Replication fork

Protein-dna complex

Protein-containing complex

Preribosome

Polymeric cytoskeletal fibe

Plasma membrane

Organelle membran

Organelle lumen

Nucleosome

Nuclear replication fork

Nuclear outer membrane-endoplasmic reticulum membrane network

Nuclear nucleosome

Nuclear lumen

Nuclear chromosome

Nuclear chromatin

Non-membrane-bounded organelle

Microtubule organizing center

Microtubule cytoskeleton

Microtubule

Membrane protein complex

Membrane-enclosed lumen

Membrane

Lytic vacuole

Lytic vacuole

Intrinsic component of organelle membrane

Intrinsic component of membrane

Intracellular organelle lumen

Intracellular non-membrane-bounded organelle

Integral component of organelle membrane

Integral component of membrane

Incipient cellular bud site

Golgi membrane

Golgi apparatus

Fungal-type vacuole

Fungal-type cell wall

Extracellutar region

External encapsulating structure

Endoplasmic reticulum membran

Endoplasmic reticulum

ndomembrane system

Dna packaging complex

Cytosol

Cytoskeleton

Condensed nuclear chromosome kinetochore

Condensed nuclear chromosome

Condensed chromosome, centromeric region

Condensed chromosome kinetochore

Condensed chromosome

Chromosome

Chromatin

Cellular bud neck

Cellular bud

Cell wall

Cell periphery

Cell cortex

Anchored component of membrane

GO set over-representation (CC)

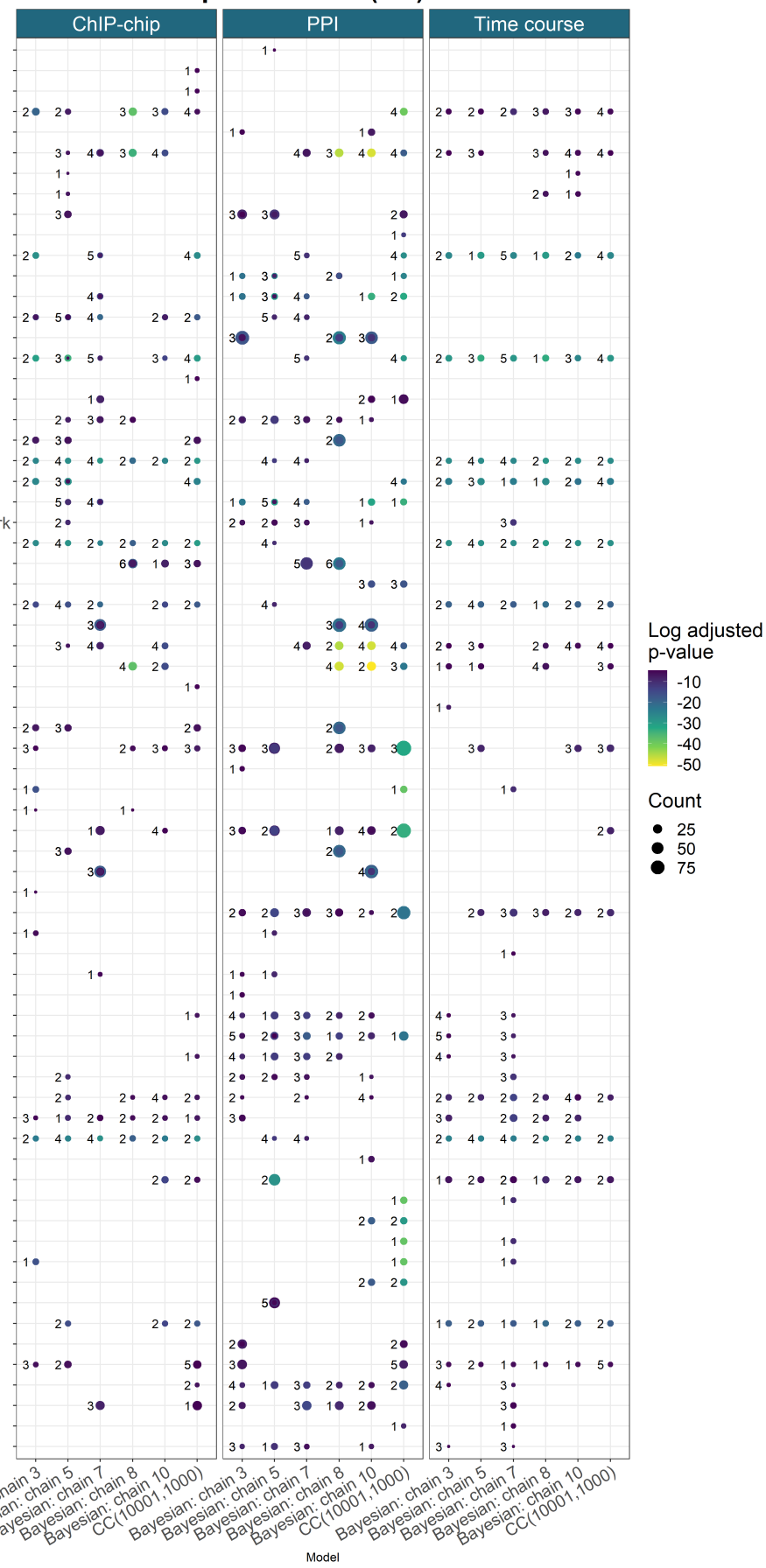

Figure 21: GO term over-representation for the Cellular component ontology for each dataset from the final clustering of each method. 


\section{References}

Sofia Aligianni, Daniel H Lackner, Steffi Klier, Gabriella Rustici, Brian T Wilhelm, Samuel Marguerat, Sandra Codlin, Alvis Brazma, Robertus AM de Bruin, and Jürg Bähler. The fission yeast homeodomain protein yox1p binds to mbf and confines mbf-dependent cell-cycle transcription to g1-s via negative feedback. PLoS Genet, 5(8):e1000626, 2009.

Masashige Bando, Yuki Katou, Makiko Komata, Hirokazu Tanaka, Takehiko Itoh, Takashi Sutani, and Katsuhiko Shirahige. Csm3, tof1, and mrc1 form a heterotrimeric mediator complex that associates with dna replication forks. Journal of Biological Chemistry, 284(49):34355-34365, 2009.

Yoav Benjamini and Yosef Hochberg. Controlling the false discovery rate: a practical and powerful approach to multiple testing. Journal of the Royal statistical society: series B (Methodological), 57(1):289-300, 1995.

M Carlson, S Falcon, H Pages, and N Li. Org. sc. sgd. db: Genome wide annotation for yeast. $R$ package version, 2(1), 2014.

Raymond J Cho, Michael J Campbell, Elizabeth A Winzeler, Lars Steinmetz, Andrew Conway, Lisa Wodicka, Tyra G Wolfsberg, Andrei E Gabrielian, David Landsman, David J Lockhart, et al. A genome-wide transcriptional analysis of the mitotic cell cycle. Molecular cell, 2(1):65-73, 1998.

Steffen Durinck, Paul T Spellman, Ewan Birney, and Wolfgang Huber. Mapping identifiers for the integration of genomic datasets with the r/bioconductor package biomart. Nature protocols, 4(8):1184, 2009.

Yasin Șenbabaoğlu, George Michailidis, and Jun Z Li. A reassessment of consensus clustering for class discovery. bioRxiv, page 002642, 2014a.

Yasin Șenbabaoğlu, George Michailidis, and Jun Z Li. Critical limitations of consensus clustering in class discovery. Scientific reports, 4(1):1-13, 2014 b.

Mark E Ewen. Where the cell cycle and histones meet. Genes \& development, 14(18):2265-2270, 2000.

Thomas S Ferguson. A bayesian analysis of some nonparametric problems. The annals of statistics, pages 209-230, 1973.

Arno Fritsch. mcclust: Process an MCMC Sample of Clusterings, 2012. URL https : //CRAN.R-project.org/package=mcclust. $R$ package version 1.0.

Arno Fritsch, Katja Ickstadt, et al. Improved criteria for clustering based on the posterior similarity matrix. Bayesian analysis, 4(2):367-391, 2009.

Evelina Gabasova, John Reid, and Lorenz Wernisch. Clusternomics: Integrative context-dependent clustering for heterogeneous datasets. PLoS computational biology, 13(10):e1005781, 2017.

Andrew Gelman, Donald B Rubin, et al. Inference from iterative simulation using multiple sequences. Statistical science, 7(4):457-472, 1992.

Andrew Gelman, John B Carlin, Hal S Stern, David B Dunson, Aki Vehtari, and Donald B Rubin. Bayesian data analysis. CRC press, 2013.

John Geweke et al. Evaluating the accuracy of sampling-based approaches to the calculation of posterior moments, volume 196. Federal Reserve Bank of Minneapolis, Research Department Minneapolis, MN, 1991.

Vishwanath R Iyer, Christine E Horak, Charles S Scafe, David Botstein, Michael Snyder, and Patrick O Brown. Genomic binding sites of the yeast cell-cycle transcription factors sbf and mbf. Nature, 409(6819): $533-538,2001$.

Paul Kirk, Jim E Griffin, Richard S Savage, Zoubin Ghahramani, and David L Wild. Bayesian correlated clustering to integrate multiple datasets. Bioinformatics, 28(24):3290-3297, 2012. 
Christina Knudson and Dootika Vats. stableGR: A Stable Gelman-Rubin Diagnostic for Markov Chain Monte Carlo, 2020. URL https://CRAN.R-project.org/package=stableGR. R package version 1.0.

Manfred Koranda, Alexander Schleiffer, Lukas Endler, and Gustav Ammerer. Forkhead-like transcription factors recruit ndd1 to the chromatin of g2/m-specific promoters. Nature, 406(6791):94-98, 2000.

Raman Kumar, David M Reynolds, Andrej Shevchenko, Anna Shevchenko, Sherilyn D Goldstone, and Stephen Dalton. Forkhead transcription factors, fkh1p and fkh2p, collaborate with mcm1p to control transcription required for m-phase. Current Biology, 10(15):896-906, 2000.

Jessica P Lao, Katie M Ulrich, Jeffrey R Johnson, Billy W Newton, Ajay A Vashisht, James A Wohlschlegel, Nevan J Krogan, and David P Toczyski. The yeast dna damage checkpoint kinase rad53 targets the exoribonuclease, xrn1. G3: Genes, Genomes, Genetics, 8(12):3931-3944, 2018.

Samuel A Mason, Faiz Sayyid, Paul DW Kirk, Colin Starr, and David L Wild. Mdi-gpu: accelerating integrative modelling for genomic-scale data using gp-gpu computing. Statistical Applications in Genetics and Molecular Biology, 15(1):83-86, 2016.

Helen J McBride, Yaxin Yu, and David J Stillman. Distinct regions of the swi5 and ace2 transcription factors are required for specific gene activation. Journal of Biological Chemistry, 274(30):21029-21036, 1999.

Stefano Monti, Pablo Tamayo, Jill Mesirov, and Todd Golub. Consensus clustering: a resampling-based method for class discovery and visualization of gene expression microarray data. Machine learning, 52 (1-2):91-118, 2003.

Tata Pramila, Shawna Miles, Debraj GuhaThakurta, Dave Jemiolo, and Linda L Breeden. Conserved homeodomain proteins interact with mads box protein $\mathrm{mcm} 1$ to restrict ecb-dependent transcription to the m/g1 phase of the cell cycle. Genes \& development, 16(23):3034-3045, 2002.

Sylvia Richardson and Peter J Green. On bayesian analysis of mixtures with an unknown number of components. Journal of the Royal Statistical Society: series B, 59(4):731-792, 1997.

Judith Rousseau and Kerrie Mengersen. Asymptotic behaviour of the posterior distribution in overfitted mixture models. Journal of the Royal Statistical Society: Series B (Statistical Methodology), 73(5):689-710, 2011.

Richard S Savage, Zoubin Ghahramani, Jim E Griffin, Bernard J De la Cruz, and David L Wild. Discovering transcriptional modules by bayesian data integration. Bioinformatics, 26(12):i158-i167, 2010.

Gideon Schwarz et al. Estimating the dimension of a model. The annals of statistics, 6(2):461-464, 1978.

Samuel Sanford Shapiro and Martin B Wilk. An analysis of variance test for normality (complete samples). Biometrika, 52(3/4):591-611, 1965.

Itamar Simon, John Barnett, Nancy Hannett, Christopher T Harbison, Nicola J Rinaldi, Thomas L Volkert, John J Wyrick, Julia Zeitlinger, David K Gifford, Tommi S Jaakkola, et al. Serial regulation of transcriptional regulators in the yeast cell cycle. Cell, 106(6):697-708, 2001.

Paul T Spellman, Gavin Sherlock, Michael Q Zhang, Vishwanath R Iyer, Kirk Anders, Michael B Eisen, Patrick O Brown, David Botstein, and Bruce Futcher. Comprehensive identification of cell cycle-regulated genes of the yeast saccharomyces cerevisiae by microarray hybridization. Molecular biology of the cell, 9 (12):3273-3297, 1998.

Julia van der Felden, Sarah Weisser, Stefan Brückner, Peter Lenz, and Hans-Ulrich Mösch. The transcription factors tec 1 and ste12 interact with coregulators msa1 and msa2 to activate adhesion and multicellular development. Molecular and cellular biology, 34(12):2283-2293, 2014.

Zoé Van Havre, Nicole White, Judith Rousseau, and Kerrie Mengersen. Overfitting bayesian mixture models with an unknown number of components. PloS one, 10(7):e0131739, 2015. 
Dootika Vats and Christina Knudson. Revisiting the gelman-rubin diagnostic. arXiv preprint arXiv:1812.09384, 2018.

Guangchuang Yu, Li-Gen Wang, Yanyan Han, and Qing-Yu He. clusterprofiler: an r package for comparing biological themes among gene clusters. OMICS: A Journal of Integrative Biology, 16(5):284-287, 2012. doi: 10.1089/omi.2011.0118. 